\begin{tabular}{|c|l|}
\hline Title & Evolving graphs by singular weighted curvature \\
\hline Author(s) & Giga, M.-H; Giga, Y. \\
\hline Citation & Hokkaido University Preprint Series in Mathematics, 331, 1-94 \\
\hline Issue Date & 1996-3-1 \\
\hline DOI & 10.14943/83477 \\
\hline Doc URL & http://hdl.handle.net/2115/69081 \\
\hline Type & bulletin (article) \\
\hline File Information & pre331.pdf \\
\hline
\end{tabular}

Instructions for use 


\section{M.-H. Giga and Y. Giga}

Series \#331. March 1996 


\section{HOKKAIDO UNIVERSITY PREPRINT SERIES IN MATHEMATICS}

\#307 J. Yoshizaki, On the structure of the singular set of a complex analytic foliation, 25 pages. 1995.

\#308 A. Arai, Representation of canonical commutation relations in a gauge theory, the Aharonov-Bohm effect, and Dirac Weyl operator, 17 pages. 1995.

\#309 A. Arai and M. Hirokawa, On the existence and uniqueness of ground states of the spin-boson Hamiltonian, 20 pages. 1995 .

\#310 S. Izumiya and T. Sano, Generic affine differential geometry of plane curves, 8 pages. 1995.

\#311 N. Kawazumi, On the stable cohomology algebra of extended mapping class groups for surfaces, 13 pages. 1995.

\#312 H.M.Ito and T. Mikami, Poissonian asymptotics of a randomly perturbed dynamical system: Flip-flop of the Stochastic Disk Dynamo, 20 pages. 1995.

\#313 T. Nakazi, Slice maps and multipliers of invariant subspaces, 11 pages. 1995.

\#314 - T. Mikami, Weak convergence on the first exit time of randomly perturbed dynamical systems with a repulsive equilibrium point, 20 pages. 1995.

\#315 A. Arai, Canonical commutation relations, the Weierstrass Zetafunction, and infinite dimensional Hilbert space representations of the quantum group $U_{q}\left(\mathfrak{s l}_{2}\right), 22$ pages. 1995.

\#316 Y. Shibukawa, Vertex-face correspondence in elliptic solutions of the Yang-Baxter equation, 8 pages. 1995.

\#317 M.-H. Giga and Y. Giga, Consistency in evolutions by crystalline curvature, 16 pages. 1995.

\#318 Wei-Zhi Sun, Shadows of moving surfaces, 19 pages. 1995.

\#319 S. Izumiya and G.T. Kossioris, Bifurcations of shock waves for viscosity solutions of Hamilton-Jacobi equations of one space variable, 39 pages. 1995.

\#320 T. Teruya, Normal intermediate subfactors, 44 pages. 1995.

\#321 M. Ohnuma, Axisymmetric solutions and singular parabolic equations in the theory of viscosity solutions, 26 pages. 1995 .

\#322 T. Nakazi, An outer function and several important functions in two variables, 12 pages. 1995.

\#323 N. Kawazumi, An infinitesimal approach to the stable cohomology of the moduli of Riemann surfaces, 22 pages. 1995.

\#324 A. Arai, Factorization of self-adjoint operators by abstract Dirac operators and its application to second quantizations on Boson Fermion Fock spaces, 15 pages. 1995.

\#325 K. Sugano, On strongly separable Frobenius extensions, 11 pages. 1995.

\#326 D. Lehmann and T. Suwa, Residues of holomorphic vector fields on singular varieties, 21 pages. 1995.

\#327 K. Tsutaya, Local regularity of non-resonant nonlinear wave equations, 23 pages. 1996.

\#328 T. Ozawa and Y. Tsutsumi, Space-time estimates for null gauge forms and nonlinear Schrödinger equations, 25 pages. 1996.

\#329 O. Ogurisu, Anticommutativity and spin 1/2 Schrödinger operators with magnetic fields, 12 pages. 1996.

\#330 Y. Kurokawa, Singularities for projections of contour lines of surfaces onto planes, 24 pages. 1996. 


\title{
EVOLVING GRAPHS BY SINGULAR WEIGHTED CURVATURE
}

\author{
Dedicated to Professor Rentaro Agemi \\ on the occasion of his 60 th birthday \\ Mi-Ho Giga and Yoshikazu Giga \\ Department of Mathematics, Hokkaido University \\ Sapporo 060, Japan
}

\begin{abstract}
A new notion of solutions is introduced to study degenerate nonlinear parabolic equations in one space dimension whose diffusion effect is so strong at particular slopes of unknowns that the equation is no longer a partial differential equation. Extending the theory of viscosity solutions comparison principle is established. For a periodic continuous initial data a unique global continuous solution (periodic in space) is constructed. The theory applies to motion of interfacial curves by crystalline energy or more generally by anisotropic interfacial energy with corners when the curves are the graphs of functions. Even if the driving force term exists, the initial value problem is solvable for general nonadmissible continuous (periodic) initial data.

\section{Contents.}

1. Introduction

2. Definition of generalized solutions

3. Main Theorems

4. Maximum Principle

5. Convolutions

6. Equivalent definitions of solutions

7. Proof of Comparison Theorems

8. Perron type Existence Theorem

9. Existence Theorem for periodic initial data
\end{abstract}




\section{\$1. Introduction.}

We are concerned with degenerate nonlinear parabolic equations (in one space dimension) whose diffusion effect is very strong at particular slopes of unknown functions. A typical example is a quasilinear equation

$$
u_{t}-a\left(u_{x}\right)\left(W^{\prime}\left(u_{x}\right)\right)_{x}=0,
$$

where $W$ is a given convex function on $\mathbf{R}$ but may not be $C^{1}$ so that its derivative $W^{\prime}$ may have jumps although $W^{\prime}$ is nondecreasing; here $a$ is a given nonnegative continuous function and $u_{t}$ and $u_{\infty}$ denote time and space derivative of $u$, respectively. We also consider more general form

$$
u_{t}-a\left(u_{x}\right)\left(\left(W^{\prime}\left(u_{x}\right)\right)_{x}-C(t)\right)=0
$$

with a given function $C$ or even fully nonlinear equation

$$
u_{t}+F\left(t, u_{x},\left(W^{\prime}\left(u_{x}\right)\right)_{x}\right)=0
$$

with a given function $F$ satisfying monotonicity or degenerate ellipticity condition

$$
F(t, p, X) \leq F(t, p, Y) \text { for } X \geq Y
$$

so that (1.3) is degenerate parabolic. At the first glance the evolution law given by these equation is unclear. Since $W^{\prime}$ may have jumps, so that $W^{\prime \prime}$ contains sum of delta type functions, the diffusion coefficient $a\left(u_{x}\right) W^{\prime \prime}\left(u_{x}\right)$ is no longer a function of $u_{\infty}$. For example if $W(p)=|p|$ then $W^{\prime \prime}(p)$ is two times delta function $\delta$. In this case (1.1) becomes

$$
u_{t}-2 a\left(u_{x}\right) \delta\left(u_{x}\right) u_{x x}=0
$$

which is, of course, not a classical partial differential equations (PDE). So far this type of equations was analysed only for a very restrictive class of piecewise linear unknown functions with piecewise linear $W$ [T1], [AG1] or only for (1.1) [FG]. Our eventual goal is a synthetic approach to analyse (1.3).

The purpose of this paper is threefold:(i)we introduce a new notion of solutions to (1.3), (where both solutions and $W$ need not to be piecewise linear); (ii) we establish comparison principle for our solutions; (iii) we prove the unique existence of global-in-time solutions for (1.3) (with (1.4)) when initial data is only continuous and periodic under a very weak regularity assumption on $F$. For this purpose we extend the theory of viscosity solutions [CIL] to our setting although our equations are not PDEs. It turns out that our extended version is suitable to study (1.3) when $W^{\prime}$ has jumps. 
Our equations $(1,1)-(1.3)$ stem from material sciences and physics as a geometric evolution law of interfacial curves bounding two-phase of materials $[\mathrm{Gu}]$, [Ch]. However, in the present work no knowledge of material sciences is assumed.

1.1. Notions of solutions. In [T1] J. Taylor proposed an evolution law for a special class of piecewise linear closed curves called admissible moved by crystalline energy. If the curve is represented as the graph of a function $u$, the governing equations in [T1] formally correspond to (1.1) with (positive) piecewise linear $W$, where $a$ is assumed to be proportional to $W$. Independently, Angenent and Gurtin [AG1] derived the same evolution laws (for curves) corresponding to (1.2) by establishing a continuum thermomechanical theory of crystal growth with no relation of $a$ and $W$ (even if $W$ may not be piecewise linear.) However, the class of curves are still restricted. We first reproduce their equation in our setting. Assume, for simplicity, that $W^{\prime}(p)$ has a jump at $\boldsymbol{p}=0$. Suppose that $u(t, \cdot)$ is constant on some closed interval $I(t)=(\alpha(t), \beta(t))$. Suppose in a neighborhood of $I(t)$ that $x$-derivative of $u$ has a definite sign in the left (and right) of $I(t)$ and that it is small so that $u_{x}(t, x)$ lies outside other jumps of $W^{\prime}$. Integrating (1.1) in a neighborhood $(\alpha(t)-\delta, \beta(t)+\delta), \delta>0$ of the interval $I(t)$ to get

$$
\begin{aligned}
\int_{\alpha-\delta}^{\beta+\delta} u_{t} d t & =\int_{\alpha-\delta}^{\beta+\delta} a\left(u_{x}\right)\left(W^{\prime}\left(u_{x}\right)\right)_{x} d x \\
& \sim a(0) \int_{\alpha-\delta}^{\beta+\delta}\left(W^{\prime}\left(u_{x}\right)\right)_{x} d x \quad(\delta: \text { small }) \\
& =a(0)\left\{W^{\prime}\left(u_{x}(\beta+\delta)\right)-W^{\prime}\left(u_{x}(\alpha-\delta)\right)\right\}
\end{aligned}
$$

We postulate that $u_{t}(t, x)$ is independent of $x$ on $I(t)$. Sending $\delta$ to zero, we now obtain

$$
u_{t}-a\left(u_{x}\right) \Lambda_{W}(u)=0 \text { for } x \in I(t)
$$

with

$$
\begin{aligned}
& \Lambda_{W}(u)=\chi \Delta / L, \\
& \Delta=W^{\prime}(+0)-W^{\prime}(-0), L=\beta(t)-\alpha(t) ; \\
& W^{\prime}(+0)=\lim _{\varepsilon \downarrow 0} W^{\prime}(\varepsilon), W^{\prime}(-0)=\lim _{\varepsilon \downarrow 0} W^{\prime}(-\varepsilon),
\end{aligned}
$$

here $\chi$ is the transition number and is defined by

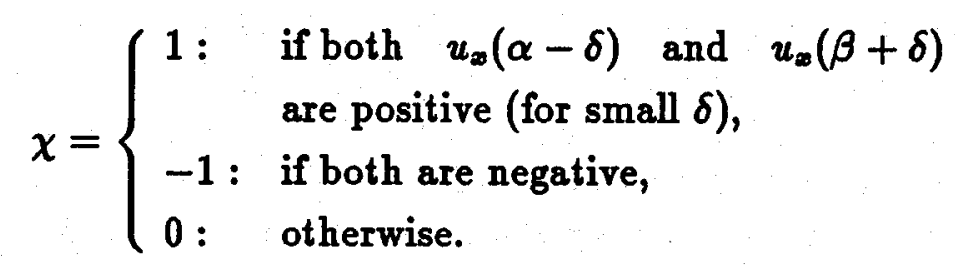


The quantity $\Lambda_{W}(u)$ is called the weighted curvature on $I(t)$. Assume that for a moment $W$ is piecewise linear and that the jumps of $W^{\prime}$ consists of $p_{1}<p_{2}<\cdots<p_{m}$. Assume that $u$ is a admissible evolving crystal in a time interval $J$, i.e.,

(a) $u(t, \cdot)$ is piecewise linear whose slope consists of $p_{i}$ 's;

(b) for each $t \in J$ if $u(t, \cdot)$ has slope $p_{i}$ on an interval then the slope of $u(t, \cdot)$ in an adjacent neighborhood is either $p_{i+1}$ or $p_{i-1}$;

(c) $u$ is continuous in space-time and (the abscissa of a jump of $u_{\infty}(t, \cdot)$ moves smoothly in time $t \in J$; each jump does not collide each other.

We have abused the use of word 'crystal' to represent functions rather than curves. Using the similar argument in deriving (1.5) we have (1.5) on each space (maximal) interval $I_{j}(t)=\left(\alpha_{j}(t), \beta_{j}(t)\right)$, where $u_{x}(t, \cdot)$ is constant $p_{i}$ and

$$
\Delta=W^{\prime}\left(p_{i}+0\right)-W^{\prime}\left(p_{i}-0\right), L=\text { the length of } I_{j}(t) ;
$$

the transition number $\chi=1$ if both $u_{x}\left(\alpha_{j}-\delta\right)$ and $u_{\infty}\left(\beta_{j}+\delta\right)$ are greater than $p_{i}$ (for small $\delta$ ); $\chi=-1$ if both are smaller than $p_{i} ; \chi=0$ otherwise. This (1.5) is the evolution law (for admissible evolving crystal) corresponding to (1.1) we are seeking for. It turns out that this equation (1.5) on each $I_{j}(t)$ yields a system of ordinary differential equations (ODE) for the end points of $I_{j}(t)$ or the length of $I_{j}(t)$ (cf. [T1], [T3], [AG1], [GirK1], [GMHG2]). If the set $\left\{I_{j}\right\}$ is finite or $u(t, \cdot)$ is periodic in $z$, then the system of (ODE) has only finitely many unknowns and is solvable locally in time. So in particular if initial data satisfies (a) and (b) and it is periodic in $x$, then there is an admissible crystal satisfying (1.5) (locally in time) with this initial data. Some of $I_{j}$ 's may disappear at the maximal time $t_{0}$, where the ODE system is solvable on $\left(0, t_{0}\right)$. Fortunately if $a$ is positive, $u\left(t_{0}, \cdot\right)$ fulfills (a) and (b) (at $t_{0}$ ) so one can again solve the ODE system with initial data $u\left(t_{0}, \cdot\right)$; repeating this argument we extend the solution globally in time [T3], [GirK1]. In the terminology of [GMHG2] there is a global weakly admissible evolving crystal satisfying (1.5) with given periodic initial data satisfying (a) and (b) with $t=0$ provided that $a>0$. The same argument applies to (1.2) with a trivial modification [GMHG2].

This approach is good especially for computational purpose. However, there arise at least two fundamental questions.

(I)(Starting from nonadmissible data). If initial data does not satisfy (a)-(b) i.e., it is nonadmissible, what is a natural formulation of solutions to (1.1) or (1.2)?

(II) (General W not necessarily piecewise linear). Is it possible to solve the initial value problem for (1.1) or (1.2) when $W^{\prime}$ has jumps but $W$ is not necessarily piecewise linear? For (1.1) Fukui and the second author [FG] introduced a new notion of solutions for general $W$ and general Lipschitz initial data by adapting the theory of nonlinear semigroups initiated 
by Kōmura[Ko]. For a periodic initial data they constructed a unique globalin-time solution to (1.1). The problems (I), (II) are settled in this case. They rewrote (1.1) as a divergence form

$$
u_{t}-\tilde{W}\left(u_{x}\right)_{x}=0
$$

by setting

$$
\tilde{W}(p)=\int_{0}^{p} a(q) W^{\prime \prime}(q) d q
$$

and applied the theory of subdifferential equations in periodic $L^{2}$ spaces to get solutions. We should ask whether or not a solution of [T1] and [AG1] is also a solution in [FG] when $W$ is piecewise linear. Fortunately, both solutions are consistent. Indeed, in [FG] it is shown that the time derivative of their solutions actually agrees with the one given by (1.5) if $u$ is piecewise linear.

Recently, Elliot, Gardiner and Shätzle [EGS] proved that a weakly admissible evolving crystals satisfying (1.5) on each linear portion of the graph of $u(t, \cdot)$ (called facet) is a solution in the sense of [FG] when $W$ is piecewise linear. The solution in [FG] is given by the limit of solution of (1.1) with $W$ replaced by a regularized $W^{\epsilon}$ approximating $W$. Thus the evolution law (1.5) for admissible evolving crystals is justified. The behavior of solutions of [FG] is studied both analytically and numerically in [EGS]. Although the theory in [FG] works well for (1.1), it seems very difficult to apply the theory to (1.2) under the presence of $C$ even if $C$ is a (nonzero) constant.

There is another justification of the evolution law (1.5) (for closed curves) by Almgren and Taylor [AT] when $W$ is piecewise linear. They investigated a semi-discretized implicit scheme introduced by [ATW]. The time is discretized and at each time step the value of solution is given by solving a (non-discretized) variational problem. Their scheme does not require that the solution is piecewise linear at each time step. Their approximate solutions converge as the time grid tends to zero (by taking a subsequence) for general interfacial energy [ATW]. The limit weak solution is called a flat curvature flow. In [AT] it is shown that a weakly admissible evolving crystal (of closed curves) is the unique flat curvature flow with the same initial data provided that two adjacent facets do not vanish simultaneously. This justifies (1.5). For smooth $W$ see also [FK] and [LS]. In particular, it is shown in [FK] that the limit is contained in the level set flow of [CGG] and [ES].

In this paper we introduce a new notion of solutions for (1.2) or its general form (1.3) with (1.4). Since our theory is interpreted as an extension of the theory of viscosity solutions [CIL], we should define sub- and supersolution of (1.3) for nonsmooth functions. The issue is what kind of class of test functions we choose so that we define weighted curvatures. We shall always 
assume that the set of jump discontinuities of $W^{\prime}$ is a discrete set $P$. For technical reasons we also assume that $W \in C^{2}(\mathbf{R} \backslash P)$ has bounded second derivatives on each bounded set in $\mathbf{R} \backslash P$.

We introduce the notion of $(P-)$ faceted functions in an open interval. Roughly speaking, a piecewise $C^{1}$ function $f \in C(\Omega)$ is $P$-faceted if for each $p \in P$ the set $\left\{x ; f^{\prime}(x)=p\right\}$ consists of a union of closed (nontrivial) intervals (called faceted region) and the transition number $\chi$ is well-defined on each faceted region. We then introduce the class of $P$-faceted $C^{2}$ functions on an open interval $\Omega$ so that weighted curvature $\Lambda_{W}$ is defined everywhere ( $\left.\$ 2\right)$. Let us give a definition of subsolution at least for continuous functions. We say a continuous function $u:[0, T) \times \bar{\Omega} \rightarrow \mathbf{R}$ is a subsolution of (1.3) in $Q=(0, T) \times \Omega$ if

$$
g^{\prime}(\hat{t})+F\left(\hat{t}, f^{\prime}(\hat{x}), \Lambda_{W}(f)(\hat{x})\right) \leq 0
$$

whenever $g \in C^{1}(0, T), f \in C_{P}^{2}(\Omega)$ satisfies

$$
\max _{Q}(u-\psi)=(u-\psi)(\hat{t}, \hat{\boldsymbol{x}}), \quad(\hat{t}, \hat{\boldsymbol{x}}) \in Q
$$

with $\psi(t, x)=g(t)+f(x)$ (called a test function at $(\hat{t}, \hat{x})$ ), where $C_{P}^{2}(\Omega)$ denotes the set of all $P$-faceted $C^{2}$ functions on $\Omega$. If $f^{\prime}(\hat{x}) \in P$, as already explained we set

$$
\Lambda_{W}(f)(\hat{x})=\chi \Delta / L
$$

where $L$ is the length of faceted region containing $\hat{x}$. If $f^{\prime}(\hat{x}) \notin P$, then we set

$$
\Lambda_{W}(f)(\hat{x})=W^{\prime \prime}\left(f^{\prime}(\hat{x})\right) f^{\prime \prime}(\hat{x}) .
$$

It is standard to extend this definition to semicontinuous functions as in $\$ 2$. A supersolution is defined in the similar way. By a generalized solution we mean a sub- and supersolution. It turns out that a generalized solution is consistent with an admissible solution in [T1] and [AG1] when $W$ is piecewise linear. Indeed, it is shown in [GMHG2] that a weakly admissible evolving crystal satisfying (1.5) in each faceted region is a generalized solution in this sense. The argument can be extended to (1.2) with extra assumptions on $a$. When $C$ in (1.2) does depend on $x$, our present theory does not apply. Moreover, the assumption that $u_{t}$ is constant on $I(t)$ in deriving (1.5) seems to be not natural [R], [GMHG1]. We shall discuss this problem in a forthcoming paper.

1.2. Comparison results. It is always crucial to establish comparison principle in the theory of viscosity solutions. It is, modulo suitable assumptions, of the form: 
Comparison Principle. If $u$ and $v$ are sub-and supersolutions of (1.3) (with (1.4)) respectively in $Q=(0, T) \times \Omega$, then $u \leq v$ in $Q$ provided that $u \leq v$ on the parabolic boundary $\partial_{p} Q$ of $Q$.

We establish this comparison principle for a bounded open interval $\Omega$ under uniform continuity assumptions on $F$ in

$$
\left[0, T^{\prime}\right] \times[-K, K] \times \mathbf{R} \text { for each } T^{\prime}<T, K>0 .
$$

This result applies to (1.1) and (1.2) when $a \geq 0$ and $C$ are continuous in $\mathbf{R}$ and $[0, T)$, respectively.

The basic strategy of the proof is the same as that for the case when $W$ is smooth. However, several new ideas and extra work are necessary. This is why the proof is long ( $\$ 4-\$ 7)$. Since the standard maximum principle for semicontinuous functions [CIL] does not apply to handle our weighted curvature, we establish a maximum principle for faceted functions (\$4). To handle semicontinuous functions we need to regularize them by sup-convolutions [CIL]. Unfortunately, usual sup-convolutions are not good for our purpose. We introduce sup-convolution by faceted functions and study its properties in §5. A new aspect of our sup-convolution is that if $f$ takes a local maximum at $\hat{x}$ then its sup-convolution is faceted near $\hat{x}$ (Theorem 5.3). Usually, there is an equivalent definition of subsolutions using semijets. We have to introduce similar equivalent definitions of subsolutions. It is very sensitive how to define time semijets in a neighborhood of a faceted region so that both definitions of subsolutions are equivalent $(\$ 6)$. With these preparations we prove the comparison principle in $\$ 7$. Let us briefly explain the idea of our proof. We argue by contradiction. Using an extension of the method developed in [CGG] and [Go] we reduce our problem so that our sup-convolutions of both $u$ and $-v$ are faceted at a point we are interested in. We construct suitable test functions of both $u$ and $v$ from these sup-convolutions with help of equivalent definitions of solutions. Applying the maximum principle for faceted functions and (1.4), we get a contradiction. Note that our method does apply when $P$ is an infinite set. However, it seems difficult to extend our proof to unbounded $\Omega$ because so far the method in [CGG] and [Go] requires the boundedness of $\Omega$. If both functions $u$ and $v$ are periodic in space with the same period (independent of time) we have also comparison principle as shown in $\$ 7$.

Our comparison principle is totally new even if for (1.1) when $W^{\prime}$ has jumps. In [FG] a comparison principle is proved for their solutions for (1.1). It is obtained as a limit of approximate solutions satisfying comparison principle. Besides the difference of definitions of solutions, their results are weaker than ours since they assume both $u$ and $v$ are (periodic-in-space) solutions. In [GGu] maximum principle and comparison principle are proved for admissible evolving crystals. Although they handle curves of finitely many facets 
with no end points for comparison principle, their maximum principle applies to get comparison principle for weakly admissible evolving crystals satisfying

$$
u_{t}-a\left(u_{s}\right)\left(\Lambda_{W}(u)-C(t)\right)=0
$$

on each faceted region when $W$ is piecewise linear; this equation corresponds to (1.2), of course. (In [GGu] $C$ is assumed to be a constant but the method and result apply to nonconstant $C$ with trivial modifications). Our results are considered as a natural extension of this type of results since a weakly admissible evolving crystal satisfying (1.5) is a generalized solution [GMHG2].

If the singularities of $W \in C^{2}(\mathbf{R} \backslash P)$ are weak, for example, $W^{\prime}$ is Lipschitz, then the comparison principle for viscosity solutions has already been proved in [G]; see also [OhS] and [GSS] for evolutions of closed curves.

1.3. Existence results. In our formulation we shall prove the (unique) existence of a global-in-time, continuous, periodic-in-space, solution of (1.3) if initial data is continuous and periodic. This answers our questions (I) and (II) at least for periodic data. For the homogeneous Dirichlet problem on a bounded interval $\Omega$ of (1.3) we have some global existence result by reducing the problem to periodic situation. This result applies to (1.1). However, it does not apply to (1.2) even if $C$ is (nonzero) constant. This is not a technical restriction. In fact, even for smooth $W$ with $W^{\prime \prime}>0$, a local solution may break down at the boundary $\partial \Omega$ and boundary detachment phenomena may occur (cf. eg. $[K K]$ ). On the contrary our global solution for the Dirichlet problem actually attains zero at $\partial \Omega$ for all time. This is an intrinsic reason why our global result does not apply to (1.2). We do not pursue the boundary value problems in this paper except a few remarks.

To show existence results we establish the Perron type existence theorem to our equations. This is by now standard in the theory of viscosity solutions [CIL] for PDEs. However, in our setting we should prove it when the test function is faceted at a point of interest. Unfortunately, this is not a trivial modification of the standard results. We should modify the test function $\psi$ in a class of $C^{2} P$-faceted functions in space so that $\max _{Q}(u-\psi)$ is attained only at one time $\hat{t}$ and at one faceted region. We introduce canonical modification of $C^{2} P$-faceted functions for this purpose. Another property we use in the proof is

$$
W^{\prime \prime}\left(f^{\prime}\left(x_{n}\right)\right) f^{\prime \prime}\left(x_{n}\right) \rightarrow 0 \text { as } x_{n} \rightarrow x_{0}
$$

for $f \in C_{P}^{2}(\Omega)$, where $x_{0}$ is the boundary of a faceted region of $f$ and $f^{\prime}\left(x_{n}\right) \notin P$. This convergence follows from the fact that $W^{\prime \prime}$ is bounded in every bounded set in $\mathbf{R} \backslash P$ and that $f \in C_{P}^{2}(\Omega)$. We shall prove the Perron type existence results in $\$ 8$. In the last section we construct a sub- and supersolution for a given initial data. This together with the Perron type existence results and comparison results yield an existence result for periodic 
data. To construct sub- and supersolution we modify the method developed in [CGG] and improved by [IS].

Our existence result for general continuous (not necessarily Lipschitz) initial data (periodic in $x$ ) is new even for (1.1). The existence result in [FG] needs Lipschitz continuity of initial data. We do not know whether a solution in [FG] is continuous in space-time although it applies to all convex $W$. We believe our solution is consistent with a solution in [FG] although we do not prove in this paper.

When $W$ is piecewise linear a solution with nonadmissible data (question II) for (1.1) has been studied in [T3] and [EGS]. If initial data is piecewise linear nonadmissible, a 'solution' is constructed in [T3] by solving ODE. It turns out [EGS] that it is a solution in the sense of [FG] for (1.1). Even for some piecewise $C^{1}$ initial data, a solution given by ODE is proposed in [T3], [EGS]. However, initial data is very restrictive. Instead of proving that all proposed solution is a solution in our sense, we give an example of solutions with nonadmissible data in $\$ 2$.

1.4. Background of problems. Surface-energy-driven motion of interfaces attracts many mathematicians and physicists to study evolution of phase boundary $\Gamma_{t}$ such as a surface of crystal. Let $\mathbf{n}$ denote the unit normal vector field determining the orientation of $\Gamma_{t}$. We assume that $\Gamma_{t}$ is a curve in the plane. Let $V$ denote the normal velocity in the direction of $n$. If $V$ depends on local geometry, a typical evolution equation is of the form

$$
V=-\frac{1}{\beta(\mathbf{n})}\left(\sum_{i=1}^{2} \frac{\partial}{\partial x_{i}}\left(\partial_{i} \gamma(\mathbf{n})\right)+C(t)\right) \text { on } \Gamma_{t} .
$$

Here $\gamma: \mathbf{R}^{2} \rightarrow \mathbf{R}$ is of the form

$$
\gamma(q)=|q| \gamma_{0}(q /|q|), \quad q=\left(q_{1}, q_{2}\right) \in \mathbf{R}^{2}, q \neq 0
$$

and $\gamma_{0}, \beta$ are positive functions defined on the unit circle. In (1.6) $\partial_{i} \gamma$ denotes the partial derivative $\partial \gamma / \partial q_{i}$ as a function on $\mathbf{R}^{2}$. The quantity $\gamma_{0}$ is called the interfacial energy density, while $\beta$ is called the kinetic coefficient. The function $C(t)$ is given. Physically it describes the bulk free energy of crystal relative to that of the other phase. Typically, this is the difference of temperature between two phases on $\Gamma_{t}$ or the difference of pressure. The equation (1.6) was derived mathematically by Angenent and Gurtin [AG1] (see also [Gu]) from axioms of continuum thermomechanical theory. In physical literature (1.6) was first formulated by Müller-Krumbhaar et al [MBK] as a gradient flow of free energy in the spirit of the time-dependent GinzburgLandan theory. This model is good if the crystal surrounded by $\Gamma_{t}$ is small so that surface effects dominate bulk effects, since in this case we may assume 
that bulk energy $C(t)$ is given and independent of the space variable. If $\gamma_{0}$ is a positive constant, $\gamma_{0}$ is called isotropic. If both $\gamma_{0}$ and $\beta$ are isotropic, (1.6) with $C=0$ becomes the famous curve shortening equation. We focus the initial value problem for anisotropic curve shortening equation (1.6) when the interfacial energy may have singularities. To classify the problems it is convenient to recall the Frank diagram of $\gamma_{0}$ :

$$
\mathcal{F}=\left\{\left(q_{1}, q_{2}\right) \in \mathbf{R}^{2} ; \gamma(q)=1\right\}
$$

We always assume that $\mathcal{F}$ is convex, so that (1.6) is degenerate parabolic at least formally. There are three typical situations as explained in [GMHG1].

(1) $\mathcal{F}$ is smooth and has positive curvature; $\gamma_{0}$ is called a strict convex smooth energy.

(2) $\mathcal{F}$ is at most $C^{1,1}$ and $C^{2}$ except finitely many points. The curvature of $\mathcal{F}$ is bounded but may be zero somewhere; $\gamma_{0}$ is called a singular energy without corners.

(3) $\mathcal{F}$ is $C^{2}$ except finitely many points but is not $C^{1}$. The curvature of $\mathcal{F}$ is bounded but may be zero somewhere; $\gamma_{0}$ is called an energy with corners.

An isotropic energy is a typical example of a strictly convex smooth energy. In the case of (1) the equation (1.6) is quasilinear (nondegenerate) parabolic so the classical theory [LSU] applies to get local-in-time smooth solution to (1.6) provided that $\beta$ is smooth. Even if $C$ exists, it is possible to extend the solution globally in time in the level set sense ([CGG], [ES]). Note that the level set method applies to (1.6) if $\gamma_{0}$ is $C^{2}$ and convex; the strictly convexity is unnecessary [CGG], [GGo]. There are by now extensive literature to the case (1). We suggest the reader to consult a book [Gu], the review by Taylor, Cahn and Handwerker [TCH] and the recent review [GMHG1] and the references therein.

Even in the case of (2) the level set method does apply to get global generalized solutions ([OhS], [GSS]). A local existence of "strong" solutions is established by [AG2]. If $\Gamma_{t}$ is given as a graph of $y=u(t, x)$, then (1.6) becomes

$$
u_{t}-a\left(u_{\infty}\right)\left(\left(W^{\prime}\left(u_{\infty}\right)\right)_{\infty}-C(t)\right)=0
$$

with

$$
\begin{aligned}
& a(p)=\left(1+p^{2}\right)^{1 / 2} M(p) \\
& 1 / M(p)=\beta\left(-p /\left(1+p^{2}\right)^{1 / 2},\left(1+p^{2}\right)^{-1 / 2}\right) \\
& W(p)=\gamma(-p, 1)
\end{aligned}
$$


Here (2) is equivalent to say that $W$ is $C^{1,1}$ and $C^{2}$ except finitely many points. In [G] properties of global generalized solution are studied as well as comparison and existence results. See [GMHG1] and the references therein.

In the case of (3) the level set method does not apply so far. We discuss the case when $\Gamma_{t}$ is represented as the graph of functions. Our $W$ in (1.8) satisfies our assumption of regularity if (and only if) $\gamma_{0}$ is an energy with corners. This paper extends the theory of viscosity solutions to establish fundamental comparison and existence of solutions so that it applies to this setting.

A typical example of an energy with corners is a crystalline energy, where $\mathcal{F}$ is assumed to be a convex polygon. For (1.7) this is the case when $W$ is piecewise linear with finite jumps of $W^{\prime}$. As mentioned before its evolution law (1.6) is reduced to a system of ODEs for admissible evolving crystals. This is first observed by Taylor [T1] (for $\beta \cdot \gamma=$ const.) and independently by Angenent and Gurtin [AG1]. Their evolution is qualitatively similar as the case when $\gamma_{0}$ is smooth. For example we have comparison principle for admissible evolving crystals 'solving' (1.6) [GGu]. If $\beta \cdot \gamma=$ const. and $C \equiv 0$, a closed convex admissible evolving crystal shrinks to a point and the way of shrinking is asymptotically similar to the Wulff shape of $\gamma$ provided that initial polygon has more than five corners [St] as conjectured by Taylor [T1]. Although it is interesting to study the behavior of our solutions, we do not discuss them in this paper. Recently, surface evolutions by crystalline energy is analysed by [GGuM]. Among other results comparison principle for admissible evolution is established there. For the background of a crystalline energy see the review of Taylor [T2].

It is a geometric natural idea to approximate a strictly convex smooth energy by a crystalline one. In fact, this approximation has been used in calculation of curve evolutions. If $\Gamma_{t}$ is a graph, the convergence result of [FG] applies to (1.1). It says that approximate solution actually converges to the true solution with no convergence rate. At the same time Girão and Kohn [GirK1] studied this problem and obtained the convergence rate in the Sobolev space $H^{1}$ (for Dirichlet and Neumann problems). For convex closed curves Girão [Gir] obtained a convergence rate in the topology of Hausdorff distance. Recently this result is extended by Yazaki for the equation $V=-(\operatorname{div} \mathbf{n})^{\alpha}$ on $\Gamma_{t}$ with $\alpha>0$. See also the review paper [GirK2]. Unfortunately, these works do not apply if $C$ exists in (1.2). We shall discuss convergence result even for general equations (1.3) in the realm of viscosity solutions in a forthcoming paper.

In the crystal growth problem there often arises an energy $\gamma_{0}$ with corners so that its Wulff shape has a flat portion called a facet. It is natural to consider such a $\gamma_{0}$ if the temperature is lower than roughening temperature. Although this phenomena is common in all crystal growth [Ch], a typical 
example is the growth of crystals of Helium. If facets exists in evolving crystals, it is explained in physics [Ch] that the velocity on facets is not proportional to the chemical potential difference $\Delta \mu$ which is the weighted curvature plus the pressure difference, although the velocity is nondecreasing in $\Delta \mu$ and it is zero for $\Delta \mu=0$. If $\Delta \mu$ is small, the velocity may be zero in some situation. This is a reason why we consider

$$
u_{t}+F\left(u_{z}, \Lambda_{W}(u)+C\right)=0
$$

or its more general from (1.3) instead of (1.2).

\section{§2. Definition of generalized solutions.}

This section establishes conventions of notation and introduces several notions of functions and weighted curvature. The goal of this section is to define generalized solutions for evolution equations with singular interfacial energy.

2.1. Assumption (Set $P$ and function $W$ ). Let $P$ be a closed discrete set in $\mathbf{R}$. In other words $P$ is either a finite set or a countable set having no accumulation points in $\mathbf{R}$. If $P$ is nonempty, $P$ is of form $\left\{p_{j}\right\}_{j=1}^{m},\left\{p_{j}\right\}_{j=-\infty}^{\infty}$, $\left\{p_{j}\right\}_{j=-\infty}^{-1}$, or $\left\{p_{j}\right\}_{j=1}^{\infty}$ with $\lim _{j \rightarrow \infty} p_{j}=\infty, \lim _{j \rightarrow-\infty} p_{j}=-\infty$, where $p_{j}$ 's are indexed in increasing order $p_{j}<p_{j+1}$ and $m$ is a positive integer.

Let $W$ be a convex function on $\mathbf{R}$ with values in $\mathbf{R}$. We assume that $W$ is $C^{2}$ outside $P$. Moreover, we assume that $W^{\prime \prime}$ is bounded in any compact set except all points in $P$.

Throughout this paper we always assume these assumptions concerning $P$ and $W$.

2.2. Definition ( $P$-faceted). Let $\Omega$ be an open interval. A function $f$ in $C(\Omega)$ is called faceted at $x_{0}$ with slope $p$ in $\Omega$ if $f$ fulfills the following conditions.

There are a closed nontrivial finite interval $I(\subset \Omega)$ containing $x_{0}$ such that $f$ agrees with an affine function

$$
\ell_{p}(x)=p\left(x-x_{0}\right)+f\left(x_{0}\right)
$$

in $I$ and $f(x) \neq \ell_{p}(x)$ for all $x \in J \backslash I$ with some neighborhood $J(\subset \Omega)$ of $I$. The interval $I$ is called a faceted region of $f$ containing $x_{0}$ and is denoted by $R\left(f, x_{0}\right)$.

A function $f$ is called $P$-faceted at $x_{0}$ in $\Omega$ if $f$ is faceted at $x_{0}$ in $\Omega$ with some slope $p$ belonging to $P$.

2.3. Definition (Weighted curvature). Let $x_{0}$ be a point in $\Omega$. For $f \in C(\Omega)$ we set the value

$$
\Lambda_{W}\left(f, x_{0}\right)=W^{\prime \prime}\left(f^{\prime}\left(x_{0}\right)\right) f^{\prime \prime}\left(x_{0}\right)
$$


if $f$ is second differentiable at $x_{0}$ and $f^{\prime}\left(x_{0}\right) \notin P$ and

$$
\Lambda_{W}\left(f, x_{0}\right)=\frac{\chi}{I} \Delta_{i}
$$

if $f$ is $P$-faceted at $x_{0}$ in $\Omega$ with slope $p_{i}$, where $\Delta_{i}=W^{\prime}\left(p_{i}+0\right)-W^{\prime}\left(p_{i}-0\right)$. We call the value $\Lambda_{W}\left(f, x_{0} ; c\right)$ weighted curvature of $f$ at $x_{0}$. This value is invariant under the addition of affine functions to $W$. Here $L=L\left(f, x_{0}\right)$ is the length of the faceted region $I$ containing $x_{0}$ and $\chi=\chi\left(f, x_{0}\right)$ is the transition number defined by

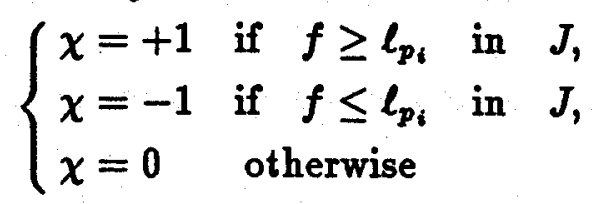

for some neighborhood $J$ of the facet region $I$. We often write $\Lambda_{W}\left(f, x_{0}\right)$ by $\Lambda_{W}(f)\left(x_{0}\right)$ if we emphasize that this quantity is a function of $x_{0}$.

For later convenience, here we introduce the left transition number $\chi_{-}=$ $\chi_{-}\left(f, x_{0}\right)$, and the right transition number $\chi_{+}=\chi_{+}\left(f, x_{0}\right)$ by

$$
\left\{\begin{array}{l}
x-=+1 \text { if } f \geq \ell_{p_{i}} \text { in }\left\{x \in J ; x \leq x_{0}\right\} \\
\chi_{-}=-1 \text { if } f \geq \ell_{p_{i}} \text { in }\left\{x \in J ; x \leq x_{0}\right\}
\end{array}\right.
$$

and

$$
\left\{\begin{array}{l}
\chi_{+}=+1 \text { if } f \geq \ell_{p_{i}} \text { in }\left\{x \in J ; x \geq x_{0}\right\} \\
\chi_{+}=-1 \text { if } f \geq \ell_{p_{i}} \text { in }\left\{x \in J ; x \geq x_{0}\right\}
\end{array}\right.
$$

respectively. By definition we see $\chi=\left(\chi_{+}+\chi_{-}\right) / 2$.

2.4. Definition (Space of admissible functions). A function $f \in C^{2}(\Omega)$ belongs to a class $C_{P}^{2}(\Omega)$ if $f$ is $P$-faceted at $x_{0}$ in $\Omega$ whenever $f^{\prime}\left(x_{0}\right)$ belongs to $P$.

Let $T$ be a positive number. For $Q=(0, T) \times \Omega$ with $T>0$ let $A_{P}(Q)$ be the set of functions on $Q$ of the form

$$
f(x)+g(t), \quad f \in C_{P}^{2}(\Omega), \quad g \in C^{1}(0, T) .
$$

An element of $A_{P}(Q)$ is called an admissible function.

2.5. Assumptions (Function $F$ ). Let $F$ be a function from $[0, T) \times \mathbf{R} \times \mathbf{R}$ to $\mathbf{R}$. We often assume

$F$ is continuous in $[0, T) \times \mathbf{R} \times \mathbf{R}$ with values in $\mathbf{R}$,

$$
F(t, p, X) \leq F(t, p, Y)
$$

$$
\text { for } X \geq Y, t \in[0, T), p \in \mathbf{R} \text { (degenerate ellipticity), }
$$

For each $K>0$ and $T^{\prime}<T$,

$F$ is uniformly continuous in $\left[0, T^{\prime}\right] \times[-K, K] \times \mathbf{R}$. 
We explicitly state these assumptions in our statements if we need to assume them.

We are now in position to define our generalized solution in the viscosity sense.

2.6. Definition (Generalized solution). A real valued function $u$ on $Q$ is a (viscosity) subsolution of

$$
u_{t}+F\left(t, u_{\infty}, \Lambda_{W}(u)\right)=0 \quad \text { in } Q
$$

if the uppersemicontinuous envelope $u^{*}<\infty$ in $[0, T) \times \bar{\Omega}$ and

$$
\psi_{t}(\hat{t}, \hat{x})+F\left(t, \psi_{\infty}(\hat{t}, \hat{x}), \Lambda_{W}(\psi(\hat{t}, \cdot), \hat{x})\right) \leq 0
$$

whenever $(\psi,(\hat{t}, \hat{z})) \in A_{P}(Q) \times Q$ fulfills

$$
\max _{\boldsymbol{Q}}\left(u^{*}-\psi\right)=\left(u^{*}-\psi\right)(\hat{t}, \hat{x})
$$

Here

$$
u^{*}(t, x)=\lim _{c \downarrow 0} \sup \{u(s, y) ;|s-t|<\varepsilon,|x-y|<\varepsilon,(s, y) \in Q\}
$$

for $(t, x) \in \bar{Q}$ and $u_{*}=-(-u)^{*}$. A (viscosity) supersolution is defined by replacing $u^{*}(<\infty)$ by the lowersemicontinuous envelope $u_{*}(>-\infty)$, max by $\mathrm{min}$ and the inequality in (2.1) by the opposite one. If $u$ is sub- and supersolution, $u$ is called a viscosity solution or a generalized solution. Hereafter we avoid to use the word viscosity. Function $\psi$ satisfying (2.2) is called a test function of $u$ at $(\hat{t}, \hat{x})$.

The following propositions are easily derived.

2.7. Proposition (Addition by affine functions). Let $\Omega$ be an open interval. Let $u$ be a sub- (resp. super-) solution of $(E)$. Then $v(t, x)=$ $u(t, x)-\ell(x)$ is a sub- (resp. super-) solution of

$$
v_{t}+F\left(t, v_{x}+A, \Lambda_{W_{A}}(v)\right)=0
$$

where

$$
\begin{aligned}
& \ell(x)=A x+B \text { for some real number } A \text { and } B \\
& W_{A}(q)=W(q+A) \text { for all } q \in \mathbf{R} \\
& P_{A}=\{p-A ; p \in P\}
\end{aligned}
$$


2.8. Proposition. If $\psi \in A_{P}(Q)$ satisfies (2.1) at each point $(\hat{t}, \hat{x}) \in Q$, then $\psi$ is a subsolution of (E) in $Q$ provided that (F2) holds.

2.9. Example of equations. Let a function $F$ be of form

$$
F(t, p, X)=-a(p)(X+C(t, p))
$$

with a continuous nonnegative function $a$ and a continuous function $C$ in $[0, T) \times \mathbf{R}$. Then conditions (F1), (F2) and (F3) are fulfilled. The equation (E) becomes

$$
u_{t}-a\left(u_{\infty}\right)\left(\Lambda_{W}(u)+C\left(t, u_{\infty}\right)\right)=0 .
$$

The term $\Lambda_{W}(u)+C$ is called the weighted curvature with driving force and is denoted by $\Lambda_{W}(u ; C)$ as in [GMHG1]. A more general form of $F$ is

$$
F(t, p, X)=G(t, p, X+C(t, p)),
$$

where $G:[0, T) \times \mathbf{R} \times \mathbf{R} \rightarrow \mathbf{R}$. It is easy to see that $F$ fulfills (F1) (resp. (F3)) provided that $G$ satisfies (F1) (resp. (F3)) where $C$ is continuous in $[0, T) \times \mathbf{R}$. Clearly if $G$ satisfies (F2), so does $F$. The equation (E) now becomes

$$
u_{t}+G\left(t, u_{x}, \Lambda_{W}(u)+C\left(t, u_{x}\right)\right)=0 .
$$

Such a type of equation is important to describe evolutions of crystals of Helium below the roughening temperature (where $C$ is independent of $p$ ). As pointed out in [R] when $C$ depends on spatial variable $x$, it is not natural to define $\Lambda_{W}(u, C)$ just as a sum $\Lambda_{W}(u)+C$.

2.10. Remark. The equation (2.3) has many representation by use of different W. For example, if

$$
a(p) W^{\prime \prime}(p)=a_{0}(p) W_{0}^{\prime \prime}(p)
$$

in the distribution sense with some nonnegative continuous function $a_{0}$ and convex function $W_{0}$, then (2.3) can be formally written as

$$
u_{t}-a_{0}\left(u_{\infty}\right) \Lambda_{W_{0}}(u)-a\left(u_{\infty}\right) C\left(t, u_{\infty}\right)=0,
$$

which is also an example of (E). Fortunately, it is not difficult to see that $u$ is a subsolution of (2.3) if and only if $u$ is a subsolution of (2.5). So our definition of solutions is independent of representation of the equation. By the way there are many choices of $a_{0}$ and $W_{0}$ satisfying (2.4). Indeed, for a given $a_{0}(p)$, we have $W_{0}$ satisfying (2.4) by defining $W_{0}$ by

$$
W_{0}(p)=\int^{p} \int^{q} a(z) W^{\prime \prime}(z) / a_{0}(z) d z d q .
$$


2.11. Examples of solutions when $W$ is piecewise linear. We next consider several special solutions of (2.3) when $a>0$ and $W$ is piecewise linear. We say a function $v$ on $\mathbf{R}$ is an admissible crystal if $v$ is $P$-faceted at any point of $\mathbf{R}$ and the slopes of adjacent faceted region should be adjacent in $P$. This definition is the same as in [GMHG2]. Let $u$ be an admissible evolving crystal on a time interval $I^{\prime}$, i.e. $u(t, \cdot)$ is an admissible crystal with $u \in C\left(I^{\prime} \times \mathbf{R}\right)$ and jumps of $u_{\infty}$ moves smoothly in time for $t \in I^{\prime}$ and does not collide each other. For an admissible evolving crystal $u$ the equation (2.3) has a meaning on each faceted region of $u(t, \cdot)$. This equation agrees with those derived by [T1] and [AG1] in a little bit different setting and by [GirK1] in this setting (actually with $C=0$ and finite $P$ but these restrictions are inessential). In [GMHG2] we prove that an admissible evolving crystal $u$ in $(0, T) \times \mathbf{R}$ solving (2.3) is a generalized solution of (2.3) with $Q=$ $(0, T) \times \mathbf{R}$ if $C=0$. The same assertion is still valid under some reasonable assumption on $a$ even if $C$ does not vanish identically. In [GMHG2] $P$ was assumed to be finite but the proof works for general $P$. Even if $u$ is a weakly admissible evolving crystal, i.e. $u$ is an admissible evolving crystal on $\left[t_{h}, t_{h+1}\right), k=-1,0, . ., h$ for some $0=t_{-1}<t_{0}<. .<t_{h+1}=T$ and $u$ is continuous across $t=t_{k}, k=0, . ., h$, then $u$ is a generalized solution of (2.3) with $\Omega=\mathbf{R}$ provided that $u$ solves (2.3) on $\left(t_{h}, t_{k+1}\right), k=-1,0, . ., h$ (under some assumptions on $a$ unless $C=0$ ). This is also proved in [GMHG2]. In the definition of weakly admissible evolving crystals of [GMHG2], the open interval $\left(t_{\ell}, t_{\ell+1}\right)$ should be replaced by $\left[t_{\ell}, t_{\ell+1}\right)$.

In many cases for a given initial data $u_{0}$ which is an admissible crystal, we see there is a unique global-in-time weakly admissible evolving crystal $u$ satisfying (2.3) on $\left(t_{k}, t_{h+1}\right), k=-1,0, . ., h$ by solving a system of ordinary differential equations. A typical situation is that $u_{0}$ is periodic in $x$. Since a weak admissible evolving crystal solving (2.3) is our generalized solution of (2.3), there are many nontrivial examples of solutions.

2.12 Examples of solutions with nonadmissible initial data. When $W$ is piecewise linear, a solution with nonadmissible data for (1.1) has been studied in [T3] and [EGS]. For piecewise linear but nonadmissible initial data a solution is constructed in [T3] by solving ODE's. However, if initial data is not piecewise linear, creation of new facets also may occur but such an example is not well examined although there is a heuristice explanation in [T3].

We here give a simple examle of solution with such type of initial data for (1.1) with $W(p)=c|p|$ with $c>0$ :

$$
u_{t}-c a\left(u_{x}\right)\left(\left|u_{x}\right|\right)_{s}=0
$$

where $a \geq 0$ is continuous; we assume $a(0)>0$ since otherwise (2.6) becomes 
$u_{t}=0$ everywhere. We consider initial data $u_{0} \in C(\mathbf{R})$ of the form

$$
u_{0}(x)= \begin{cases}A(x), & c \leq \alpha_{0} \\ h_{0}, & \alpha_{0} \leq x \leq \beta_{0} \\ B(x), & \beta_{0} \leq x\end{cases}
$$

with

$$
\begin{aligned}
& \alpha_{0} \leq \beta_{0}, \\
& A \in C^{1}\left(-\infty, \alpha_{0}\right) \cap C\left(-\infty, \alpha_{0}\right], \quad A^{\prime}<0, \\
& B \in C^{1}\left(\beta_{0}, \infty\right) \cap C\left[\beta_{0}, \infty\right), \quad B^{\prime}>0, \\
& A\left(\alpha_{0}\right)=B\left(\beta_{0}\right)=h_{0} \quad \text { so that } u_{0} \text { is continuous. }
\end{aligned}
$$

We set

$$
D(k)=\int_{h_{0}}^{k}\left\{B^{-1}(\eta)-A^{-1}(\eta)\right\} d \eta,
$$

where $A^{-1}, B^{-1} \in C^{1}\left(h_{0}, \infty\right) \cap C\left[h_{0}, \infty\right)$ denote the inverse functions of $A$ and $B$, respectively. Note that

$$
B^{-1}(\eta)>\beta_{0} \geq \alpha_{0}>A^{-1}(\eta), \quad \eta>h_{0}
$$

so that $D^{\prime}(k)>0$ for $k>h_{0}$. The value $D(k)$ is the area enclosed by $y=k$ and $y=u_{0}(x)$. The inverse function $D^{-1}$ is well defined and $D^{-1} \in$ $C^{1}\left(h_{0}, \infty\right) \cap C\left[h_{0}, \infty\right)$. We then set $u \in C(\bar{Q})(Q=(0, \infty) \times \mathbf{R})$ of form

$$
u(t, x)= \begin{cases}A(x), & x \leq \alpha(t) \\ h(t), & \alpha(t) \leq x \leq \beta(t) \\ B(x), & \beta(t) \leq x\end{cases}
$$

with

$$
\begin{aligned}
& h(t)=D^{-1}(2 a(0) c t) \\
& \begin{cases}\alpha(t)=A^{-1}(h(t)), & \alpha(0)=\alpha_{0}, \\
\beta(t)=B^{-1}(h(t)), & \beta(0)=\beta_{0} \quad \text { with } \alpha_{0} \leq \beta_{0} .\end{cases}
\end{aligned}
$$

Since $h(t)>h_{0}$ for $t>0$ and

$$
B^{-1}(\eta)>\beta_{0} \geq \alpha_{0}>A^{-1}(\eta), \quad \eta>h_{0},
$$

we see $\alpha(t)<\beta(t)$ for $t>0$. Thus the function $u$ is well defined and $u \in C(\bar{Q})$ since $h \in C[0, \infty)$ so that $\alpha, \beta \in C[0, \infty)$. The interval $[\alpha(t), \beta(t)]$ is a faceted 
region of $u(t, \cdot), t>0$ and $\alpha^{\prime}(t)<0<\beta^{\prime}(t)$ for $t>0$. To convince the reader that (2.8) solves (2.6) we calculate $u_{t}$ on $(\alpha(t), \beta(t))$. By (2.9), (2.10) and definition of $D$ we see

$$
\beta(t)-\alpha(t)=A^{-1}(h(t))-B^{-1}(h(t))=D^{\prime}(h(t)), \quad t>0 .
$$

Since

$$
u_{t}(t, x)=h^{\prime}(t) \quad \text { for } x \in(\alpha(t), \beta(t))
$$

this yields

$$
\begin{aligned}
u_{t}(t, x) & =h^{\prime}(t)=\left(D^{-1}\right)^{\prime}(2 a(0) c t) \cdot 2 a(0) c \\
& =\frac{2 a(0) c}{D^{\prime}(h(t))}=\frac{2 a(0) c}{\beta(t)-\alpha(t)}=a(0) \Lambda_{W}(u(t, \cdot), x)
\end{aligned}
$$

where $W(p)=c|p|$.

Lemma. The function $u \in C(\bar{Q})$ in (2.8) is a generalized solution of (2.6) in $Q$. Its initial data is $u_{0}$ in (2.7). Moreover $-u$ is a generalized solution of (2.6) in $Q$ with initial data $-u_{0}$.

This is true even if $\alpha_{0}=\beta_{0}$. In this case a new faceted region $[\alpha(t), \beta(t)]$ is created instentaneously. The proof of Lemma is easy but we give it for completeness.

Proof. Step 1. We shall show that: the function $u$ is a subsolution of (2.6) in $Q$.

Let $\psi(t, x)$ be of form

$$
\psi(t, x)=f(x)+g(t) \quad f \in C_{P}^{2}(\mathbf{R}), \quad g \in C^{1}(0, \infty)
$$

and suppose that

$$
\max _{Q}(u-\psi)=(u-\psi)(\hat{t}, \hat{x}), \hat{t}>0
$$

we do not take $u^{*}$ since $u$ is continuous. If $f^{\prime}(\hat{x})=0$, then $f$ is faceted at $\hat{x}$ and by geometry we see

$$
\begin{aligned}
& \Lambda_{W}(u(\hat{t}, \cdot), \hat{x}) \leq \Lambda_{W}(f, \hat{x}) \\
& g^{\prime}(\hat{t})=h^{\prime}(\hat{t})
\end{aligned}
$$

By (2.11) we have

$$
g^{\prime}(\hat{t})-a(0) \Lambda_{W}(f, \hat{x}) \leq h^{\prime}(\hat{t})-a(0) \Lambda_{W}(u(\hat{t}, \cdot), \hat{x})=0
$$


If $f^{\prime}(\hat{x}) \neq 0$ so that $\hat{x}<\alpha(\hat{t})$ or $\hat{z}>\beta(\hat{t})$, then

$$
\Lambda_{W}(f, \hat{x})=0
$$

since $W^{\prime \prime}(p)=0$ for $p \neq 0$. Clearly $g^{\prime}(\hat{t})=u_{t}(\hat{t}, \hat{x})=0$ so we have

$$
g^{\prime}(\hat{t})-a(0) \Lambda_{W}(f, \hat{x})=0
$$

in this case. Since $u \in C(\bar{Q}), u$ is now a subsolution of (2.6) in $Q$.

Step 2. We shall show that: the function $u$ is a supersolution of (2.6) in $Q$.

Let $\psi(t, x)$ be as in (2.12) and suppose that

$$
\min _{\boldsymbol{Q}}(u-\psi)=(u-\psi)(\hat{t}, \hat{z}), \quad \hat{t}>0
$$

(1) If $f^{\prime}(\hat{x})=0$ and $\hat{x} \neq \alpha(t), \hat{x} \neq \beta(\hat{t})$, then

$$
\begin{aligned}
& \Lambda_{W}(u(\hat{t}, \cdot), \hat{x}) \geq \Lambda_{W}(f, \hat{x}), \\
& g^{\prime}(\hat{t})=h^{\prime}(\hat{t}),
\end{aligned}
$$

so that by (2.11) we have

$$
g^{\prime}(\hat{t})-a(0) \Lambda_{W}(t, \hat{x}) \geq h^{\prime}(\hat{t})-a(0) \Lambda_{W}(u(\hat{t}, \cdot), \hat{x})=0 .
$$

(2) If $f^{\prime}(\hat{x}) \neq 0$ and $\hat{x} \neq \alpha(\hat{t}), \hat{x} \neq \beta(\hat{t})$, then

$$
\Lambda_{W}(f, \hat{x})=0 \text { and } g^{\prime}(\hat{t})=0
$$

as in Step 1. Thus

$$
g^{\prime}(\hat{t})-a(0) \Lambda_{W}(f, \hat{x})=0-0=0 .
$$

(3) It remains to study the case when either $\hat{x}=\alpha(\hat{t})$ or $\hat{z}=\beta(\hat{t})$. If $f^{\prime}(\hat{x})=0$ and $\operatorname{int} R(f, \hat{x})$ intersects $(\alpha(\hat{t}), \beta(\hat{t}))$, then the situation is reduced to (1) so we may always assume

$$
\Lambda_{W}(f, \hat{x}) \leq 0
$$

including the case that $f^{\prime}(\hat{x}) \neq 0$ (which implies $\Lambda_{W}(f, \hat{x})=0$ ). We may assume $\hat{\boldsymbol{x}}=\alpha(\hat{t})$ since the case $\hat{\boldsymbol{\beta}}(\hat{t})$ can be treated in the same way. Since $u(\alpha(\hat{t}), t)=A(\alpha(\hat{t}))$ for $t<\hat{t},(2.13)$ implies $g^{\prime}(\hat{t}) \geq 0$. This now yields

$$
g^{\prime}(\hat{t})-a(0) \Lambda_{W}(f, \hat{x}) \geq 0
$$


Note that $g^{\prime}(\hat{t})$ may not equal $h^{\prime}(\hat{t})$ when $f^{\prime}(\hat{x}) \neq 0$. Since $(2.6)$ is invariant if we replace $u$ by $-u,-u$ also solves (2.6).

Clearly $u(0, x)=u_{0}(x)$ so the proof is now complete.

Remark (i) In [EGS] a piecewise $C^{1}$ initial data $u_{0}$ with finitely many $P$ -faceted regions was considered. Outside faceted regions $u_{0}$ was essential to be $C^{1}$ and $u_{0}^{\prime} \notin P$. They gave a local-in-time solution by solving ODE and proved that their solution is a solution in the sense of [FG]. we remark that our Lemma essentially implies that their solution is our generalized solution. Note that the situation is localized near each facet so the proof of our Lemma applies. Our Lemma also allows the situation that new facets are created instantaneously.

(ii) Our soltuion (2.8) is not $C^{1}$ in space for all $t>0$ even if initial data $u_{0}$ is smooth. This shows that the solution $u(t, \cdot)$ may lose smoothness instanteneously. When $\alpha_{0}=\beta_{0}(=0)$ the growth of the facet depends on the behavior of $A$ ande $B$ near $\alpha_{0}=0$. To see this we for simplicity assume that $B(x)=m x^{r}, A(x)=B(-x) \quad(m, r>0)$ and $h_{0}=0$. A direct computation shows that

$$
D(k)=\frac{2}{m} \int_{0}^{h} \eta^{1 / r} d \eta=c^{-1} d_{0} k^{1+1 / r}, \quad d_{0}=\frac{2 r}{1+r}
$$

which yields

$$
\begin{aligned}
& h(t)=\frac{m}{d_{0}}\{2 a(0) c t\}^{r /(1+r)} \\
& \beta(t)=m^{-1+1 / r} d_{0}^{-1 / r}\{2 a(0) c t\}^{1 /(1+r)}(=-\alpha(t)) .
\end{aligned}
$$

We conclude this section by giving another example of a solution of the equation

$$
u_{t}-a\left(u_{x}\right)\left(c\left|u_{x}-p_{0}\right|\right)_{x}=0
$$

for a given number $p_{0} \in \mathbf{R}$. We assume $a>0$ everywhere in this example. We consider (2.14) with initial data

$$
u_{0}(x)=\mu \sin (\nu x), \quad \mu>0, \quad \nu>0 .
$$

Assume that $0 \leq p_{0}<\mu \nu$. Similarly to the previous example one obtain an explicit form of the solution with initial data. Let $\alpha_{0}$ be the unique number that satisfies

$$
v_{0}^{\prime}\left(\alpha_{0}\right)=p_{0} \quad \text { with }-\pi / \nu<\alpha_{0}<0
$$

where

$$
v_{0}(x)=u_{0}(x)-p_{0} x
$$


Let $\alpha_{1}$ be the unique number that satisfies

$$
v_{0}\left(\alpha_{1}\right)=p_{0} \quad \text { with }-\pi / \nu \leq \alpha_{1}<0,
$$

so that $\alpha_{0} \leq \alpha_{1}$. We set

$$
\begin{aligned}
D(k) & =\int_{v_{0}\left(\alpha_{0}\right)}^{k}\left\{B^{-1}(\eta)-A^{-1}(\eta)\right\} d \eta \\
& =\int_{A^{-1}(k)}^{B^{-1}(k)}\left\{k-v_{0}(x)\right\} d x, \quad k \geq v_{0}\left(\alpha_{0}\right),
\end{aligned}
$$

where $A(x)=v_{0}(x)$ for $x, \alpha_{1} \leq x \leq \alpha_{0}$ and $B(x)=v_{0}(x)$ for $x, \alpha_{0} \leq x \leq 0$. The value $D(k)$ is the area enclosed by $y=k$ and $y=v_{0}(x)$ with $\alpha_{1}<x<0$. Let $T>0$ be the number defined by

$$
T=\frac{D(0)}{2 a\left(p_{0}\right) c}=\frac{1}{2 a\left(p_{0}\right) c}\left\{\frac{\mu}{\nu}\left(1-\cos \left(\mu \alpha_{1}\right)\right)+\frac{p_{0} \alpha_{1}^{2}}{2}\right\}
$$

We set

$$
h(t)=D^{-1}\left(2 a\left(p_{0}\right) c t\right) \quad \text { for } 0 \leq t \leq T,
$$

so that $h(0)=v_{0}\left(\alpha_{0}\right), h(T)=0, h^{\prime}(t)>0$ for $0<t<T$. We then set

$$
\begin{array}{ll}
\alpha(t)=A^{-1}(h(t)) & 0 \leq t \leq T \\
\beta(t)=B^{-1}(h(t)) & 0 \leq t \leq T
\end{array}
$$

so that

$$
\alpha(0)=\alpha_{0}, \quad \alpha(T)=\alpha_{1}, \quad \beta(0)=\beta_{0}, \quad \beta(T)=0 .
$$

As in the proof of Lemma one can prove that $2 \pi / \nu$-periodic (in space) function $u$ defined by

$$
\begin{aligned}
& u(t, x)= \begin{cases}u_{0}(x) & -\pi / \nu<x<\alpha(t), \\
u_{0}(\alpha(t))+p_{0}(x-\alpha(t)) & \alpha(t) \leq x \leq \beta(t), \\
u_{0}(x) & \beta(t)<x<-\beta(t), \\
u_{0}(-\alpha(t))+p_{0}(x+\alpha(t)) & -\beta(t) \leq x \leq-\alpha(t), \\
u_{0}(x) \quad \text { for } 0 \leq t \leq T, & -\alpha(t)<x \leq \pi / \nu\end{cases} \\
& u(t, x)=u(T, x) \text { for } t>T
\end{aligned}
$$

is the generalized solution of (2.14) in $Q=(0, \infty) \times \mathbf{R}$ with initial data $u_{0}(x)=\mu \sin (\nu x)$. Note that $u(t, x)$ becomes a stationary solution in a finite 
time. If $\left|p_{0}\right| \geq \mu \nu$, initial data $u_{0}(x)$ itself is the stationary solutions (If $-\mu \nu \leq p_{0} \leq 0$, then the solution is given by $u(t,-x+\pi / \nu)$, where $u$ is the solution of (2.14) with $p_{0}$ replaced by $\left.-p_{0}\right)$. If $p_{0}=0$, many quantities in the definition of $u$ are explicitly computable. For example $\alpha_{0}=-\pi /(2 \nu), \alpha_{1}=$ $-\pi / \nu$ so that

$$
\begin{aligned}
& A^{-1}(\eta)=-\{\arcsin (\eta / \mu)+\pi\} / \nu \\
& B^{-1}(\eta)=\arcsin (\eta / \mu) / \nu \\
& v_{0}\left(\alpha_{0}\right)=-\mu
\end{aligned}
$$

where arcsin is the principal value of the inverse of sin. We then calculate

$$
\begin{aligned}
D(k) & =\int_{-\mu}^{h}\left\{B^{-1}(\eta)-A^{-1}(\eta)\right\} d \eta \\
& =\frac{1}{\nu} \int_{-\mu}^{h}\left\{2 \arcsin \left(\frac{\eta}{\mu}\right)+\pi\right\} d \eta \\
& =\frac{1}{\nu}\left\{2\left[k \arcsin \left(\frac{k}{\mu}\right)+\sqrt{\mu^{2}-k^{2}}\right]+\pi k\right\}
\end{aligned}
$$

In particular $D(0)=2 \mu / \nu$, so that

$$
T=\frac{\mu}{\nu} \frac{1}{a(0) c} .
$$

In this case $u(T, x) \equiv 0$ so $u(t, x) \equiv 0$ for $t \geq T$.

§3. Main Theorems.

We state our comparison and existence results for the equation (E).

3.1. Comparison Theorem. Assume conditions (F1) and (F2). Assume (F3) if $F$ depends on the time variable $t$. Let $u$ and $v$ be a sub-, and supersolution of $(E)$, respectively, where $\Omega$ is a bounded open interval. If $u^{*} \leq v_{*}$ on the parabolic boundary $\partial_{p} Q(=[0, T) \times \partial \Omega \cup\{0\} \times \bar{\Omega})$ of $Q$, then $u^{*} \leq v_{*}$ in $Q$.

3.2. Comparison Theorem for periodic functions. Assume conditions (F1) and (F2). Assume (F3) if $F$ depends on $t$. Let $u$ and $v$ be a sub-, and supersolution of $(E)$ in $(0, T) \times \mathbf{R}$, respectively. Suppose that $u$ and $v$ are periodic in the spatial variable $x$ with period $\varpi$. If $u^{*} \leq v_{*}$ on $\{0\} \times \mathbf{R}$, then $u^{*} \leq v_{*}$ in $(0, T) \times \mathbf{R}$. 
3.3 Perron type Existence Theorem. Assume conditions (F1) and (F2). Let $u^{-}$and $u^{+}$be a sub-, and supersolntion of $(E)$ in $Q=(0, T) \times \Omega$, where $\Omega$ is an open interval, respectively. Suppose that $u^{-} \leq u^{+}$in $Q$ and $\left(u^{+}\right)^{*}<+\infty$ and $\left(u^{-}\right),>-\infty$ in $[0, T) \times \Omega$. Then there exists a generalized solution $u$ of (E) satisfying $u^{-} \leq u \leq u^{+}$in $Q$.

3.4. Perron type Existence Theorem for periodic functions. Assume conditions (F1) and (F2). Let $u^{-}$and $u^{+}$be a sub-, and supersolution of (E) in $(0, T) \times \mathbf{R}$, respectively. Suppose that $u^{-} \leq u^{+}$in $(0, T) \times \mathbf{R}$ and $\left(u^{+}\right)^{*}<+\infty$ and $\left(u^{-}\right)_{*}>-\infty$ in $[0, T) \times \mathbf{R}$. Suppose that $u^{-}$and $u^{+}$ are periodic in the spatial variable $x$ with period $w$. Then there exists a generalized solution $u$ of $(E)$ such that $u^{-} \leq u \leq u^{+}$in $(0, T) \times \mathbf{R}$ and that $u$ is periodic in $x$ with period $\varpi$.

3.5. Existence Theorem for periodic initial data. Assume conditions (F1) and (F2). Assume (F3) if $F$ depends on $t$. Suppose that $u_{0} \in C(\mathbf{R})$ is periodic with period $\varpi$. Then there exists a unique function $u \in C([0, T) \times \mathbf{R})$ that satisfies

$$
\begin{aligned}
& u \text { is a generalized solution of }(E) \text { in }(0, T) \times \mathbf{R}, \\
& u(0, x)=u_{0}(x) \text { for } x \in \mathbf{R}, \\
& u(t, x+\varpi)=u(t, x) \quad \text { for }(t, x) \in[0, T) \times \mathbf{R} .
\end{aligned}
$$

In particular, if $T$ is arbitrary, then $u$ can be extended globally in time.

3.6. Existence Theorem for the Dirichlet problem. Assume conditions (F1) and (F2). Assume (F3) if $F$ depends on $t$. Let $\Omega$ be a bounded open interval. Assume also that $F(t, p, X)=-F(t, p,-X)$. Suppose that $u_{0} \in C(\bar{\Omega})$ satisfies $u_{0}=0$ on $\partial \Omega$. Then there is a unique generalized solution $u \in C([0, T) \times \bar{\Omega})$ of $(E)$ with $u(0, x)=u_{0}(x)$ and $u=0$ on $\partial \Omega$.

3.7. Remark on the Dirichlet problem. Theorem 3.6 applies to

$$
u_{t}-a\left(u_{w}\right) \Lambda_{W}(u)=0
$$

but it does not apply to

$$
u_{t}-a\left(u_{x}\right)\left(\Lambda_{W}(u)-C\right)=0
$$

even if $C$ is a (nonzero) constant. This is not a technical restriction. In fact, even for smooth $W$ with $W^{\prime \prime}>0$ a local solution to (3.2) may break down at the boundary $\partial \Omega$ in the sense that the gradient on $\partial \Omega$ blows up in a finite time. This phenomena is sometimes called the boundary detachment phenomena (see e.g. [KK]). To solve this problem globally in time we interpret the Dirichlet condition in the viscosity sense as in [KK]. We do 
not pursue this problem in this paper. Our global solution in Theorem 3.6 actually attains zero on $\partial \Omega$ for all time so it should not apply to (3.2).

Theorem 3.6 follows from Theorem 3.5. Indeed, we may assume $\boldsymbol{\Omega}=$ $(0,-\infty / 2)$. We extend initial data $u_{0}$ in $(-\infty / 2,0]$ so that $u_{0}(x)=-u_{0}(-x)$ for $x \in(-\varpi / 2,0)$. We extend $u_{0}$ in $\mathbf{R}$ so that it is periodic in $x$ with period $\varpi$. Since $u_{0}=0$ on $\partial \Omega$, our extended $u_{0}$ is continuous. Applying Theorem 3.5 to get a unique generalized solution $u \in C([0, T) \times \mathbf{R})$ of $(\mathrm{E})$ with $u(0, x)=u_{0}(x)$ and $u(t, x+\infty)=u(t, x)$. By the symmetry assumptions on $F$, we see that $v(t, x)=-u(t,-x)$ solves (E). By Theorem 3.2 we have $u=v$ which implies $u$ is odd in $x$. In particular $u=0$ at $x=0$. A similar argument shows that $u=0$ at $x=\varpi / 2$. We thus observe that $u$ satisfies the Dirichlet condition. Since $u$ solves $(\mathrm{E})$ in $(0, T) \times \mathbf{R}$ it also solves $(\mathrm{E})$ in $(0, T) \times \Omega$ (Proposition 6.19). This property is not trivial and it will be proved in $\S 6$. The proof of Theorem 3.6 is now complete.

3.8. Remark on other boundary value problems. Theorem 3.5 also applies to solve the homogeneous Neumann problem if $F$ and $W$ satisfy

$$
F(t, p, X)=F(t,-p, X), \quad W(p)=W(-p)
$$

provided that we appropriately define generalized solution of (E) with the Neumann condition $u_{s}=0$ on $\partial \Omega$. This assertion applies to both (3.1) and (3.2) provided that $a(p)=a(-p)$ and $W(p)=W(-p)$. We shall discuss the Neumann problem in one of our forthcoming papers.

3.9. Regularity. We wonder whether or not a solution in our Existence Theorem 3.5 is regular if initial data is regular. As observed in $\$ 2$ even if initial data is smooth, the solution may not be $C^{1}$ in space. However, it is Lipschitz in space. A pricise form is presented below. A similar property is proved in $[F G]$ for their solution to (1.1).

3.10. Lipschitz Preserving Theorem. Assume that the same hypotheses of Theorem 3.5 concerning $F$ and $u_{0}$ and let $u$ be the solution with initial data $u_{0}$. Let $\nu_{i}$ be either +1 or -1 for $i=1,2$ and let $L$ be a positive constant. If

$$
\nu_{1} u_{0}(x) \leq \nu_{1} u_{0}\left(x+\nu_{2} h\right)+L|h|
$$

for all $h>0, x \in \mathbf{R}$,

$$
\nu_{1} u(t, x) \leq \nu_{1} u\left(t, x+\nu_{2} h\right)+L|h|
$$

for all $h>0, x \mathbf{R}, 0 \leq t<T$. In particular, if $u_{0}$ is Lipschitz with constant $L$ i.e.

$$
\left|u_{0}(x)-u_{0}(y)\right| \leq L|x-y|
$$


for all $x, y \in \mathbf{R}$, then

$$
|u(t, x)-u(t, y)| \leq L|x-y|
$$

for all $x, y \in \mathbf{R}, 0 \leq t<T$.

Proof. This is an easy corollary of the Comparison Theorem 3.2 since $F$ is independent of $u$ and $x$ so that $u\left(x+\nu_{2} h\right) \pm L|h|$ os a solution of (E) (cf. [GGIS, Corollary 2.11], [G, Theorem 3.6]). If $\nu_{1}=1$, we compare $u$ with $v(t, x)=u\left(t, x+\nu_{2} h\right)+L|h|$. Since both $u$ and $v$ are solution of (E), by Theorem 3.2 we have $u(t, x) \leq v(t, x)$. Similarly if $\nu_{1}=-1$, we compare $u$ with $v(t, x)=u\left(t, x+\nu_{2} h\right)-L|h|$ to get $u \geq v$.

In $\$ 4$ we prepare the key maximum principle. In $\$ 5$ we introdnce convolutions with faceted functions. In $\S 6$ we give several equivalent definitions of solutions. In $\S 7$ we prove Theorems 3.1 and 3.2 based on the results in $\S$ $4-\S 6$. In $\S 8$ we prove Theorems 3.3 and 3.4. In the last section we prove Theorem 3.5 based on Theorems 3.2 and 3.4.

We are forced to assume that uniform continuity (F3) of $F$ if $F$ depends on $t$ in Comparison Theorems. We believe this restriction comes from the method. (In the proof in $\$ 7$ we have no estimates of the length of facets of test functions from below when parameter moves.)

\section{§4. Maximum Principle.}

In this section we derive various maximum principles for faceted functions, which is the key tool to prove our Comparison Theorem.

A classical maximum principle says:

4.1. Proposition (Maximum Principle for $C^{2}$ functions $I$ ). Let $\Omega$ be a domain in $\mathbf{R}^{n}$. If a function $f \in C^{2}(\Omega)$ takes a local maximum over $\Omega$ at $\hat{x}$, then $\nabla f(\hat{x})=0$ and the Hessian Hess $f(\hat{x}) \leq 0$.

As an easy application we get:

4.2. Proposition (Maximum Principle for $C^{2}$ functions II). Let $\Omega$ be an open interval. Let $\hat{z}, \hat{y}$ be in $\Omega$. Suppose that functions $f_{1}, f_{2} \in C^{2}(\Omega)$ and $\theta \in C^{2}(\mathbf{R})$ satisfy

$$
\left\{\begin{array}{l}
f_{1}(x)+f_{2}(y) \leq \theta(x-y) \\
f_{1}(\hat{x})+f_{2}(\hat{y})=\theta(\hat{x}-\hat{y})
\end{array} \quad \text { for all } x \text { and } y \in \Omega\right.
$$

Then

$$
f_{1}^{\prime \prime}(\hat{x})+f_{2}^{\prime \prime}(\hat{y}) \leq 0 .
$$

This type of maximum principle for semicontinuous functions is a key tool to establish comparison theorem for viscosity solutions of degenerate elliptic and parabolic PDE's [CIL]; see $\$ \mathrm{G}$ in $\$ 7$.

We extend this type of the maximum principle for faceted functions. 
4.3. Theorem (Maximum Principle for faceted functions). Let $\Omega$ be an open interval. Let $f_{1}, f_{2}(\in C(\Omega))$ be faceted at $\hat{x}$, and $\hat{y}$, respectively, with slope 0 in $\Omega$. Suppose that $\hat{x}, \hat{y} \in \Omega$ and $\hat{x}-\hat{y} \in I$, where $I$ is a closed interval containing 0 ( $I$ may be a singleton). Suppose that $\theta_{0} \in C(\mathbf{R})$ satisfies

$$
\begin{cases}\theta_{0}=0 & \text { in } I \\ \theta_{0}>0 & \text { otherwise. }\end{cases}
$$

If functions $f_{1}$ and $f_{2}$ satisfy

$$
\left\{\begin{array}{l}
f_{1}(x)+f_{2}(y) \leq \theta_{0}(x-y) \quad \text { for all } x \text { and } y \in \Omega \\
f_{1}(\hat{x})+f_{2}(\hat{y})=\theta_{0}(\hat{x}-\hat{y})
\end{array}\right.
$$

then

$$
\frac{\chi\left(f_{1}, \hat{x}\right)}{L\left(f_{1}, \hat{x}\right)}+\frac{\chi\left(f_{2}, \hat{y}\right)}{L\left(f_{2}, \hat{y}\right)} \leq 0 .
$$

This is not difficult to prove directly. We give maximum principle for faceted functions depending on time, which generalizes Theorem 4.3; see Remark 4.10. Its corollary will be applied in the proof of Comparison Theorem, where we need to estimate 'time derivative' of functions although they are not differentiable.

4.4. Notation. We use following conventions of notation in this section.

(i) For $\left(\hat{t}_{1}, \hat{x}_{1}\right),\left(\hat{t}_{2}, \hat{x}_{2}\right) \in(0, T) \times \mathbf{R}$, let $u_{j}:(0, T) \times \mathbf{R} \rightarrow \mathbf{R}$ be a uppersemicontinuous function $(j=1,2)$ such that

$$
\left\{\begin{array}{l}
u_{j}\left(t_{j}, \cdot\right) \in C(\mathbf{R}) \text { for each } t_{j} \in(0, T) \\
u_{j}\left(\hat{t}_{j}, \cdot\right) \text { is faceted at } \hat{x}_{j} \text { in } \mathbf{R} \text { with slope } 0 \\
L\left(u_{j}\left(\hat{t}_{j}, \cdot\right), \hat{x}\right)<\infty, \quad \text { for } j=1,2
\end{array}\right.
$$

The faceted region $R\left(u_{j}\left(\hat{t}_{j}, \cdot\right), \hat{x}_{j}\right)$ is denoted by $\left[a_{j}, b_{j}\right], j=1,2$.

(ii) Let $\theta:[0, \infty) \rightarrow[0, \infty)$ be a continuous function such that

$$
\begin{cases}\theta=0 \text { in }[0, \sigma] & \text { with some } \sigma>0 \\ \theta>0 & \text { otherwise. }\end{cases}
$$

(iii) $S \in C((0, T) \times(0, T))$.

(iv) $\Theta\left(t_{1}, x_{1}, t_{2}, x_{2}\right)=u_{1}\left(t_{1}, x_{1}\right)+u_{2}\left(t_{2}, x_{2}\right)-\theta\left(\left|x_{1}-x_{2}-\hat{q}\right|\right)-S\left(t_{1}, t_{2}\right)$ with $\hat{\boldsymbol{q}}=\hat{\boldsymbol{x}}_{1}-\hat{\boldsymbol{x}}_{2}$.

(v) For $j=1,2$ we set $j^{\prime}=\{1,2\} \backslash\{j\}$. 
4.5. Theorem (Maximum Principle for faceted functions with the time direction). Suppose that $\left(\hat{t}_{1}, \hat{\boldsymbol{x}}_{1}, \hat{t}_{2}, \hat{\boldsymbol{x}}_{2}\right) \in(0, T) \times \mathbf{R} \times(0, T) \times \mathbf{R}$ is a maximizer of $\Theta$ over $(0, T) \times \mathbf{R} \times(0, T) \times \mathbf{R}$, with $\hat{q}=\hat{x}_{1}-\hat{x}_{2}$.

(i) It holds that

$$
\frac{\chi\left(u_{1}\left(\hat{t}_{1}, \cdot\right), \hat{x}_{1}\right)}{L\left(u_{1}\left(\hat{t}_{1}, \cdot\right), \hat{x}_{1}\right)}+\frac{\chi\left(u_{2}\left(\hat{t}_{2}, \cdot\right), \hat{x}_{2}\right)}{L\left(u_{2}\left(\hat{t}_{2}, \cdot\right), \hat{x}_{2}\right)} \leq 0 .
$$

(ii) For $j=1,2$, let $\left(I_{j}\right)$ denote the inequality

$$
u_{j}\left(t_{j}, x_{j}\right)-u_{j}\left(\hat{t}_{j}, \hat{x}_{j}\right) \leq\left. S\left(t_{1}, t_{2}\right)\right|_{t_{j^{\prime}}=\hat{t}_{j^{\prime}}}-S\left(\hat{t}_{1}, \hat{t}_{2}\right) .
$$

Then

$\left(I_{1}\right)$ holds for all $(0, T) \times\left[a_{2}+\hat{q}, b_{2}+\hat{q}\right]$,

$\left(I_{2}\right)$ holds for all $(0, T) \times\left[a_{1}-\hat{q}, b_{1}-\hat{q}\right]$.

Moreover if $\tilde{a}_{j} \geq \tilde{a}_{j^{\prime}}$, then $\chi-\left(u_{j}\left(\hat{t}_{j}, \cdot\right), \hat{x}_{j}\right)=-1$ and there exists $\delta>0$ such that

$\left(I_{j}\right)$ holds for all $(0, T) \times\left[a_{j}-\delta, a_{j}\right)$,

and if $\tilde{b}_{j} \leq \tilde{b}_{j^{\prime}}$, then $\chi_{+}\left(u_{j}\left(\hat{t}_{j}, \cdot\right), \hat{x}_{j}\right)=-1$ and there exists $\delta>0$ such that

$$
\left(I_{j}\right) \text { holds for all }(0, T) \times\left(b_{j}, b_{j}+\delta\right] \text {, }
$$

where

$$
\left[\tilde{a}_{1}, \tilde{b}_{1}\right]=\left[a_{1}, b_{1}\right], \quad\left[\tilde{a}_{2}, \tilde{b}_{2}\right]=\left[a_{2}+\hat{q}, b_{2}+\hat{q}\right] .
$$

4.6. Corollary. Assume the same hypotheses of Theorem 4.5. Let $\Omega$ be an open interval. Suppose that $\left[\hat{a}_{1}, \hat{b}_{1}\right],\left[\hat{a}_{2}, \hat{b}_{2}\right] \subset \Omega$, and $\hat{x}_{j} \in\left[\hat{a}_{j}, \hat{b}_{j}\right]$ for $j=1,2$, where

$$
\left[\hat{a}_{1}, \hat{b}_{1}\right]=\left[a_{1}, b_{1}\right] \cap\left[a_{2}+\hat{q}, b_{2}+\hat{q}\right], \quad\left[\hat{a}_{2}, \hat{b}_{2}\right]=\left[a_{1}-\hat{q}, b_{1}-\hat{q}\right] \cap\left[a_{2}, b_{2}\right] .
$$

Then there exist uppersemicontinuous functions $v_{1}$ and $v_{2}:(0, T) \times \Omega \rightarrow \mathbf{R}$ such that

$$
\begin{aligned}
& \left\{\begin{array}{l}
v_{j}\left(t_{j}, \cdot\right) \in C(\Omega) \text { for each } t_{j} \in(0, T), \\
v_{j}\left(\hat{t}_{j}, \cdot\right) \text { is faceted at } \hat{x}_{j} \text { in } \Omega \text { with slope } 0 \text { in } \Omega, \\
u_{j} \leq v_{j} \text { in }(0, T) \times \Omega, \\
v_{j}\left(\hat{t}_{j}, \hat{x}_{j}\right)=u_{j}\left(\hat{t}_{j}, \hat{x}_{j}\right), \quad \text { for } j=1,2,
\end{array}\right. \\
& \left\{\begin{array}{l}
R\left(v_{1}\left(\hat{t}_{1}, \cdot\right), \hat{x}_{1}\right)=\left[\hat{a}_{1}, \hat{b}_{1}\right] \quad R\left(v_{2}\left(\hat{t}_{2}, \cdot\right), \hat{x}_{2}\right)=\left[\hat{a}_{2}, \hat{b}_{2}\right], \\
L\left(v_{1}\left(\hat{t}_{1}, \cdot\right), \hat{x}_{1}\right)=L\left(v_{2}\left(\hat{t}_{2}, \cdot\right), \hat{x}_{2}\right),
\end{array}\right. \\
& \chi\left(v_{1}\left(\hat{t}_{1}, \cdot\right), \hat{x}_{1}\right)+\chi\left(v_{2}\left(\hat{t}_{2}, \cdot\right), \hat{x}_{2}\right) \leq 0 .
\end{aligned}
$$


(iv) Let $\left(I_{j}^{\prime}\right)$ denote the inequality

$$
v_{j}\left(t_{j}, x_{j}\right)-v_{j}\left(\hat{t}_{j}, \hat{x}_{j}\right) \leq\left. S\left(t_{1}, t_{2}\right)\right|_{t_{j^{\prime}}=\hat{t}_{j^{\prime}}}-S\left(\hat{t}_{1}, \hat{t}_{2}\right)
$$

Then

$$
\left(I_{j}^{\prime}\right) \text { holds for all }(0, T) \times\left[\hat{a}_{j}, \hat{b}_{j}\right] \text { for } j=1,2 \text {. }
$$

Moreover if $\chi-\left(v_{j}\left(\hat{t}_{j}, \cdot\right), \hat{x}_{j}\right)=-1$ then there exists $\delta>0$ such that

$$
\left(I_{j}^{\prime}\right) \text { holds for all }(0, T) \times\left[\hat{a}_{j}-\delta, \hat{a}_{j}\right)
$$

and if $\chi_{+}\left(v_{j}\left(\hat{t}_{j}, \cdot\right), \hat{x}_{j}\right)=-1$ then there exists $\delta>0$ such that

$$
\left(I_{j}^{\prime}\right) \text { holds for all }(0, T) \times\left(\hat{b}_{j}, \hat{b}_{j}+\delta\right] \text {. }
$$

We shall prove Theorem 4.5 and Corollary 4.6 in several steps.

4.7. Lemma. Assume the same hypotheses of Theorem 4.5. Then

$$
\begin{aligned}
& \left(I_{1}\right) \text { holds for all }\left(t_{1}, x_{1}\right) \in(0, T) \times\left[a_{2}+\hat{q}, b_{2}+\hat{q}\right] \text {. } \\
& \left(I_{2}\right) \text { holds for all }\left(t_{2}, x_{2}\right) \in(0, T) \times\left[a_{1}-\hat{q}, b_{1}-\hat{q}\right] \text {. }
\end{aligned}
$$

Proof. By assumptions of the theorem, we have (4.4)

$$
\begin{aligned}
& u_{1}\left(t_{1}, x_{1}\right)+u_{2}\left(t_{2}, x_{2}\right)-\theta\left(\left|x_{1}-x_{2}-\hat{q}\right|\right)-S\left(t_{1}, t_{2}\right) \\
& \leq u_{1}\left(\hat{t}_{1}, \hat{x}_{1}\right)+u_{2}\left(\hat{t}_{2}, \hat{x}_{2}\right)-S\left(\hat{t}_{1}, \hat{t}_{2}\right) \quad \text { for }\left(t_{1}, x_{1}\right),\left(t_{2}, x_{2}\right) \in(0, T) \times \mathbf{R} .
\end{aligned}
$$

We take $t_{2}=\hat{t}_{2}$ and $x_{2}=x_{1}-\hat{q}$ for $x_{1} \in\left[a_{2}+\hat{q}, b_{2}+\hat{q}\right]$ in (4.4) to get

$$
\begin{aligned}
u_{1}\left(t_{1}, x_{1}\right)+u_{2}\left(\hat{t}_{2}, x_{1}-\hat{q}\right)-S\left(t_{1}, \hat{t}_{2}\right) & \leq u_{1}\left(\hat{t}_{1}, \hat{x}_{1}\right)+u_{2}\left(\hat{t}_{2}, \hat{x}_{2}\right)-S\left(\hat{t}_{1}, \hat{t}_{2}\right) \\
& \text { for }\left(t_{1}, x_{1}\right) \in(0, T) \times\left[a_{2}+\hat{q}, b_{2}+\hat{q}\right] .
\end{aligned}
$$

Since $x_{1}-\hat{q} \in\left[a_{2}, b_{2}\right]$, we have $u_{2}\left(\hat{t}_{2}, x_{1}-\hat{q}\right)=u_{2}\left(\hat{t}_{2}, \hat{x}_{2}\right)$, which implies $(\mathrm{i})$. Similarly, we get (ii) by plugging $t_{1}=\hat{t}_{1}, x_{1}=x_{2}+\hat{q}$ for $x_{2} \in R\left(u_{1}\left(\hat{t}_{1}, \cdot\right), \hat{x}_{1}\right)-$ $\hat{q}$.

4.8. Lemma. Assume the same hypotheses of Theorem 4.5.

(i) If $\tilde{a}_{j}>\tilde{a}_{j^{\prime}}$, then $\chi-\left(u_{j}\left(\hat{t}_{j}, \cdot\right), \hat{x}_{j}\right)=-1$ and there exists $\delta>0$ such that

$\left(I_{j}\right)$ holds for all $\left(t_{j}, x_{j}\right) \in(0, T) \times\left[a_{j}-\delta, a_{j}\right)$. 
(ii) If $\tilde{b}_{j}<\tilde{b}_{j^{\prime}}$, then $\chi_{+}\left(u_{j}\left(\hat{t}_{j}, \cdot\right), \hat{x}_{j}\right)=-1$ and there exists $\delta>0$ such that $\left(I_{j}\right)$ holds for all $\left(t_{j}, x_{j}\right) \in(0, T) \times\left(b_{j}, b_{j}+\delta\right]$.

Proof. We only prove case (i), since the proof of (ii) is similar. By Lemma 4.7,

$\left(I_{j}\right)$ holds for all $\left(t_{j}, x_{j}\right) \in(0, T) \times\left[a_{j^{\prime}}-(-1)^{j} \hat{q}, b_{j},-(-1)^{j} \hat{q}\right]$, for $j=1,2$.

Since $\left[a_{j^{\prime}}-(-1)^{j} \hat{q}_{,} b_{j^{\prime}}-(-1)^{j} \hat{q}\right] \supset\left[a_{j^{\prime}}-(-1)^{j} \hat{q}, a_{j}\right)=\left[a_{j}-\delta, a_{j}\right)$ with $\delta=\tilde{a}_{j}-\tilde{a}_{j^{\prime}}$,

$\left(I_{j}\right)$ holds for all $\left(t_{j}, x_{j}\right) \in(0, T) \times\left[a_{j}-\delta, a_{j}\right)$.

It remains to prove that $\chi-\left(u_{j}\left(\hat{t}_{j}, \cdot\right), \hat{x}_{j}\right)=-1$. Plugging $t_{j}=\hat{t}_{j}$ into $\left(I_{j}\right)$, we have

$$
u_{j}\left(\hat{t}_{j}, x_{j}\right) \leq u_{j}\left(\hat{t}_{j}, \hat{x}_{j}\right) \text { for all } x_{j} \in\left[a_{j}-\delta, a_{j}\right) .
$$

Since $R\left(u_{j}\left(\hat{t}_{j}, \cdot\right), \hat{x}_{j}\right)=\left[a_{j}, b_{j}\right]$, there exists $\eta>0$ such that

$$
u_{j}\left(\hat{t}_{j}, x_{j}\right)<u_{j}\left(\hat{t}_{j}, \hat{x}_{j}\right) \text { for all } x_{j} \in\left[a_{j}-\eta, a_{j}\right),
$$

which implies $\chi-\left(u_{j}\left(\hat{t}_{j}, \cdot\right), \hat{x}_{j}\right)=-1$.

4.8. Lemma. Assume the same hypotheses of Theorem 4.5. Let $\sigma>0$ be as in $\$ 4.4$ (ii).

(i) Suppose $\tilde{a}_{1}=\tilde{a}_{2}$, then $\chi-\left(u_{j}\left(\hat{t}_{j}, \cdot\right), \hat{x}_{j}\right)=-1$ and

$\left(I_{j}\right)$ holds for all $\left(t_{j}, x_{j}\right) \in(0, T) \times\left[a_{j}-\sigma, a_{j}\right) \quad$ for $j=1,2$.

(ii) Suppose $\tilde{b}_{1}=\tilde{b}_{2}$, then $\chi_{+}\left(u_{j}\left(\hat{t}_{j}, \cdot\right), \hat{x}_{j}\right)=-1$ holds and

$$
\left(I_{j}\right) \text { holds for all }\left(t_{j}, x_{j}\right) \in(0, T) \times\left(b_{j}, b_{j}+\sigma\right] \quad \text { for } j=1,2 \text {. }
$$

Proof. We only give the proof of (i) here, since the proof of (ii) is parallel. By assumption of Theorem 4.5, we have inequality (4.4). Plugging $t_{j^{\prime}}=\hat{t}_{j^{\prime}}$ and $x_{j^{\prime}}=a_{j^{\prime}}$ in (4.4), we have

$$
\begin{gathered}
u_{j}\left(t_{j}, x_{j}\right)-\theta\left(\left|x_{j}-a_{j}\right|\right)-\left.S\left(t_{1}, t_{2}\right)\right|_{t_{j^{\prime}}=\hat{t}_{j^{\prime}}} \leq u_{j}\left(\hat{t}_{j}, \hat{x}_{j}\right)-S\left(\hat{t}_{1}, \hat{t}_{2}\right) \\
\text { for }\left(t_{j}, x_{j}\right) \in(0, T) \times \mathbf{R},
\end{gathered}
$$

since $\tilde{a}_{1}=\tilde{a}_{2}$. For $x_{j} \in\left[a_{j}-\sigma, a_{j}+\sigma\right]$, we have $\theta\left(\left|x_{j}-a_{j}\right|\right)=0$, which implies

$$
\left(I_{j}\right) \text { holds for all }\left(t_{j}, x_{j}\right) \in(0, T) \times\left[a_{j}-\sigma, a_{j}\right) \text {. }
$$


Plugging $t_{j}=\hat{t}_{j}$ in $\left(I_{j}\right)$, we have

$$
u_{j}\left(\hat{t}_{j}, x_{j}\right) \leq u_{j}\left(\hat{t}_{j}, \hat{x}_{j}\right) \text { for all } x_{j} \in\left[a_{j}-\sigma, a_{j}\right)
$$

which implies $\chi_{-}\left(u_{j}\left(t_{j}, \cdot\right), x_{j}\right)=-1$.

Proof of Theorem 1.5. By Lemmas 4.8 and 4.9, there exist $j$ and $k \in\{1,2\}$ such that

$$
\chi_{-}\left(u_{j}\left(\hat{t}_{j}, \cdot\right), \hat{x}_{j}\right)=-1 \text { and } \chi_{+}\left(u_{k}\left(\hat{t}_{k}, \cdot\right), \hat{x}_{h}\right)=-1
$$

which implies

$$
\chi\left(u_{1}\left(\hat{t}_{1}, \cdot\right), \hat{x}_{1}\right)+\chi\left(u_{2}\left(\hat{t}_{2}, \cdot\right), \hat{x}_{2}\right) \leq 0 .
$$

For $\left(\chi\left(u_{1}\left(\hat{t}_{1}, \cdot\right), \hat{x}_{1}\right), \chi\left(u_{2}\left(\hat{t}_{2}, \cdot\right), \hat{x}_{2}\right)\right)=(-1,-1),(-1,0),(0,0)$ and $(0,-1)$, validity of inequality in (i) is trivial. We shall check (i) when

$$
\left(\chi\left(u_{1}\left(\hat{t}_{1}, \cdot\right), \hat{x}_{1}\right), \chi\left(u_{2}\left(\hat{t}_{2}, \cdot\right), \hat{x}_{2}\right)\right)=(-1,1) .
$$

Since Lemmas 4.8 and 4.9 imply

$$
R\left(u_{1}\left(\hat{t}_{1}, \cdot\right), \hat{x}_{1}\right) \subset R\left(u_{2}\left(\hat{t}_{2}, \cdot\right), \hat{x}_{2}\right)+\hat{q}
$$

we have

$$
L\left(u_{1}\left(\hat{t}_{1}, \cdot\right), \hat{x}_{1}\right) \geq L\left(u_{2}\left(\hat{t}_{2}, \cdot\right), \hat{x}_{2}\right),
$$

which implies (i). When $\left(\chi\left(u_{1}\left(\hat{t}_{1}, \cdot\right), \hat{x}_{1}\right), \chi\left(u_{2}\left(\hat{t}_{2}, \cdot\right), \hat{x}_{2}\right)\right)=(1,-1)$, we can prove similarly.

The property (ii) is given by Lemmas $4.7,4.8$ and 4.9.

4.10. Remark. Even if $\sigma=0$ in Notation 4.4(ii) assertions of Lemma 4.7 and 4.8 are still valid. If $\tilde{a}_{1}=\tilde{a}_{2}$, then setting $t_{j}=\hat{t}_{j}, x_{2}=x_{1}-\hat{q}$ in (4.4) we have

$$
\chi-\left(u_{j}\left(\hat{t}_{j}, \cdot\right), \hat{z}\right)=-1
$$

for $j=1$ or 2 . (However, the assertion for $\left(I_{j}\right)$ in Lemma 4.9 may not be true.)

We observe that the proof of Theorem 4.5(i) is still valid even if $\sigma=0$. By this remark, we note that Theorem 4.3 follows from Theorem 4.5(i) by setting

$$
u_{j}(t, x)=f_{j}(x), \quad S\left(t_{1}, t_{2}\right)=0, \quad \hat{x}_{1}=\hat{x}, \quad \hat{x}_{2}=\hat{y}
$$

with a choice of $\theta$ in Notation 4.4(ii) such that

$$
\theta(|z-\hat{q}|) \geq \theta_{0}(z), \quad \text { for } z \in \mathbf{R} .
$$


Proof of Corollary 4.6. We use the notation of Theorem 4.5. Define functions $f_{j,+}$ and $f_{j,-} \in C(\Omega)$ for $j=1,2$ satisfying (A) and (B).

(A) If $\tilde{a}_{j^{\prime}}=\tilde{a}_{j}$ then $f_{j,-} \equiv 0 \equiv f_{j^{\prime},-}$ in $\Omega$, and if $\tilde{a}_{j^{\prime}}<\tilde{a}_{j}$ then

$$
f_{j,-} \equiv 0 \text { in } \Omega \quad \text { and } \quad\left\{\begin{array}{l}
f_{j^{\prime},-}>0 \text { in }\left(\tilde{a}_{j^{\prime}}, \tilde{a}_{j}\right), \\
f_{j^{\prime},-}=0 \text { in } \Omega \backslash\left(\tilde{a}_{j^{\prime}}, \tilde{a}_{j}\right) .
\end{array}\right.
$$

(B) If $\tilde{b}_{j}=\tilde{b}_{j}$, then $f_{j,+} \equiv 0 \equiv f_{j,+}$ in $\Omega$, and if $\tilde{b}_{j}<\tilde{b}_{j,}$ then

$$
f_{j,+} \equiv 0 \text { in } \Omega \quad \text { and } \quad\left\{\begin{array}{l}
f_{j^{\prime},+}>0 \text { in }\left(\tilde{b}_{j}, \tilde{b}_{j^{\prime}}\right), \\
f_{j^{\prime},+}=0 \text { in } \Omega \backslash\left(\tilde{b}_{j^{\prime}}, \tilde{b}_{j^{\prime}}\right) .
\end{array}\right.
$$

\section{Setting}

$$
v_{j}(t, x)=u_{j}(t, x)+f_{j,-}(x)+f_{j,+}(x) \quad \text { for }(t, x) \in Q \text { and } i=1,2,
$$

we see (i), (ii) and (iii) hold.

Since $\left[\hat{a}_{j}, \hat{b}_{j}\right] \subset\left[a_{j^{\prime}}-(-1)^{j} \hat{q}, b_{j},-(-1)^{j} \hat{q}\right]$, we get $(4.1)$ by Theorem 4.5 (ii). By the definition of $v_{1}$ and $v_{2}$,

$$
\begin{cases}\tilde{a}_{j^{\prime}} \leq \tilde{a}_{j} & \text { if } \chi-\left(v_{j}\left(\hat{t}_{j}, \cdot\right), \hat{x}_{j}\right)=-1 \\ \tilde{b}_{j} \leq \tilde{b}_{j^{\prime}} & \text { if } \chi_{+}\left(v_{j}\left(\hat{t}_{j}, \cdot\right), \hat{x}_{j}\right)=1\end{cases}
$$

which implies (4.2) and (4.3).

\section{§5. Convolutions.}

To regularize semicontinuous functions it is convenient to use sup- and inf- convolutions of functions. A typical way to regularize is to consider a sup-convolution

$$
f(x, \lambda)=\sup _{\xi \in \mathbb{R}}\left\{f(\xi)-|x-\xi|^{2} / \lambda\right\}
$$

for an (uppersemicontinuous) function $f$, where $\lambda$ is a small positive parameter [CIL]. However, this type of convolution is not convenient for our purpose. We consider a sup-convolution with a faceted function replacing $|x-\xi|^{2} / \lambda$. The goal of this section is to study such sup-convolutions. A new feature of our sup-convolution is that if $f$ takes a local maximum at $\hat{x}$ then its sup-convolution is faceted near $\hat{\boldsymbol{x}}$.

Let $\phi$ be a function from $\mathbf{R} \times(0,1]$ to $[0, \infty)$. We often assume the following conditions on $\phi$.

(Ф0) For each $\lambda, 0<\lambda \leq 1, \phi(\cdot, \lambda)$ is Lipschitz on every bounded set in R. 
( 11$) \quad \phi(\xi, \lambda)$ is even in $\xi$, i.e., $\phi(\xi, \lambda)=\phi(-\xi, \lambda)$.

(\$2) $\quad \phi(\xi, \lambda)$ is nonincreasing in $\lambda$ for all $\xi$.

(\$3) $\lim _{\xi \rightarrow \infty} \phi(\xi, 1)=\infty$ and $\phi(\xi, \lambda)$ is nondecreasing in $\xi \geq 0$, for $0<\lambda \leq$

1.

(\$4) $\quad \lim _{\lambda \downarrow 0} \phi(\xi, \lambda)=\infty$ unless $\xi \neq 0$ and $\phi(0, \lambda)=0,0<\lambda \leq 1$.

(Ф5) Let $\sigma_{\lambda}=\sup \{|\xi| ; \phi(\xi, \lambda)=0\}$. Then for $0<\lambda \leq 1, \sigma_{\lambda}>0\left(\sigma_{\lambda}\right.$ is nondecreasing in $\lambda$ and $\lim _{\lambda \downarrow 0} \sigma_{\lambda}=0$ if we assume $(\Phi 2)$ and $\left.(\Phi 4)\right)$.

Let $f$ be a function on $\mathbf{R}$ with values in $[-\infty, \infty)$. Then

$$
f^{\lambda}(x)=\sup _{\xi \in \mathbf{R}}\{f(\xi)-\phi(\xi-x, \lambda)\}
$$

is called a sup-convolution of $f$ by $\phi$. Our assumptions in ( $\Phi 0)-(\Phi 4)$ are rather standard. For example

$$
\phi(x, \lambda)=|x|^{2} / \lambda
$$

satisfies $(\Phi 0)-(\Phi 4)$. However it does not satisfy $(\Phi 5)$, where $\phi(\xi, \lambda)=0$ is assumed to be faceted at $\xi=0$ with slope zero. Instead of this choice of $\phi$. we often use

$$
\vartheta(x, \lambda)=\lambda \bar{\vartheta}(x / \lambda)
$$

with

$$
\bar{\vartheta}(x)=\left\{\begin{array}{lll}
(x-1)^{2} & \text { for } & x>1 \\
0 & \text { for } & |x|<1 \\
(x+1)^{2} & \text { for } & x<-1
\end{array}\right.
$$

Clearly, $(\Phi 0)-(\Phi 5)$ are fulfilled for $\phi=\vartheta$. We recall fundamental properties of $f^{\lambda}$ in (5.1) which are familiar when $\phi=|x|^{2} / \lambda[L L]$. If $\phi=|x|^{2} / \lambda, f^{\lambda}$ is often used in functional analysis and is called the Yosida approximation of $f$. Another choice of $\phi$ (which does not satisfy ( $\Phi 5)$ ) was used in [IR] to study singular Hamilton-Jacobi equations.

5.1. Lemma. Assume ( $\Phi 0)-(\Phi 4)$ for $\phi$. Let $f(\not \equiv-\infty)$ be a function on $\mathbf{R}$ with values in $[-\infty, \infty)$ and assume that $f$ is bounded from above on every bounded set in $\mathbf{R}$ and that *

$$
\lim _{|\xi| \rightarrow \infty} \max (f(\xi), 0) / \phi(\xi-x, 1)=0 \quad \text { for each } \quad x \in \mathbf{R}
$$

Let $f^{\lambda}$ be a sup-convolution of $f$ by $\phi$. Then

(i) $f^{\lambda}$ is Lipschitz on every bounded set in $\mathbf{R}$. 
(ii) $f^{\lambda} \geq f^{\mu} \geq f$ for $\lambda \geq \mu>0$ and $\lim _{\lambda \downarrow 0} f^{\lambda}(x)=f^{*}(x)$ for each $x \in \mathbf{R}$.

(iii) Let $B$ and $B^{\prime}$ be bounded open sets in $\mathrm{R}$ with $\bar{B} \subset B^{\prime}$. Then for each $K_{0}>0$ there is $\lambda_{0}\left(K_{0}\right)>0$ such that

$$
\sup _{\boldsymbol{x} \in \bar{B} \bar{B} \xi \notin B^{\prime}} H(\xi, x, \lambda)<-K_{0} \text { for } \lambda<\lambda_{0}\left(K_{0}\right)
$$

with $H(\xi, x, \lambda)=f(\xi)-\phi(\xi-x, \lambda)$.

(a) If $\inf _{\bar{B}} f^{*}>-\infty$, then for $\lambda<\lambda_{0}^{\prime} \equiv \lambda_{0}\left(\max \left(0,-\inf _{\bar{B}} f^{*}\right)\right)$ we have

$$
f^{\lambda}(x)=\sup _{\xi \in B^{\prime}} H(\xi, x, \lambda) \quad \text { for } \quad x \in \bar{B} \text {. }
$$

(b) If $\hat{x}$ is a maximizer of $f$ over $B^{\prime}$, i.e. $f(\hat{x})=\max _{B^{\prime}} f$, then $f^{\lambda}(x) \leq$ $f(\hat{x})$ for $x \in \bar{B}$ provided that

$$
\lambda<\lambda_{0}^{\prime \prime} \equiv \lambda_{0}(\max (0,-f(\hat{x}))) .
$$

Proof. The proof is standard. We give it for completeness.

We may assume that $f$ is uppersemicontinuous by replacing $f$ by $f^{*}$ in (5.1) since the value $f^{\lambda}(x)$ in (5.1) is unchanged with this replacement. have

By (\$2) we have $f^{\lambda}(x) \geq f^{\mu}(x)$ for $\lambda \geq \mu$. By $\phi(0, \lambda)=0$ of $(\Phi 4)$ we

$$
f^{\lambda}(x) \geq f(x)-\phi(x-x, \lambda)=f(x) .
$$

Let $\rho>0$ be any number with

$$
\rho>\bar{\rho}=\sup \{\sigma ; f(x) \equiv-\infty \quad \text { for } \quad|x|<\sigma\} .
$$

Then there is $\rho^{\prime}>\rho$ such that

$$
f^{\lambda}(x)=\sup _{|\xi| \leq \rho^{\prime}} H(\xi, x, \lambda)=\sup _{|\xi| \leq \rho^{\prime}}\{f(\xi)-\phi(\xi-x, \lambda)\},|x| \leq \rho 0<\lambda \leq 1 .
$$

Indeed, by (5.3) there is $\rho_{0}>\rho$ such that

$$
f(\xi) \leq \frac{1}{2} \phi(\xi-x, 1) \quad \text { for } \quad|\xi| \geq \rho_{0},|x| \leq \rho
$$

so that if $|x| \leq \rho$ then

$$
f(\xi)-\phi(\xi-x, \lambda) \leq \frac{1}{2} \phi(\xi-x, 1)-\phi(\xi-x, 1)=-\frac{1}{2} \phi(\xi-x, 1)
$$


by (Ф2). Since there is $x^{\prime},\left|x^{\prime}\right| \leq \rho$ such that $f\left(x^{\prime}\right)>-\infty$,

$$
\begin{aligned}
f^{\lambda}(x) & \geq f\left(x^{\prime}\right)-\phi\left(x^{\prime}-x, \lambda\right) \\
& \geq f\left(x^{\prime}\right)-\sup _{|y| \leq \rho} \phi\left(x^{\prime}-y, \lambda\right)=M \text { for }|x| \leq \rho
\end{aligned}
$$

with some constant $M$ independent of $x$. By (Ф3) one can take $\rho^{\prime}>\rho_{0}$ so large that

$$
-\frac{1}{2} \phi(\xi-x, 1)<M \text { for }|\xi| \geq \rho^{\prime}, \rho \geq|x| .
$$

By (5.5) and (5.6) this implies

$$
f^{\lambda}(x)>f(\xi)-\phi(\xi-x, \lambda) \text { for }|\xi| \geq \rho^{\prime} \text { and } \rho \geq|x| .
$$

We have thus proved (5.4).

Since $f$ is bounded from above on $|x| \leq \rho^{\prime}$, the supremum of (5.4) is finite, so that $f^{\lambda}(x)$ is finite. For each $\rho>\bar{\rho}$ we shall prove that $f^{\lambda}$ is Lipschitz in $|x| \leq \rho$. By (5.4) for each $\epsilon>0$ there is $\xi_{0},\left|\xi_{0}\right| \leq \rho^{\prime}$ such that

$$
f^{\lambda}(x) \leq f\left(\xi_{0}\right)-\phi\left(\xi_{0}-x, \lambda\right)+\epsilon
$$

For $y,|y| \leq \rho$ we have

$$
\begin{aligned}
f^{\lambda}(x) & -f^{\lambda}(y) \leq f\left(\xi_{0}\right)-\phi\left(\xi_{0}-x, \lambda\right)+\epsilon-\left\{f\left(\xi_{0}\right)-\phi\left(\xi_{0}-y, \lambda\right)\right\} \\
& \leq \phi\left(\xi_{0}-y, \lambda\right)-\phi\left(\xi_{0}-x, \lambda\right)+\epsilon
\end{aligned}
$$

Since $\phi(\xi, \lambda)$ is Lipschitz for $|\xi| \leq \rho+\rho^{\prime}$ by $(\Phi 0)$, we have

$$
f^{\lambda}(x)-f^{\lambda}(y) \leq L|x-y|+\epsilon
$$

with some $L>0$. Sending $\epsilon \downarrow 0$ and interchanging the role of $x, y$ we have

$$
\left|f^{\lambda}(x)-f^{\lambda}(y)\right| \leq L|x-y| \quad \text { for } \quad|x| \leq \rho,|y| \leq \rho .
$$

We shall show that

$$
\lim _{\lambda \perp 0} f^{\lambda}(x)=f(x)
$$

Since $f$ is uppersemicontinuous, for each $\epsilon>0$ there is $\delta$ such that

$$
f(\xi) \leq f(x)+\epsilon \text { for }|\xi-x| \leq \delta .
$$




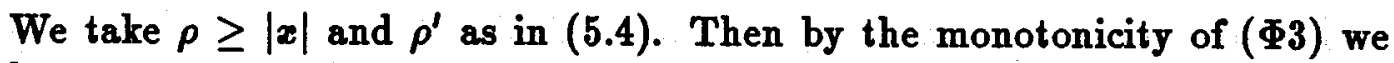
have

$$
\begin{aligned}
& \sup \left\{f(\xi)-\phi(\xi-x, \lambda) ;|\xi| \leq \rho^{\prime},|\xi-x| \geq \delta\right\} \\
& \quad \leq \sup _{|\xi| \leq \rho^{\prime}} f(\xi)-\phi(\delta, \lambda) .
\end{aligned}
$$

By (\$4) for each $K>0$ there is small $\lambda_{0}>0$ such that the right hand side of (5.9) is dominated by $-K$ for $|\lambda| \leq \lambda_{0}$. Applying (5.8) and (5.9) to (5.4) yields

$$
\begin{aligned}
f^{\lambda}(x) & \leq \max \left(\sup _{|\xi-x| \leq \delta}(f(\xi)-\phi(\xi-x, \lambda)),-K\right) \\
& \leq \max (f(x)+\epsilon,-K)
\end{aligned}
$$

if $\lambda<\lambda_{0}$. Take $K$ large such that $-K<f(x)$ and fix $\lambda_{0}$. Then $f^{\lambda}(x) \leq$ $f(x)+\epsilon$ for $\lambda<\lambda_{0}$. Since $f \leq f^{\lambda}$, this implies (5.7).

It remains to prove (iii). We take $\rho(>\bar{\rho})$ such that $\{x \in \mathbf{R} ;|x| \leq \rho\}$ contains $B$. By the proof of (5.4) it suffices to prove that for $\rho^{\prime}>\rho$

$$
\sup _{\approx \in \bar{B}} \sup \left\{H(\xi, x, \lambda) ;|\xi| \leq \rho^{\prime}, \xi \notin B^{\prime}\right\} \leq-K_{0}
$$

for sufficiently small $\lambda, \lambda<\lambda_{0}\left(K_{0}\right)(\leq 1)$. Let $d>0$ be the distance from $\bar{B}$ to $\partial B^{\prime}$. Then by $(\Phi 3)$ with $(\Phi 1)$ we have

$$
H(\xi, x, \lambda)=f(\xi)-\phi(\xi-x, \lambda) \leq f(\xi)-\phi(d, \lambda) \text { for } \xi \notin B^{\prime}, x \in \bar{B} .
$$

Since $f$ is bounded from above on $\left\{\xi \in R ;|\xi| \leq \rho^{\prime}\right\}$, from $(\Phi 4)$ it now follows (5.10) with $\lambda<\lambda_{0}\left(K_{0}\right)$ provided that $\lambda_{0}\left(K_{0}\right)$ is taken so that

$$
\sup _{|\xi| \leq \rho^{\prime}} f(\xi)+K_{0}<\phi\left(d, \lambda_{0}\left(K_{0}\right)\right)
$$

If $K_{0}=\max \left(-\inf _{\bar{B}} f, 0\right)$, then $f^{\lambda}(x) \geq f(x)>-K_{0}$ for $x \in \bar{B}$. If $\lambda<\lambda_{0}^{\prime}=$ $\lambda_{0}\left(K_{0}\right)$ with this $K_{0}$, then $(5.10)$ yields

$$
f^{\lambda}(x)>\sup \left\{H(\xi, x, \lambda), \xi \notin B^{\prime},|\xi| \leq \rho^{\prime}\right\} \quad \text { for } \quad x \in \bar{B},
$$

so that

$$
\begin{aligned}
f^{\lambda}(x) & =\sup \left\{H(\xi, x, \lambda) ;|\xi| \leq \rho^{\prime}\right\} \quad \text { by }(5.4) \\
& =\sup \left\{H(\xi, x, \lambda) ;|\xi| \leq \rho^{\prime}, \xi \in B\right\} \\
& =\sup \{H(\xi, x, \lambda) ; \xi \in B\} \quad \text { for } \quad x \in \bar{B}
\end{aligned}
$$


This completes the proof of $(\mathrm{a})$. If $K_{0}=\max (0,-f(\hat{x}))$ then for $\lambda \in \lambda_{0}^{\prime \prime}=$ $\lambda_{0}\left(K_{0}\right)$ we have, by (5.4) and (5.10),

$$
\begin{aligned}
f^{\lambda}(x) & =\max \left(\sup _{\xi \in B^{\prime}} H(\xi, x, \lambda), \sup \left\{H(\xi, x, \lambda) ; \xi \notin B^{\prime},|\xi| \leq \rho^{\prime}\right\}\right) \\
& \leq \max \left(f(\hat{x})-0,-K_{0}\right) \leq f(\hat{x}) \quad \text { for } \quad x \in \bar{B} .
\end{aligned}
$$

The proof of (b) is now complete.

5.2. Remark. (i) Assertion of Lemma 5.1 is still valid even if $\mathbf{R}$ is replaced by a normed space which may have infinite dimensions provided that $\phi(\xi)$ is replaced by $\phi(|\xi|)$. The proof presented here does not depend on locally compactness of $\mathbf{R}$ so it applies to this case with trivial modifications.

(ii) The symmetry assumption $(\Phi 1)$ is just for convenience and it may be removed if ( $\Phi 3)$ is replaced by

$$
\begin{aligned}
& \lim _{|\xi| \rightarrow \infty} \phi(\xi, 1)=\infty, \text { and } \\
& \phi(\xi, \lambda) \text { and } \phi(-\xi, \lambda) \text { are nondecreasing in } \xi \geq 0 .
\end{aligned}
$$

(iii) So far the property ( $\Phi 5)$ is not invoked. It is essentially used to prove the following Theorem which is our main result in this section.

5.3. Theorem (Convolution with faceted functions). Assume ( $\Phi 0)-$ (\$5) for $\phi$. Assume the same hypotheses of Lemma 5.1 concerning $f$. Assume that $f$ takes local maximum at $\hat{x}$ and that $f$ is not a constant function. Let $f^{\lambda}$ be a sup-convolution of $f$ by $\phi$ defined by (5.1). Then there is small $\lambda_{1} ; 0<\lambda_{1} \leq 1$ such that for $\lambda \leq \lambda_{1}$,

(i) $f^{\lambda}$ is faceted at $\hat{x}$ in $\mathbf{R}$ with slope zero and $f^{\lambda}(\hat{x})=f(\hat{x})$;

(ii) $\hat{x}$ is an interior point of the faceted region $R\left(f^{\lambda}, \hat{x}\right)$.

We set

$$
\begin{array}{lll}
a=\sup \{\boldsymbol{x} ; f(y)=f(\hat{x}) & \text { for all } & \hat{x} \leq y \leq x\} \\
b=\sup \{\boldsymbol{x} ; f(y) \leq f(\hat{x}) & \text { for all } & \hat{x} \leq y \leq x\}(\geq a) .
\end{array}
$$

Note that $b>\hat{x}$ if $f$ takes a local maximum at $\hat{x}$ and that $a<\infty$ since $f$ is not a constant. The next Lemma is a key step to prove Theorem 5.3.

5.4. Lemma. Assume the same hypotheses of Theorem 5.3 concerning $f$ and $\phi$.

(i) Assume that $a=b(>\hat{x})$. Then, for sufficiently small $\lambda$, say $\lambda<\lambda_{2}, f^{\lambda}$ is nondecreasing on $[\hat{x}, b]$, and there is $x_{\lambda}^{\prime}, \hat{x}<x_{\lambda}^{\prime} \leq b-\sigma_{\lambda}$ such that

$$
\begin{array}{lll}
f^{\lambda}(x)=f(\hat{x}) \text { for } x, \hat{x} \leq x \leq x_{\lambda}^{\prime}, \\
f^{\lambda}(x)>f(\hat{x}) \text { for } x, x_{\lambda}^{\prime}<x \leq b
\end{array}
$$


with $\sigma_{\lambda}$ as in ( $\left.\Phi 5\right)$.

(ii) Assume that $b>a(\geq \hat{\mathbf{z}})$. Assume that $f$ is uppersemicontinuous. Then for sufficiently small $\lambda$, say $\lambda<\lambda_{3}$, there are $y_{\lambda}^{1}$ and $y_{\lambda}^{2}$ with $y_{\lambda}^{2}>y_{\lambda}^{1}>\hat{x}$ and that

$$
\begin{aligned}
& f^{\lambda}(x)=f(\hat{x}) \text { for } x, \hat{x} \leq x \leq y_{\lambda}^{1} \\
& f^{\lambda}(x)<f(\hat{x}) \text { for } x, y_{\lambda}^{1}<x<y_{\lambda}^{2} .
\end{aligned}
$$

Proof of Theorem 5.3 admitting Lemma 5.4. We may assume that $f$ is uppersemicontinuous. Lemma 5.4 gives a behavior of $f^{\lambda}$ for $\boldsymbol{x}>\hat{x}$; a behavior of $f^{\lambda}$ for $x<\hat{x}$ is obtained by Lemma 5.4 by replacing $x$ by $-x$. Since $f^{\lambda}$ is continuous, the behavior of $f^{\lambda}$ so far obtained yields Theorem 5.3. We remark that Lemma 5.4 determines the value of transition numbers. It asserts that

$$
\chi_{+}\left(f^{\lambda}, \hat{x}\right)=1 \text { if } a=b \text { and } \chi_{+}\left(f^{\lambda}, \hat{x}\right)=-1 \text { if } a<b
$$

The results in Lemma 5.4 are easy to imagine since $\phi$ is faceted. However, the proof is not trivial although it relies only on elementary facts. Especially, the proof of part (ii) is complicated since we are forced to handle the case when $f$ oscillates so that it takes the value $f(\hat{x})$ infinitely many times on the interval $[a, b]$.

Proof of Lemma 5.4. Since $f$ takes its local maximum at $\hat{x}$, there is $\delta>0$ such that

$$
f(\xi) \leq f(\hat{x}) \text { for } \xi,|\xi-\hat{x}| \leq \delta .
$$

Let $x_{0}$ be a point such that $\hat{z}<x_{0}<b$. We apply Lemma 5.1 (iii) with

$$
B=\left(\hat{x}, x_{0}\right), B^{\prime}=(\hat{x}-\delta, b), K_{0}=\max (0,-f(\hat{x}))
$$

to get

$$
\sup _{\xi \notin B^{\prime}} H(\xi, x, \lambda)<-K_{0} \leq f(\hat{x}) \quad \text { for } \quad x, \hat{x} \leq x \leq x_{0}
$$

with $H(\xi, x, \lambda)=f(\xi)-\phi(\xi-x, \lambda)$, and

$$
f^{\lambda}(x) \leq f(\hat{x}) \text { for } x, \hat{x} \leq x \leq x_{0}
$$

if $\lambda<\lambda_{0}^{\prime \prime} \equiv \lambda_{0}\left(K_{0}\right)$ which also depends on $x_{0}$. We also apply Lemma 5.1(ii) with

$$
B=(\hat{x}, b), B^{\prime}=(\hat{z}-\delta, b+\delta), K_{0}=\max \left(0,-\inf _{\bar{B}} f^{*}\right)
$$


to get

$$
f^{\lambda}(x)=\sup \{H(\xi, x, \lambda) ; \hat{x}-\delta \leq \xi \leq b+\delta\} \text { for } x, \hat{x} \leq x \leq b
$$

provided that $\lambda<\lambda_{0}^{\prime} \equiv \lambda_{0}\left(K_{0}\right)$ which is well-defined if $K_{0}<\infty$.

(i) Since $a=b$, we see $f(x)=f(\hat{x})$ for $x, \hat{x} \leq x<b$. This implies

$$
\inf \left\{f^{*}(x) ; \hat{x} \leq x \leq b\right\}=f(\hat{x})>-\infty .
$$

If $\lambda<\lambda_{0}^{\prime}$ then (5.13) is valid. Since $f(\xi) \leq f(\hat{x})$ for $\xi, \hat{x}-\delta \leq \xi \leq b$ and $\phi \geq 0$ with $\phi(0)=0$, we see

$$
\begin{aligned}
H(\xi, x, \lambda) & =f(\xi)-\phi(\xi-x, \lambda) \leq f(x)-\phi(x-x, \lambda) \\
& \leq f(x)-0 \quad \text { for } \xi, \hat{x}-\delta \leq \xi \leq x
\end{aligned}
$$

for $x, \hat{x} \leq x<b$. Applying this to (5.13) we get

$$
f^{\lambda}(x)=\sup \{H(\xi, x, \lambda) ; x \leq \xi \leq b+\delta\} \text { for } x, \hat{x} \leq x \leq b
$$

for $\lambda \leq \lambda_{0}^{\prime}$ since $f^{\lambda}(x) \geq f(x) \geq H(\xi, x, \lambda)$ for $\xi, \hat{x}-\delta \leq \xi \leq x$. Since $\phi(\xi, \lambda)$ is nondecreasing in $\xi \geq 0$, one observes that

$$
H(\xi, x, \lambda)=f(\xi)-\phi(\xi-x, \lambda) \leq f(\xi)-\phi(\xi-y, \lambda)
$$

for $\hat{x} \leq \boldsymbol{x} \leq y \leq b$ and $y \leq \xi$. Since

$$
\sup \{H(\xi, x, \lambda) ; x \leq \xi \leq y\} \leq f(\hat{x})=f(y) \leq f^{\lambda}(y)
$$

(5.13') now yields $f^{\lambda}(x) \leq f^{\lambda}(y)$ for $\lambda<\lambda_{0}^{\prime}$ and $\hat{x} \leq x \leq y \leq b$. We have thus proved the monotonicity: $f^{\lambda}(x) \leq f^{\lambda}(y), \hat{x} \leq x \leq y \leq b$ for $\lambda \leq \lambda_{0}^{\prime}$.

Let $x_{0}$ be fixed with $\hat{x}<x_{0}<b$, and set $\lambda_{2}=\min \left(\lambda_{0}^{\prime}, \lambda_{0}^{\prime \prime}\right)$ so that $\lambda \leq \lambda_{2}$ implies (5.12). Since $f \leq f^{\lambda}$ by Lemma 5.1 (ii) and $a=b$ the estimate (5.12) yields

$$
f^{\lambda}(x)=f(\hat{x}) \text { for } \hat{x} \leq x \leq x_{0}, \lambda \leq \lambda_{2}
$$

By definition of $b$ there is a sequence $\xi_{j} \geq b, \xi_{j} \downarrow b$ such that $f\left(\xi_{j}\right)>f(\hat{x})$. By definition of $f^{\lambda}$ we see

$$
f^{\lambda}(x) \geq f\left(\xi_{j}\right)-\phi\left(\xi_{j}-x, \lambda\right)
$$

Assume that $b \geq x>b-\sigma_{\lambda}$. Then for sufficiently large $j$ the sequence $\xi_{j}(\downarrow b)$ satisfies $\xi_{j}-x<\sigma_{\lambda}$. We now invoke ( $\left.\Phi 5\right)$ to get

$$
\phi\left(\xi_{j}-x, \lambda\right)=0 \text { for sufficiently large } j .
$$


This is the only part ( $\Phi 5)$ is invoked in (i). We fix such $j$ and observe that

$$
f^{\lambda}(x) \geq f\left(\xi_{j}\right)-\phi\left(\xi_{j}-x, \lambda\right)=f\left(\xi_{j}\right)>f(\hat{x}) .
$$

In other words

$$
f^{\lambda}(x)>f(\hat{x}) \text { for } x, b-\sigma_{\lambda}<x \leq b \text {. }
$$

Since $f^{\lambda}$ is continuous by Lemma 5.1 (i) and $f^{\lambda}$ is nondecreasing in $[\hat{\boldsymbol{x}}, \boldsymbol{b}]$, the existence of $x_{\lambda}^{\prime}$ in (i) follows from (5.14) and (5.15). The proof of (i) is now complete.

(ii) We take $x_{0}$ with $a<x_{0}<b$ and fix $\lambda_{0}^{\prime \prime}$ in (5.11) and (5.12). Since $f$ is uppersemicontinuous, the set

$$
U=\left(a, x_{0}\right) \backslash\{x ; f(x)=f(\hat{x})\}
$$

is an open set i.e., $U$ is a disjoint union of countably many open intervals $\left\{O_{j}\right\}_{j=1}^{\infty}$. We set

$$
W_{\lambda}=\left\{j ;\left|O_{j}\right|>2 \sigma_{\lambda}\right\},
$$

where $\left|O_{j}\right|$ is the length of $O_{j}$. This set is at most finite. Let $\lambda_{4}$ be taken so that

$$
2 \sigma_{\lambda_{4}}<\max _{j \geq 1}\left|O_{j}\right|
$$

which implies that $W_{\lambda}$ is nonempty for $\lambda \leq \lambda_{4}$. We set

$$
x_{\lambda}^{1}=\inf \left\{x \in O_{j} ; j \in W_{\lambda}\right\}
$$

Since $W_{\lambda}$ is finite there is a unique $j_{0} \in W_{\lambda}$ with $x_{\lambda}^{1}=\inf O_{j_{0}}$. We then set

$$
x_{\lambda}^{2}=\sup O_{j_{0}}\left(\leq x_{0}\right) \text {. }
$$

We shall prove that if $\lambda \leq \lambda_{3}$ with $\lambda_{3}=\min \left(\lambda_{4}, \lambda_{0}^{\prime \prime}\right)$ then

$$
\begin{gathered}
f^{\lambda}(x)=f(\hat{x}) \text { for } x, \hat{x} \leq x \leq x_{\lambda}^{1}+\sigma_{\lambda} \\
f^{\lambda}(x)<f(\hat{x}) \text { for } x, x_{\lambda}^{1}+\sigma_{\lambda}<x<x_{\lambda}^{2}-\sigma_{\lambda} .
\end{gathered}
$$

Note that $x_{\lambda}^{1}+\sigma_{\lambda}<x_{\lambda}^{2}-\sigma_{\lambda}$ since $\left|O_{j_{0}}\right|>2 \sigma_{\lambda}$.

By definition of $W_{\lambda}$ for $x, \hat{x} \leq x \leq x_{\lambda}^{1}+\sigma_{\lambda}$ there is $\xi_{0}, x-\sigma_{\lambda}<\xi_{0}<x+\sigma_{\lambda}$ such that

$$
f\left(\xi_{0}\right)=f(\hat{x})
$$


which yields

$$
\begin{aligned}
f^{\lambda}(x) & \geq f\left(\xi_{0}\right)-\phi\left(\xi_{0}-x, \lambda\right) \\
& =f(\hat{x})-0
\end{aligned}
$$

The property (5.16) now follows from (5.12).

It remains to prove (5.17). Assume that $x$ fulfills $x_{\lambda}^{1}+\sigma_{\lambda}<x<x_{\lambda}^{2}-\sigma_{\lambda}$ and $\lambda \leq \lambda_{3}$. By definition of $O_{j 0}$ it follows that

$$
H(\xi, x, \lambda)=f(\xi)-\phi(\xi-x, \lambda)<f(\hat{x})-0 \text { for } \xi, x_{1}^{\lambda}<\xi<x_{2}^{\lambda} .
$$

Since $\lambda \leq \lambda_{3} \leq \lambda_{0}^{\prime \prime},(5.11)$ yields

$$
\sup \{H(\xi, x, \lambda), \xi \leq \hat{x}-\delta \text { or } \xi \geq b\}<f(\hat{x}) .
$$

By definition of $\delta$ and $b$ if $\xi \in[\hat{x}-\delta, b]$ then $f(\xi) \leq f(\hat{x})$ so that

$$
H(\xi, x, \lambda) \leq f(\hat{x})-\phi(\xi-x, \lambda) .
$$

If $\xi$ is outside $\left(x_{\lambda}^{1}, x_{\lambda}^{2}\right)$, then $\phi(\xi-x, \lambda)>0$ so that

$$
H(\xi, x, \lambda)<f(\hat{x}) \text { for } \xi \in[\hat{z}-\delta, b] \backslash\left(x_{\lambda}^{\prime}, x_{\lambda}^{2}\right) \text {. }
$$

Since $f$ is uppersemicontinuous, (5.18) and (5.20) implies

$$
\sup \{H(\xi, x, \lambda) ; \hat{x}-\delta \leq \xi \leq b\}<f(\hat{x}) .
$$

This together with (5.19) yields

$$
f^{\lambda}(x)=\sup _{\xi \in \mathbf{R}} H(\xi, x, \lambda)<f(\hat{x})
$$

for $x, x_{\lambda}^{1}+\sigma_{\lambda}<x<x_{\lambda}^{2}-\sigma_{\lambda}$.

In $\S 7$ we use a sup-convolution of a function by $\vartheta$ defined in (5.2). There is an advantage to use this special $\vartheta$ since it fulfills a composition rule

$$
f^{\beta}(x)=\vartheta(x, \lambda-\beta) \text { for } 0<\beta<\lambda \text { with } f(x)=\vartheta(x, \lambda) .
$$

We conclude this section to prove a more general composition rule which includes (5.21) as a special case. For $\rho \geq 0$ and $\lambda>0$ let $\vartheta(x, \rho, \lambda)$ be of the form

$$
\vartheta(x, \rho, \lambda)= \begin{cases}(x-\rho)^{2} / \lambda ; & x>\rho \\ 0 ; & |x| \leq \rho \\ (x+\rho)^{2} / \lambda ; & x<-\rho\end{cases}
$$


5.5. Lemma(Composition). (i) For $0 \leq \alpha \leq \rho, 0<\beta<\lambda$,

$$
\vartheta(x, \rho-\alpha, \lambda-\beta)=\sup _{\xi \in \mathbb{R}}\{\vartheta(\xi, \rho, \lambda)-\vartheta(\xi-x, \alpha, \beta)\} \quad \text { for } \quad x \in \mathbf{R} .
$$

(ii) For $0 \leq \alpha_{i}, 0<\beta_{i}(i=1,2)$ with $\alpha_{1}+\alpha_{2} \leq \rho, \beta_{1}+\beta_{2}<\lambda$ we have

$$
\begin{aligned}
& \vartheta\left(x-y, \rho-\left(\alpha_{1}+\alpha_{2}\right), \lambda-\left(\beta_{1}+\beta_{2}\right)\right) \\
&= \sup _{\xi} \sup _{\eta}\left\{(\xi-\eta, \rho, \lambda)-\vartheta\left(\xi-x, \alpha_{1}, \beta_{1}\right)-\vartheta\left(\eta-y, \alpha_{2}, \beta_{2}\right)\right\} \\
& \text { for } x, y \in \mathbf{R} .
\end{aligned}
$$

Proof. (i) By an elementary calculus one can evaluate the maximum of the right hand side to get the desired identity. We give a noncomputational proof for completeness.

We set

$$
H(\xi, x)=\vartheta(\xi, \rho, \lambda)-\vartheta(\xi-x, \alpha, \beta), \quad f(x)=\sup _{\xi \in \mathbb{R}} H(\xi, x)
$$

by suppressing parameters.

(a) If $|x| \leq \rho-\alpha$, then $f(x)=0$. Indeed, from $\lambda>\beta, \rho \geq \alpha$ it follows that

$$
\vartheta(\xi-x, \alpha, \beta) \geq \vartheta(\xi-x, \alpha, \lambda) \geq \vartheta(\xi, \rho, \lambda)
$$

which yields $H(\xi, x) \leq 0$ for all $\xi \in \mathbf{R}$. Since $H(\xi, x) \geq H(x, x) \geq 0$, we now obtain $f(x)=0=\vartheta(x, \rho-\alpha, \lambda-\beta)$ for $|x| \leq \rho-\alpha$.

(b) If $x>\rho-\alpha$, then

$$
f(x)=\sup \{H(\xi, x) ; \xi \geq x+\alpha\} .
$$

Indeed, for $\xi,|\xi| \leq x+\alpha$, we see

$H(\xi, x)=\vartheta(\xi, \rho, \lambda)-0 \leq \vartheta(x+\alpha, \rho, \lambda)-\vartheta(x+\alpha-x, \alpha, \beta)=H(x+\alpha, x)$.

For $\xi \leq-(x+\alpha)$ we see

$$
\begin{aligned}
H(\xi, \boldsymbol{x}) & =\vartheta(\xi, \rho, \lambda)-\vartheta(\boldsymbol{x}-\xi, \alpha, \beta) \\
& \leq \vartheta(-\xi, \rho, \lambda)-\vartheta(-\xi-\boldsymbol{x}, \alpha, \beta)=H(-\xi, \boldsymbol{x})
\end{aligned}
$$

since $x-\xi \geq-\xi-x \geq \alpha$. We thus obtain (5.22). An elementary observation shows

$$
\sup _{\xi \in \mathbb{R}}\left\{\frac{(\xi-\rho)^{2}}{\lambda}-\frac{(\xi-x-\alpha)^{2}}{\beta}\right\}=\sup _{\xi \geq x+\alpha}\left\{\frac{(\xi-\rho)^{2}}{\lambda}-\frac{(\xi-x-\alpha)^{2}}{\beta}\right\}
$$


since $\boldsymbol{x}+\alpha>\rho, \lambda>\beta$. Since

$$
H(\xi, x)=\frac{(\xi-\rho)^{2}}{\lambda}-\frac{(\xi-x-\alpha)^{2}}{\beta} \text { for } \xi \geq x+\alpha,
$$

from (5.22) it now follows that

$$
\begin{aligned}
f(x) & =\sup _{\xi \in \mathbf{R}}\left\{\frac{(\xi-\rho)^{2}}{\lambda}-\frac{(\xi-x-\alpha)^{2}}{\beta}\right\} \\
& =\sup _{\eta \in \mathbf{R}}\left\{\frac{\eta^{2}}{\lambda}-\frac{(\eta-z)^{2}}{\beta}\right\}, \quad z=x-(\rho-\alpha) .
\end{aligned}
$$

Since $\beta<\lambda$, the last supremum is attained only at $\eta_{0}=\lambda z /(\lambda-\beta)$ we see

$$
f(x)=\frac{\eta_{0}^{2}}{\lambda}-\frac{\left(\eta_{0}-z\right)^{2}}{\beta}=\frac{z^{2}}{\lambda-\beta} .
$$

This shows $f(x)=\vartheta(x, \rho-\alpha, \lambda-\beta)$ for $x>\rho-\alpha$. The proof for $x \leq-(\rho-\alpha)$ is the same so is omitted.

(ii) Using (i) twice we obtain the desired identity.

\section{§6. Equivalent definitions of solutions.}

To show comparison theorem (and existence theorem) it is convenient to introduce other versions of definitions of solutions. In our original definition our test function $\psi$ is admissible on whole $Q=(0, T) \times \Omega$. However, it turns out that we only need some admissibility of $\psi$ near the point $(\hat{t}, \hat{x}) \in Q$ such that

$$
\max _{Q}\left(u^{*}-\psi\right)=(u-\psi)(\hat{t}, \hat{z}) \text {. }
$$

To be precise we introduce several notions of admissible functions. In this section $\Omega$ is assumed to be a (possibly unbounded) open interval.

6.1. Definition (Locally admissible functions). Let $(\hat{t}, \hat{\boldsymbol{x}})$ be a point in $Q$. A function $\psi \in C(Q)$ is locally admissible near $(\hat{t}, \hat{x})$ in $Q$ if there is a rectangular neighborhood $\hat{Q} \subset Q$ of $(\hat{t}, \hat{x})$ such that the restriction $\left.\psi\right|_{\hat{Q}}$ of $\psi$ on $\hat{Q}$ belongs to $A_{p}(\hat{Q})$. Since $\hat{Q}$ is rectangular, it is of form

$$
\hat{Q}=J \times \hat{\Omega}
$$

with open intervals $J$ and $\hat{\Omega}$.

By definition if $\hat{p}=\psi_{x}(\hat{t}, \hat{x}) \in P$ then $\psi(\hat{t}, \cdot)$ is faceted at $\hat{x}$ in $\hat{\Omega}$ with slope $\hat{p}$. 
Our assumption $\left.\psi\right|_{\hat{Q}} \in A_{P}(\hat{Q})$ implies that $\left.\psi(\hat{t}, \cdot)\right|_{\hat{\Omega}} \in C_{P}^{2}(\hat{\Omega})$. In particular, the faceted region $R(\psi(\hat{t}, \cdot), \hat{x})$ should be contained in $\hat{\boldsymbol{\Omega}}$.

6.2. Semijets. We recall definition of parabolic semi jets in [CIL]. Let $\varphi$ be a function on $Q$ and $(\hat{t}, \hat{x}) \in Q$. The set of parabolic semijets is of the form

$$
\begin{aligned}
& \mathcal{P}_{Q}^{2,+} \varphi(\hat{t}, \hat{x})=\{(\tau, p, X) \in \mathbf{R} \times \mathbf{R} \times \mathbf{R} ; \\
& \varphi(t, x)-\varphi(\hat{t}, \hat{x}) \leq \tau(t-\hat{t})+p(x-\hat{x})+\frac{1}{2} X(x-\hat{x})^{2} \\
& \left.+o\left(|t-\hat{t}|+|x-\hat{x}|^{2}\right) \text { as }(t, x) \rightarrow(\hat{t}, \hat{x})\right\} .
\end{aligned}
$$

The set $\mathcal{P}_{Q}^{2,-} \varphi(\hat{t}, \hat{x})$ is defined by

$$
\mathcal{P}_{Q}^{2,-} \varphi(\hat{t}, \hat{x})=-\left(\mathcal{P}_{Q}^{2,+}(-\varphi)\right)(\hat{t}, \hat{x}) .
$$

We often write $\mathcal{P}^{ \pm}$instead of $\mathcal{P}_{Q}^{2, \pm}$.

If $\varphi(\hat{t}, \cdot)$ is faceted at $\hat{x}, \mathcal{P}^{+}$is not enough to discuss behavior of $\varphi$ near the faceted region $R(\varphi(\hat{t}, \cdot), \hat{x})$. We are interested in defining upper time derivative on the faceted region. For this purpose we introduce semineighborhoods of faceted region depending on the local behavior of $\varphi(\hat{t}, \cdot)$.

6.3. Semineighborhoods. Let $f \in C(\Omega)$ be faceted at $x_{0}$ with slope $p_{0}$. Let $\delta_{+}$and $\delta_{-}$be (small) positive numbers. Let $R\left(f, x_{0}\right)$ denote the faceted region of $f$ containing $x_{0}$. We set

$$
\begin{aligned}
& N_{+}\left(f, x_{0} ; \delta_{+}\right)=\left\{x \in \Omega ; \sup R\left(f, x_{0}\right)<x \leq \sup R\left(f, x_{0}\right)+\delta_{+}\right\}, \\
& N_{-}\left(f, x_{0} ; \delta_{-}\right)=\left\{x \in \Omega ; \inf R\left(f, x_{0}\right)-\delta_{-} \leq x<\inf R\left(f, x_{0}\right)\right\} .
\end{aligned}
$$

Our semineighborhood depends on $\chi_{ \pm}\left(f, x_{0}\right)$.

(i) If $\chi_{+}\left(f, x_{0}\right)=\chi_{-}\left(f, x_{0}\right)=-1$ we set:

$$
\tilde{N}^{-1}\left(f, x_{0} ; \delta_{+}, \delta_{-}\right)=R\left(f, x_{0}\right) \cup N_{+}\left(f, x_{0} ; \delta_{+}\right) \cup N_{-}\left(f ; x_{0} ; \delta_{-}\right) .
$$

(ii) If $\chi+\left(f, x_{0}\right)=1$ and $\chi-\left(f, x_{0}\right)=-1$ we set

$$
\tilde{N}^{-1}\left(f, x_{0} ; \delta_{+}, \delta_{-}\right)=R\left(f, x_{0}\right) \cup N_{-}\left(f, x_{0} ; \delta_{-}\right) .
$$

(iii) If $\chi_{+}\left(f, x_{0}\right)=-1$ and $\chi_{-}\left(f, x_{0}\right)=1$ we set

$$
\tilde{N}^{-1}\left(x_{0} ; \delta_{+}, \delta_{-}\right)=R\left(f, x_{0}\right) \cup N_{+}\left(f, x_{0} ; \delta_{+}\right)
$$

(iv) If $\chi_{+}\left(f, x_{0}\right)=\chi_{-}\left(f, x_{0}\right)=1$, we set

$$
\tilde{N}^{-1}\left(f, x_{0} ; \delta_{+}, \delta_{-}\right)=R\left(f, x_{0}\right) \text {. }
$$


The set $\tilde{N}^{+1}\left(f, x_{0} ; \delta_{+}, \delta_{-}\right)$is defined by

$$
\tilde{N}^{+1}\left(f, x_{0} ; \delta_{+}, \delta_{-}\right)=\tilde{N}^{-1}\left(-f, x_{0} ; \delta_{+}, \delta_{-}\right) \text {. }
$$

In other words, $\tilde{N}^{+1}$ is defined in the same way by interchanging 1 and -1 in (i)-(iv). We often suppress $\delta_{+}$and $\delta_{-}$of $\tilde{N}^{ \pm 1}$ and simply write by $\tilde{N}^{ \pm}\left(f, x_{0}\right)$.

6.4. Upper time derivatives. A function $\omega:[0, \infty) \rightarrow[0, \infty)$ is called modulus if $\omega$ is a nondecreasing continuous function with $\omega(0)=0$. For a function $\varphi$ on $Q$ we define

$$
\begin{aligned}
& \mathcal{T}_{P}^{+} \varphi(\hat{t}, \hat{z})=\{\tau \in R ; \quad \text { there are a modulus } \omega \text { and three positive numbers } \\
& \qquad, \delta, \delta_{+}, \delta_{-} \text {such that }
\end{aligned}
$$

$$
\begin{aligned}
& \varphi(t, x)-\varphi(\hat{t}, \hat{x}) \leq \tau(t-\hat{t})+p(x-\hat{x})+\omega(|t-\hat{t}|)|t-\hat{t}| \\
& \text { for } \left.(t, x) \in(\hat{t}-\delta, \hat{t}+\delta) \times \hat{N}^{-1}\left(\varphi(\hat{t}, \cdot), \hat{x}, \delta_{+}, \delta_{-}\right)\right\}
\end{aligned}
$$

provided that $\varphi(\hat{t}, \cdot)$ is $P$-faceted at $\hat{x} \in \Omega$ with slope $p$ in $\Omega$. If $\varphi(\hat{t}, \cdot)$ is not $P$-faceted at $\hat{x}$, we set $\mathcal{T}_{P}^{+} \varphi(\hat{t}, \hat{x})=\emptyset$. The set $\mathcal{T}_{P}^{-} \varphi(\hat{t}, \hat{x})$ is defined by

$$
\mathcal{T}_{P}^{-} \varphi(\hat{t}, \hat{\boldsymbol{x}})=-\left(\mathcal{T}_{(-P)}^{+}(-\varphi)\right)(\hat{t}, \hat{x})
$$

The set $\mathcal{T}_{\boldsymbol{p}}^{-}$is roughly the set of upper time derivatives which are uniform near the faceted region since error term $\omega(|t-\hat{t}|)|t-\hat{t}|$ is independent of $x$ at least on the faceted region.

6.5. Definition (Admissible super functions). Let $\varphi: Q=(0, T) \times \Omega \rightarrow$ $\mathbf{R}$ be an upper semicontinuous function. Let $(\hat{t}, \hat{x})$ be a point in $Q$. Assume that $\varphi(t, \cdot) \in C(\Omega)$ for $t$ near $\hat{t}$. We say $\varphi$ is an (infinitesimally) admissible super function at $(\hat{t}, \hat{x})$ in $Q$ if one of following conditions holds.

(A) The function $\varphi(\hat{t}, \cdot)$ is $P$-faceted at $\hat{x}$ in $\Omega$ and $\hat{x} \in \operatorname{int} R(\varphi(\hat{t}, \cdot), \hat{z})$, i. e., $\hat{x}$ is an interior point of the faceted region of $\varphi(\hat{t}, \cdot)$ containing $\hat{x}$. The set $\mathcal{T}_{P}^{+} \varphi(\hat{t}, \hat{x})$ is nonempty.

(B) There is $(\tau, p, X) \in \mathcal{P}^{+} \varphi(\hat{t}, \hat{x})$ with $p \notin P$.

(C) The function $\varphi(\hat{t}, \cdot)$ is $P$-faceted at $\hat{x}$ in $\Omega$ but $\hat{x} \in \delta R(\varphi(\hat{t}, \cdot), \hat{x})$. The function $\varphi$ is locally admissible near $(\hat{t}, \hat{x})$ in $Q$.

We say $\varphi$ is an admissible sub function at $(\hat{t}, \hat{x})$ in $Q$ if $-\varphi$ is an admissible super function with $P$ replaced by $-P$. If $\varphi$ is locally admissible near $(\hat{t}, \hat{x})$ in $Q$, it is easy to check (A), (B) or (C) so that $\varphi$ is an admissible super function at $(\hat{t}, \hat{x})$ in $Q$. 
6.6. Definition (Subsolution -infinitesimal version). A real valued function $u$ on $Q$ is a subsolution in infinitesimal sense of (E) if $u^{*}<\infty$ in $[0, T) \times \bar{\Omega}$ and the following conditions are fulfilled. For each $(\hat{t}, \hat{x})$ let $\varphi$ be an admissible super function at $(\hat{t}, \hat{x})$ in $Q$ such that (2.2) holds. Then

(i) $\tau+F\left(\hat{t}, \varphi_{s}(\hat{t}, \hat{x}), \Lambda_{W}(\varphi(\hat{t}, \cdot), \hat{x})\right) \leq 0$

for all $\tau \in \mathcal{T}_{P}^{+} \varphi(\hat{t}, \hat{x})$ if $(A)$ in $\$ 6.5$ holds;

(ii) $\tau+F\left(\hat{t}, p, W^{\prime \prime}(p) X\right) \leq 0$

for all $(\tau, p, X) \in \mathcal{P}^{+} \varphi(\hat{t}, \hat{x})$ if (B) in $\$ 6.5$ holds and

(iii) (2.2) is valid with $\psi=\varphi$ if (C) in $\$ 6.5$ holds.

The definition of supersolution is given by replacing $u^{*}(<\infty)$ by $u_{*}(>$ $-\infty)$, max by min, super function by sub function, $\mathcal{T}_{P}^{+}$by $\mathcal{T}_{P}^{-}, \mathcal{P}^{+}$by $\mathcal{P}^{-}$ and the inequalities in (i)(ii) and (2.2) by the opposite one. We note that if $F$ is continuous and $W^{\prime \prime}$ is continuous near $p$, then $\mathcal{P}^{+}$may be replaced by $\overline{\mathcal{P}}_{Q}^{2,+}$ the closure of $\mathcal{P}^{+}=\mathcal{P}_{Q}^{2,+}$ in the sense of semijets [CIL]:

$$
\begin{gathered}
\overline{\mathcal{P}}_{Q}^{2,+} \varphi(\hat{t}, \hat{\boldsymbol{x}})=\left\{(\tau, p, X) ; \text { there are sequences } x_{n} \rightarrow \hat{\boldsymbol{x}}, t_{n} \rightarrow \hat{t}\right. \\
\tau_{n} \rightarrow \tau, X_{n} \rightarrow X \text { satisfying } \varphi\left(t_{n}, x_{n}\right) \rightarrow \varphi(t, x) \\
\left.\left(\tau_{n}, p_{n}, X_{n}\right) \in \mathcal{P}_{Q}^{2,+} \varphi\left(t_{n}, x_{n}\right),\left(t_{n}, x_{n}\right) \in Q\right\}
\end{gathered}
$$

6.7. Definition (Subsolution -local version). A real valued function $u$ on $Q$ is a subsolution in local sense of (E) if $u^{*}<\infty$ in $[0, T) \times \bar{\Omega}$ and (2.1) holds for all $(\hat{t}, \hat{x}) \in Q$ and for all $\psi(\in C(Q))$ which is locally admissible near $(\hat{t}, \hat{x})$ in $Q$ and fulfills (2.2). The definition of supersolution is given by replacing $u^{*}$ by $u_{*}, \max$ and $\min$ and the inequality (2.1) by the opposite one as before.

Our main goal in this section is to show that Definitions 6.6, 6.7 are equivalent to Definition 2.6.

6.8. Theorem (Local version v.s. original global version). A real valued function on $Q$ is a subsolution (resp. supersolution) of (E) if and only if it is a subsolution (resp. supersolution) in local sense of $(E)$.

6.9. Theorem (Local version v.s. infinitesimal version). Assume that (F1) holds. A real valued function on $Q$ is a subsolution (resp. supersolution) in local sense of (E) if and only if it is a subsolution (resp. supersolution) in infinitesimal sense of $(E)$.

6.10. Remark. At the first glance our definition of admissible super function is rather strange. In the definition of $\mathcal{T}_{P}^{+}$we are tempting to replace $\tilde{N}^{-1}$ by $R(\varphi(\hat{t}, \cdot), \hat{\boldsymbol{x}})$. However, if we do so, then subsolution might not be a subsolution in infinitesimal sense. We are also tempting to replace $\tilde{N}^{-1}$ by $\tilde{N}^{+1} \cup \tilde{N}^{-1}$, a neighborhood of $R(\varphi(\hat{t}, \cdot), \hat{\boldsymbol{x}})$. This modification is good 
to prove Theorem 6.9. However, test functions constructed in the proof of Comparison Theorem (\$7) might not be admissible under this modification. Note that $\$ 7$ is the only place the infinitesimal version of definition is invoked. In $\$ 7$ there is no situation that $\hat{z}$ is a boundary point of $R(\varphi(\hat{t}, \cdot), \hat{z})$ so the definition of local version at such $\hat{x}$ is inherited in Definitions 6.5 and 6.6.

\section{A. Preliminary Lemmas.}

To prove Theorems 6.8 and 6.9 we prepare several lemmas.

6.11. Extension Lemma. Let $I$ be an open interval in $(0, T)$ and $J$ be an bounded open interval in $\Omega$. Assume that $\varphi \in C(Q)(Q=(0, T) \times \Omega)$ fulfills

$$
\left.\varphi\right|_{I \times J} \in A_{P}(I \times J) .
$$

Let $I_{1}$ and $J_{1}$ be open intervals such that $\bar{I}_{1} \subset I$ and $\bar{J}_{1} \subset J$. Then there is a function $\psi \in A_{P}(Q)$ such that

$$
\varphi \leq \psi \text { in } Q \text { and } \varphi=\psi \text { in } \bar{I}_{1} \times \bar{J}_{1} \text {. }
$$

Proof. Step 1. We shall prove: $I_{k}$ and $J_{k}$ (with $k=2,3$ ) be open intervals such that

$$
\begin{aligned}
& \bar{I}_{h} \subset I_{h+1} \quad \text { and } \quad \bar{I}_{3} \subset I \quad(k=1,2), \\
& \bar{J}_{k} \subset J_{h+1} \quad \text { and } \bar{J}_{3} \subset J \quad(k=1,2) .
\end{aligned}
$$

Then there are nonnegative functions $f_{2} \in C(\Omega)$ and $g_{2} \in C(0, T)$ such that

$$
\begin{aligned}
& f_{2} \equiv 0 \text { in } J_{2}, \quad g_{2} \equiv 0 \text { in } I_{2}, \\
& \varphi(t, x) \leq f_{2}(x)+g_{2}(t), \quad(t, x) \in Z=Q \backslash\left(I_{3} \times J_{3}\right) .
\end{aligned}
$$

Indeed, we take $d \in C^{1}(\Omega)$ that satisfies

(a) $d \equiv 0$ in $J_{3}$

(b) $d^{\prime}>0$ in the right of $J_{3} ; d^{\prime}<0$ in the left of $J_{3}$

(c) $d(x) \rightarrow+\infty$ as $x$ tends to the boundary of $\Omega$.

We set

$$
J(r)=\{x \in \Omega ; d(x)<r\}
$$

to see $J(0)=J_{3}$ and $\cup_{r} \geq 0 J(r)=\Omega$. Similarly, we take $\bar{d} \in C^{1}(0, T)$ that satisfies (a), (b), (c) with $J_{3}$ and $\Omega$ replaced by $I_{3}$ and $(0, T)$, respectively. We also set

$$
I(r)=\{t \in(0, T) ; \bar{d}(x)<r\}
$$

and

$$
h(r)=\max \{\max (\varphi(t, x), 0),(t, x) \in Z,(t, x) \in \overline{I(r)} \times \overline{J(r)}\}
$$


Since $\overline{I(r)} \times \overline{J(r)}$ is compact and $\varphi$ is continuous $h$ is continuous in $[0, \infty)$. There are nonnegative function $f_{2} \in C(\Omega)$ and $g_{2} \in C(0, T)$ such that

$$
\begin{aligned}
f_{2}(x) & =h(d(x)) & & \text { for } x \in \Omega \backslash J_{3}, \\
& =0 & & \text { for } x \in J_{2}, \\
g_{2}(t) & =h(\bar{d}(t)) & & \text { for } t \in(0, T) \backslash I_{3}, \\
& =0 & & \text { for } t \in I_{2} .
\end{aligned}
$$

By definition

$$
\begin{aligned}
\varphi(t, x) & \leq h(\max (d(x), \bar{d}(t))) \\
& \leq \max \left(f_{2}(x), g_{2}(t)\right) \\
& \leq f_{2}(x)+g_{2}(t) \text { for }(t, x) \in Z
\end{aligned}
$$

since both $f_{2}$ and $g_{2}$ are nonnegative. This completes the proof of Step 1 .

Step 2. Since $\left.\varphi\right|_{I \times J} \in A_{P}(I \times J)$, there is $f_{1} \in C_{P}^{2}(J)$ and $g_{1} \in C^{1}(I)$ such that

$$
\varphi(t, x)=f_{1}(x)+g_{1}(t) \text { in } I \times J .
$$

Let $\theta \in C(\Omega)$ and $\rho \in C(0, T)$ satisfy

$$
\begin{aligned}
& 0 \leq \theta \leq 1, \theta \equiv 1 \text { in } J_{3}, \theta \equiv 0 \text { in a neighborhood of } \Omega \backslash J, \\
& 0 \leq \rho \leq 1, \rho \equiv 1 \text { in } I_{3}, \rho \equiv 0 \text { in a neighborhood of }(0, T) \backslash I .
\end{aligned}
$$

Then, it is easy to see that $f_{3}=\theta f_{1}+f_{2} \in C(\Omega)$ and $g_{3}=\rho g_{1}+g_{2} \in C(0, T)$ fulfills

$$
\begin{aligned}
& f_{3}=f_{1} \text { in } J_{2}, g_{3}=g_{1} \text { in } I_{2}, \\
& \varphi(t, x) \leq f_{3}(x)+g_{3}(t),(t, x) \in Q,
\end{aligned}
$$

where $f_{1}$ and $g_{1}$ are extended by zero outside $J$ and $I$ respectively.

Step 3. To complete the proof it suffices to find $g \in C^{1}(0, T)$ and $f \in C_{P}^{2}(\Omega)$ such that $\underline{g} \geq g_{3}$ on $(0, T)$ with $g=g_{3}$ in $\bar{I}_{1}$ and that $f \geq f_{3}$ on $\Omega$ with $f=f_{3}$ in $\bar{J}_{1}$. Since $g_{3} \in C(0, T)$ fulfills $g_{3}=g_{1}$ in $I_{2}$, so that $g_{3}$ is $C^{1}$ in a neighborhood of $\bar{I}_{1}$, it is easy to find such a function $g$. If $J_{2} \backslash J_{1}$ is contained in one faceted region of $f_{1}$ we take a nonnegative $C^{2}$ function $\sigma$ supported in $J_{2} \backslash \bar{J}_{1}$ such that $\left(f_{1}+\sigma\right)^{\prime}$ does not belong to $P$ at the boundary of some neighborhood $J^{\prime}$ of $J_{1}$ in $J_{2}$ and that $f_{1}+\left.\sigma\right|_{J_{2}} \in C_{P}^{2}\left(J_{2}\right)$. Replacing $f_{3}$ by $f_{3}+\sigma$ we may assume that $f_{3} \in C(\Omega)$ fulfills

$$
f_{3} \in C_{P}^{2}\left(J_{2}\right) \text { and } f_{3}^{\prime}(x) \notin P \text { for all } x \in \delta J^{\prime} \text {. }
$$

The proof is now complete, if we admit the next lemma, which yields a desired $f$. 
6.12. $C_{P}^{2}$ extension Lemma. Let $J^{\prime}, J_{2}$ and $\Omega$ be (possibly unbounded) open intervals such that $\vec{J} \subset J_{2}, \overline{J_{2}} \subset \Omega$. Assume that $f_{0} \in C(\Omega)$ satisfies

$$
\left.f_{0}\right|_{J_{2}} \in C_{P}^{2}\left(J_{2}\right) \text { and } f_{0}^{\prime}(x) \notin P \text { for all } x \in \partial J^{\prime} .
$$

Then there is a function $f \in C_{P}^{2}(\Omega)$ such that $f \geq f_{0}$ in $\Omega$ and that $f=f_{0}$ in $J^{\prime}$. If $f_{0}^{\prime \prime} \geq 0$ in $J_{2}, f$ can be taken so that $f^{\prime \prime} \geq 0$ in $\Omega$.

Proof. For sufficiently small open neighborhood $J^{\prime \prime}$ of $\bar{J}^{\prime}$ in $J_{2}$ there is $\bar{f}_{0} \in$ $C^{2}(\Omega)$ such that $\bar{f}_{0}=f_{0}$ in $\bar{J}^{\prime \prime}$ and $f_{0} \leq \bar{f}_{0}$ in $\Omega$ and that $\left.\bar{f}_{0}\right|_{J^{\prime \prime}} \in C_{P}^{2}\left(J^{\prime \prime}\right)$ since $f_{0}^{\prime}(x) \notin P$ near $\delta J^{\prime}$. So we may assume $f_{0} \in C^{2}(\Omega)$ by replacing $J_{2}$ by $J^{\prime \prime}$. We then apply the next elementary but important lemma on intervals $\Omega \backslash J^{\prime}=\left(b^{\prime}, a^{\prime}\right) \cup(a, b)$ and obtain a function $f$ on $\Omega \backslash J^{\prime}$ with $f^{(k)}\left(a^{\prime}\right)=$ $f_{0}^{(h)}\left(a^{\prime}\right), f^{(h)}(a)=f_{0}^{(h)}(a)(k=0,1,2), f \in C_{P}^{2}\left(\Omega \backslash \bar{J}^{\prime}\right)$ and $f \geq f_{0}$ on $\Omega \backslash J^{\prime}$. If we set $f=f_{0}$ on $\vec{J}^{\prime}$, so that $f \in C_{P}^{2}(\Omega)$, then $f \geq f_{0}$ in $\Omega$ and $f=f_{0}$ in $J^{\prime}$. If $f_{0}^{\prime \prime} \geq 0$ on $J_{2}$ our $f$ satisfies $f^{\prime \prime} \geq 0$ in $\Omega$ as noted in the next lemma. Thus the proof of Lemma 6.12 is complete by admitting the next lemma.

6.13. Lemma. Assume that $a<b \leq \infty$. For $f_{0} \in C^{2}[a, b)$ we assume that $f_{0}^{\prime}(a) \notin P$. Then there is $f \in C^{2}[a, b) \cap C_{P}^{2}(a, b)$ such that $f=f_{0}$ at $x=a$ up to second derivatives, i.e. $f^{(k)}(a)=f_{0}^{(k)}(a)(k=0,1,2)$ and that $f_{0} \leq f$ in $[a, b)$. If $f_{0}^{(2)}(a) \geq 0$, then $f$ can be taken so that $f^{\prime \prime} \geq 0$ on $[a, b)$. The same assertion holds if we replace $[a, b)$ by $(b, a]$ if $-\infty \leq b<a$.

Proof. Since the proof is the same for $b>a$ we may assume $a<b$. We may assume $a=0$ by a translation. We may assume $f_{0}^{\prime}(0)=0$ by adding $-f_{0}^{\prime}(0) x$ to $f$ and replacing $P$ by $P-f_{0}^{\prime}(0)$.

We may also assume that there is $x_{1} \geq 0$ such that $f_{0}^{\prime}(x) \notin P$ for $0 \leq x \leq$ $x_{1}$ and that $f_{0}^{\prime \prime} \geq 0$ on $\left[x_{1}, b\right)$ with $f_{0}^{\prime}\left(x_{1}\right)=0$. Indeed, if $f_{0}^{\prime \prime}(0) \geq 0$, we take $\sigma \in C[0, b)$ such that $\sigma \geq f_{0}^{\prime \prime}$ in $[0, b), \sigma(0)=f_{0}^{\prime \prime}(0)$ and $\sigma \geq 0$. We then set

$$
\bar{f}_{0}(x)=f_{0}(0)+\int_{0}^{x} d y \int_{0}^{y} d z \sigma(z)
$$

so that $\bar{f}_{0} \geq f_{0}, \bar{f}_{0}^{\prime \prime} \geq 0$ on $[0, b)$ with $f_{0}^{(k)}(0)=\bar{f}_{0}^{(k)}(0)(k=1,2)$. In this case $x_{1}$ is chosen to be equal to zero and $f^{\prime \prime} \geq 0$ on $[0, b)$. If $f_{0}^{\prime \prime}(0)<0$, for each $\epsilon>0$ we take $\sigma \in C[0, b)$ such that $\sigma \geq f_{0}^{\prime \prime}$ in $[0, b), \sigma(0)=f_{0}^{\prime \prime}(0)(<0), \sigma \geq 0$ in $[\epsilon, b), \sigma<0$ in $[0, \epsilon)$ and that $\sigma$ is increasing in $x$. Let $\bar{f}_{0}$ be defined as in the preceding formula of $\bar{f}_{0}$ but with this new $\sigma$. It turns out that if $\epsilon>0$ is sufficiently small, then for $\bar{p}=\inf \left\{\vec{f}_{0}^{\prime}(x) ; 0 \leq x \leq b\right\}\left(=\vec{f}_{0}^{\prime}(\epsilon)\right)$ we have $[\bar{p}, 0] \cap P=\emptyset$. Moreover, there is $x_{1}>\epsilon$ such that $\vec{f}_{0}^{\prime}\left(x_{1}\right)=0$. Since $\bar{f}_{0}^{\prime \prime}=\sigma \geq 0$ on $[\epsilon, b)$, this $\bar{f}_{0}\left(\geq f_{0}\right)$ has the required property of $f_{0}$ in this paragraph with $f_{0}^{(k)}(0)=\bar{f}_{0}^{(k)}(0)(k=1,2)$. 
It is easy to construct $h \in C^{1}\left[x_{1}, b\right)$ such that

$$
\begin{aligned}
& h \geq f_{0}^{\prime}(\geq 0), h^{\prime} \geq 0 \text { on }\left[x_{1}, b\right) \\
& h=f_{0}^{\prime} \text { near } x=x_{1} \text { with } x \geq x_{1}
\end{aligned}
$$

and that $\left\{x \in\left(x_{1}, b\right) ; h^{\prime}(x)=p\right\}$ is either the empty set or a nontrivial closed interval for each $p \in P$ since $f_{0}^{\prime}$ is nondecreasing on $\left[x_{1}, b\right)$. We then set

$$
\begin{aligned}
f(x) & =f_{0}\left(x_{1}\right)+\int_{x_{1}}^{\infty} h(z) d z \text { for } x, x_{1} \leq x \leq b \\
& =f_{0}(x)
\end{aligned}
$$

By the choice of $h$ this $f$ is in $C_{P}^{2}(0, b)$ and $f \geq f_{0}$ in $[0, b)$. Since $f=f_{0}$ near $\boldsymbol{x}=0$ (even if $\boldsymbol{x}_{1}=0$ ) the conditions on derivatives are clearly satisfied.

B. Proof of Theorem 6.8. Since $\varphi \in A_{P}(Q)$ implies that $\varphi$ is locally admissible near any $(\hat{t}, \hat{x})$ in $Q$, the 'if' part is trivial. We then prove the 'only if' part. Since the proof for a supersolution parallels that for a subsolution, we present the proof for a subsolution.

Let $u$ be a subsolution of (E). For $(\hat{t}, \hat{x}) \in Q$ let $\varphi \in C(Q)$ be locally admissible near $(\hat{t}, \hat{x})$ in $Q$ with

$$
\max _{Q}\left(u^{*}-\varphi\right)=\left(u^{*}-\varphi\right)(\hat{t}, \hat{x})
$$

Case 1. $\varphi(\hat{t}, \cdot)$ is $P$-faceted at $\hat{\boldsymbol{x}}$. By definition there are bounded open intervals $I$ and $J$ such that $\hat{t} \in I \subset(0, T), R(\varphi(\hat{t}, \cdot), \hat{\boldsymbol{x}}) \subset J \subset \Omega$ and that $\left.\underline{\varphi}\right|_{I+J} \in A_{P}(I \times J)$. Let $I_{1}$ and $J_{1}$ be open intervals such that $\bar{I}_{1} \subset I$ and $\bar{J}_{1} \subset J$ and that $\hat{t} \in I_{1}$ and $R(\varphi(\hat{t}, \cdot), \hat{x}) \subset J_{1}$. By Extension Lemma 6.11 there is $\psi \in A_{P}(Q)$ such that $\varphi \leq \psi$ in $Q$ and $\varphi=\psi$ in $\overline{I_{1}} \times \overline{J_{2}}$. We thus observe that

$$
\max _{Q}\left(u^{*}-\psi\right)=\left(u^{*}-\psi\right)(\hat{t}, \hat{x})
$$

Since $u$ is a subsolution, we see

$$
\hat{\psi}_{t}+F\left(\hat{t}, \hat{\psi}_{x}, \Lambda_{W}(\psi(\hat{t}, \cdot), \hat{x}) \leq 0\right.
$$

with $\hat{\psi}_{t}=\psi_{t}(\hat{t}, \hat{x}), \hat{\psi}_{x}=\psi_{\infty}(\hat{t}, \hat{x})$. Since $\varphi=\psi$ in $\bar{I}_{1} \times \bar{J}_{1}$ and $J_{1}$ contains the faceted region $R(\varphi(\hat{t}, \cdot), \hat{x})$, this inequality is still valid if we replace $\psi$ by $\varphi$. We thus obtain (2.1) for $\varphi$ which we want to prove.

Case 2. $\varphi_{\text {ə }}(\hat{t}, \hat{z}) \notin P$.

The proof parallels Case 1 if we replace $R(\varphi(\hat{t}, \cdot), \hat{x})$ as a single point $\{\hat{x}\}$. 
C. Proof of Theorem 6.9. If $\varphi$ is locally admissible near $(\hat{t}, \hat{x})$ in $Q$, then $\varphi$ is an admissible super function at $(\hat{t}, \hat{x})$ in $Q$ so the 'if' part is trivial. We then prove the 'only if' part. Since the proof for a supersolution parallels that for a subsolution, we present the proof for a subsolution.

Let $u$ be a subsolution in local sense of $(E)$. For $(\hat{t}, \hat{x}) \in Q$ let $\varphi$ be an admissible super function at $(\hat{t}, \hat{x})$ in $Q$ with

$$
\max _{Q}\left(u^{*}-\varphi\right)=\left(u^{*}-\varphi\right)(\hat{t}, \hat{x})
$$

We shall construct a good locally admissible function $\psi$ such that $\psi(\hat{t}, \hat{x})=$ $\varphi(\hat{t}, \hat{x}), \psi \geq \varphi$ in $Q$.

Case 1. $\varphi(\hat{t}, \cdot)$ is $P$-faceted at $\hat{x}$ in $\Omega$ and $\hat{x} \in \operatorname{int} R(\varphi(\hat{t}, \cdot), \hat{x})$.

Step 1. By (A) of $\$ 6.5$ there is $\tau \in \mathcal{T}_{P}^{+} \varphi(\hat{t}, \hat{x})$. By definition there are a modulus $\omega$ and three positive numbers $\delta, \delta_{+}, \delta_{-}$such that

$$
\varphi(t, x)-\varphi(\hat{t}, \hat{x}) \leq \tau(t-\hat{t})+p(x-\hat{x})+\omega(|t-\hat{t}|)|t-\hat{t}|
$$

for all $(t, x) \in Q\left(\delta, \delta_{+}, \delta_{-}\right)=(\hat{t}-\delta, \hat{t}+\delta) \times \tilde{N}^{-1}\left(\varphi(\hat{t}, \cdot), \hat{x}, \delta_{+}, \delta_{-}\right)$, where $p=\varphi_{x}(\hat{t}, \hat{x}) \in P$. As is well known there is $\theta \in C^{1}(\mathbf{R})$ such that $\theta(0)=$ $\theta^{\prime}(0)=0$ and

$$
\omega(|t-\hat{t}|)|t-\hat{t}| \leq \theta(|t-\hat{t}|) .
$$

Although it is elementary but we present a general form of this fact.

6.14. Lemma. Let $\omega$ be a modulus. Then there is $\theta \in C^{k}[0, \infty)$ such that $\theta^{(j)}(0)=0$ and $\theta^{(j)}(x) \geq 0$ for $x \geq 0$ with $0 \leq j \leq k$ and $\omega(|\rho|)|\rho|^{k} \leq \theta(|\rho|)$ for all $\rho \in \mathbf{R}$. In particular for a given $x_{0} \in \mathbf{R}^{m}, G(x)=\theta\left(\left|x-x_{0}\right|\right)$ is $C^{h}$ as a function of $x \in \mathbf{R}^{m}$.

Proof of Lemma 6.14. This is a simple extension of [CEL, Lemma I.4]. We set

$$
\theta_{j}(t)=\int_{t}^{2 t} \theta_{j-1}(s) d s, j \geq 1, \theta_{0}=\omega \text { for } t \geq 0,
$$

so that $\theta_{j} \in C^{j}[0, \infty)$ with $\theta^{(i)}(0)=0$ for $0 \leq i \leq j$. Since $\theta_{j}$ is nondecreasing we have

$$
\theta_{j}(t) \geq t \theta_{j-1}(t) \text { for } t \geq 0
$$

so that $\theta_{j}(t) \geq t^{j} \omega(t)$, we thus observe that $\theta=\theta_{k}$ has all desired properties; $C^{k}$ property of $G$ at $x=x_{0}$ follows from $\theta^{(j)}(0)=0$ for $0 \leq j \leq k$.

By our choice of $\theta$ we have

$$
\varphi(t, x)-\varphi(\hat{t}, \hat{x}) \leq p(x-\hat{x})+g(t) \text { with } g(t)=\tau(t-\hat{t})+\theta(|t-\hat{t}|)
$$

for all $(t, x) \in Q\left(\delta, \delta_{+}, \delta_{-}\right)$. 
Step 2. We shall prove: For sufficiently small $\ell>0$ there is $\xi_{\iota} \in C(\bar{J})$ with $J=\{x ; \operatorname{dist}(x, R(\varphi(\hat{t}, \cdot), \hat{x}))<\ell\}$ such that

(i) $\xi_{l}$ is faceted at $\hat{x}$ in $J$ with $R\left(\xi_{l}, \hat{x}\right) \supset R(\varphi(\hat{t}, \cdot), x)$ and $\xi_{l}=\varphi(\hat{t}, \cdot)$ in $R(\varphi(\hat{t}, \cdot), \hat{x})$;

(ii) $\chi_{+}\left(\xi_{\iota}, \hat{x}\right)=\chi_{+}(\varphi(\hat{t}, \cdot), \hat{z}), \chi_{-}\left(\xi_{\iota}, \hat{x}\right)=\chi-(\varphi(\hat{t}, \cdot), \hat{x})$;

(iii) $\varphi(t, x) \leq \xi_{\ell}(x)+g(t)$ for $(t, x) \in \bar{I} \times \bar{J}$ with some neighborhood $I$ of $\hat{t}$.

This is the essential part of the proof, which shows that our choice of $\tilde{N}^{-1}$ in the definition of $\mathcal{T}_{P}^{+}$is suitable. Let $\left[b_{-}, b_{+}\right]$denote $R(\varphi(\hat{t}, \cdot), \hat{z})$. On $\left(b_{-}, b_{+}\right)$we set $\xi_{l}=\varphi(\hat{t}, \cdot)$. We extend $\xi_{\ell}$ on $\left[b_{-}-\ell, b_{+}+\ell\right]$ in the following way. Since the extension on $\left[b_{-}-\ell, b_{-}\right]$parallels that on $\left[b_{+}, b_{+}+l\right]$ we only show the way to extend $\xi_{\ell}$ on $\left[b_{+}+\ell\right]$. If $\chi_{+}(\varphi(\hat{t}, \cdot), \hat{x})=1$ we take

$$
\begin{array}{r}
\xi_{l}(x)=\sup \{\varphi(t, x)-g(t) ;|t-\hat{t}| \leq \delta / 2\} \\
\text { for } b_{+} \leq x \leq b_{+}+\ell
\end{array}
$$

where $\ell<\delta_{+}$. Since $\varphi(t, \cdot)$ is continuous, $\xi_{\ell}$ is lowersemicontinuous. Since $\varphi$ is uppersemicontinuous, the supremum is attained. Thus we see that $\xi_{l}$ is now continuous in $\left[b_{+}, b_{+}+\ell\right]$. Since $(6.1)$ holds for $(t, x) \in[\hat{t}-\delta / 2, \hat{t}+$ $\delta / 2] \times\left[b_{-}, b_{+}\right]$,

$$
\varphi\left(t, b_{+}\right)-g(t) \leq p\left(b_{+}-\hat{x}\right)+\varphi(\hat{t}, \hat{x})=\varphi\left(\hat{t}, b_{+}\right)
$$

for $t \in[\hat{t}-\delta / 2, \hat{t}+\delta / 2]$. This implies $\xi_{l}\left(b_{+}\right) \leq \varphi\left(\hat{t}, b_{+}\right)$. Since $\xi_{l}\left(b_{+}\right) \geq$ $\varphi\left(\hat{t}, b_{+}\right)$is trivial, we have

$$
\xi_{l}\left(b_{+}\right)=\varphi\left(\hat{t}, b_{+}\right)
$$

which yields the continuity of $\xi_{l}$ at $x=b_{+}$. By definition $\xi_{l}(x) \geq \varphi(\hat{t}, x)$ so that $\xi_{l}$ is a restriction on $\left[b_{-}, b_{+}+\ell\right]$ of some faceted function $\tilde{\xi}$ with $\chi_{+}(\tilde{\xi}, \hat{x})=1$. Since $\chi_{+}(\tilde{\xi}, \hat{x})$ is independent of $\tilde{\xi}$ we safely write $\chi_{+}\left(\xi_{\ell}, \hat{x}\right)$ which is consistent with our original $\chi_{+}$(if $\xi_{\ell}$ is faceted at $\hat{x}$ ). By definition it is clear

$$
\varphi(t, x) \leq \xi_{l}(x)+g(t) \text { on }[\hat{t}-\delta / 2, \hat{t}+\delta / 2] \times\left[b_{-}, b_{+}+\ell\right]
$$

Assume now that $\chi_{+}(\varphi(\hat{t}, \cdot), \hat{x})=-1$. For sufficiently small $\ell>0$ we have

$$
\varphi\left(\hat{t}, b_{+}+\ell\right)<\varphi(\hat{t}, \hat{x})+p(x-\hat{x}) .
$$

Since $\varphi$ is uppersemicontinuous, there is a small $\sigma>0\left(\ell+\sigma<\delta_{+}, \sigma<\right.$ $\ell, \sigma<\delta / 2)$ such that

$$
\varphi(t, x)<\varphi(\hat{t}, \hat{x})+p(x-\hat{x})
$$


for $\left|x-\left(b_{+}+\ell\right)\right| \leq \sigma,|t-\hat{t}| \leq \sigma$. We take a continuous function $\xi_{l}(x)$ for $\left|x-\left(b_{+}+\ell\right)\right| \leq \sigma$ such that

$$
\varphi(t, x)<\xi_{\ell}(x)<\varphi(\hat{t}, \hat{x})+p(x-\hat{x}),\left|x-\left(b_{+}+\ell\right)\right|<\sigma
$$

and that

$$
\xi_{\ell}(x)=\varphi(\hat{t}, \hat{x})+p(x-\hat{x}) \text { at } x=b_{+}+\ell \pm \sigma
$$

If we set

$$
\xi_{\ell}(x)=\varphi(\hat{t}, \hat{x})+p(x-\hat{x}) \text { for } b_{+} \leq x \leq b_{+}+\ell-\sigma
$$

then $\xi_{\ell}$ is continuous in $\left[b_{+}, b_{+}+\ell\right]$ with $\chi_{+}\left(\xi_{\ell}, \hat{x}\right)=-1$. Note that $(6.1)$ hold for

$$
x \in\left[b_{-}, b_{+}+\delta_{+}\right),|t-\hat{t}|<\delta \quad \text { since } \quad \chi_{+}(\varphi(\hat{t}, \cdot), \hat{x})=-1
$$

This yields

$$
\varphi(t, x) \leq \xi_{\ell}(x)+g(t) \text { for } b_{+} \leq x \leq b_{+}+\ell-\sigma,|t-\hat{t}|<\delta .
$$

For $x, b_{+}+\ell-\sigma \leq x \leq b_{+}+\ell$ by the choice by $\xi_{\ell}$ we have

$$
\varphi(t, x)<\xi_{\ell}(x) \text { for }|t-\hat{t}| \leq \sigma .
$$

Thus (iii) holds for $|t-\hat{t}| \leq \sigma, x \in\left[b_{-}, b_{+}+\ell\right]$. Extending $\xi_{\ell}$ on $\left[b_{-}, b_{-}-\ell\right]$ in the same way we obtain $\xi_{\ell}$ satisfying (i), (ii), (iii) of this step.

Step 3. We shall prove: There is $f_{\ell} \in C_{P}^{2}(J) \cap C(\bar{J})$ such that

(i) $f_{l}$ is faceted at $\hat{x}$ in $J$ with $R\left(f_{l}, \hat{x}\right) \subset R\left(\xi_{l}, \hat{x}\right)$;

(ii) $f_{l}=\xi_{\ell}$ on $R\left(f_{\ell}, \hat{x}\right)$ and $f_{\ell}>\xi_{\ell}$ on $\bar{J} \backslash R\left(f_{\ell}, \hat{x}\right)$;

(iii) $\chi_{+}\left(f_{l}, \hat{x}\right)=\chi_{+}\left(\xi_{\ell}, \hat{x}\right), \chi_{-}\left(f_{\ell}, \hat{x}\right)=\chi_{-}\left(\xi_{\ell}, \hat{x}\right)$;

(iv) dist $\left(R\left(f_{\ell}, \hat{x}\right), \partial R\left(\xi_{\ell}, \hat{x}\right)\right) \leq \ell$.

It is not difficult to choose such $f_{\ell}$ if $C_{P}^{2}(J)$ is replaced by $C^{2}(J)$. Indeed we have the next approximation lemma.

6.15. Lemma. Let $\xi \in C(\tilde{J})$ be faceted at $\hat{\boldsymbol{x}}$ in $\tilde{J}$ where $\tilde{J}$ is an open interval. For each $\ell^{\prime}>0$ there is $f \in C^{2}(\tilde{J})$ such that

(i) $f$ is faceted at $\hat{\boldsymbol{x}}$ in $\tilde{J}$ with $R(f, \hat{x}) \subset R(\xi, \hat{x})$;

(ii) $f=\xi$ on $R(f, \hat{x})$ and $f>\xi$ on $\tilde{J} \backslash R(f, \hat{x})$;

(iii) $\chi_{ \pm}(f, \hat{x})=\chi_{ \pm}(\xi, \hat{x})$, respectively;

(iv) dist $(R(f, \hat{x}), \partial R(\xi, x)) \leq \ell^{\prime}$;

(v) $f \leq \xi+\ell^{\prime}$ in $\tilde{J}$; 
(vi) $R(f, \hat{x})=R(\xi, \hat{x})$ if $\chi(\xi, \hat{x})=-1$.

To prove Step 3 we apply this lemma with $\ell^{\prime}=\ell, \xi=\xi_{\iota}$, where $\xi$ is continuously extended in some neighborhood $\tilde{J}$ of $\bar{J}$ and we denote $f$ by $\bar{f}_{\ell}$. Since $\bar{f}_{\ell} \in C^{2}(\tilde{J})$ is faceted at $\hat{x}$, there is a neighborhood $J_{2}$ of $R\left(\bar{f}_{\ell}, \hat{x}\right)$ such that $\left.\bar{f}_{\ell}\right|_{J_{2}} \in C_{P}^{2}\left(J_{2}\right)$ with $\bar{f}_{\ell}^{\prime}(x) \notin P$ for $x \in J_{2} \backslash R\left(\bar{f}_{\ell}, \hat{x}\right)$. We take a neighborhood $J^{\prime}$ of $R\left(\bar{f}_{l}, \hat{x}\right)$ in $J_{2}$ and apply Lemma 6.12 with $f_{0}=\bar{f}_{l}$ and obtain $f \in C_{P}^{2}(J)$ which we denote by $f_{\ell}$. Since $f_{\ell}=\bar{f}_{\ell}$ in $J^{\prime}$ and $f_{\ell} \geq \bar{f}_{\ell}$ in $\tilde{J}$ our $f_{\ell}$ satisfies all properties (i)-(iv) of this step.

Step 4. By Step 2 we observe that

$$
\varphi(t, x) \leq f_{\ell}(x)+g(t),(t, x) \in \bar{I} \times \bar{J} .
$$

We extend $\psi_{\ell}(t, x)=f_{\ell}(x)+g(t)$ outside $\bar{I} \times \bar{J}$ so that $\psi_{\ell} \in C(Q)$ and $\varphi \leq \psi_{\ell}$ in $Q$. This is possible since $\varphi$ is uppersemicontinuous (cf. Lemma 6.18). Since $f_{\ell} \in C_{P}^{2}(J)$ we have $\left.\psi_{\ell}\right|_{I \times J} \in A_{P}(I \times J)$, so that $\psi_{\ell}$ is locally admissible near $(\hat{t}, \hat{x})$ in $Q$. Since $\psi_{\ell}(\hat{t}, \hat{x})=\varphi(\hat{t}, \hat{x})$ and $\varphi \leq \psi_{\ell}$ and since $\varphi$ is a test function of $u$ at $(\hat{t}, \hat{x})$ we have

$$
\max _{Q}\left(u^{*}-\psi_{\ell}\right)=\left(u^{*}-\psi_{\ell}\right)(\hat{t}, \hat{x})
$$

Since $u$ is a subsolution in local sense of (E),

$$
g^{\prime}(\hat{t})+F\left(\hat{t}, f_{\ell}^{\prime}(\hat{x}), \Lambda_{W}\left(f_{\ell}, \hat{x}\right)\right) \leq 0 .
$$

Note that by Step 2 and $3 \chi\left(f_{\ell}, \hat{x}\right)=\chi(\varphi(\hat{t}, \cdot), \hat{x})$ and

$$
\begin{aligned}
& \left|L\left(f_{\ell}, \hat{x}\right)-L(\varphi(\hat{t}, \cdot), \hat{x})\right| \\
& \leq\left|L\left(f_{\ell}, \hat{x}\right)-L\left(\xi_{\ell}, \hat{x}\right)\right|+\left|L\left(\xi_{\ell}, \hat{x}\right)-L(\varphi(\hat{t}, \cdot), \hat{x})\right| \\
& \leq 2 \ell+2 \ell=4 \ell .
\end{aligned}
$$

This implies

$$
\Lambda_{W}\left(f_{\ell}, \hat{x}\right) \rightarrow \Lambda_{W}(\varphi(\hat{t}, \cdot), \hat{x})
$$

as $\ell \rightarrow 0$ since $f_{\ell}^{\prime}(\hat{x})=p$. By the continuity (F1) of $F$ we send $\ell \rightarrow 0$ in (6.2) to get

$$
\tau+F\left(\hat{t}, p, \Lambda_{W}(\varphi(\hat{t}, \cdot), \hat{x})\right) \leq 0
$$

since $g^{\prime}(\hat{t})=\tau$. We have thus proved (i) in $\S 6.6$ for Case 1 .

Case 2. There is $(\tau, p, X) \in \mathcal{P}^{+} \varphi(\hat{t}, \hat{x})$ with $p \notin P$.

By definition of $\mathcal{P}^{+}$there is a modulus $\omega$ such that

$$
\begin{aligned}
\varphi(t, x) & -\varphi(\hat{t}, \hat{x}) \leq \tau(t-\hat{t})+p(x-\hat{x})+\frac{1}{2} X(x-\hat{x})^{2} \\
& +\omega\left(|t-\hat{t}|+|x-x|^{2}\right)\left(|t-\hat{t}|+|x-\hat{x}|^{2}\right) \text { in } Q .
\end{aligned}
$$


The last term is dominated by

$$
\begin{aligned}
& 2 \omega(2|t-\hat{t}|)|t-\hat{t}| \text { if }|t-\hat{t}| \geq|x-\hat{x}|^{2} \\
& 2 \omega\left(2|x-\hat{x}|^{2}\right)|x-\hat{x}|^{2} \text { if }|t-\hat{t}| \leq|x-\hat{x}|^{2}
\end{aligned}
$$

so

$$
\begin{aligned}
& \omega\left(|t-\hat{t}|+|x-\hat{x}|^{2}\right)\left(|t-\hat{t}|+|x-\hat{x}|^{2}\right) \\
& \leq \omega_{1}(|t-\hat{t}|)|t-\hat{t}|+\omega_{2}(|x-\hat{x}|)|x-\hat{x}|^{2}
\end{aligned}
$$

with another modulus $\omega_{1}(\rho)=2 \omega(2 \rho), \omega_{2}(\rho)=2 \omega\left(2 \rho^{2}\right)$. By Lemma 6.14 there are $\theta_{1} \in C^{1}[0, \infty), \theta_{2} \in C^{2}[0, \infty)$ such that $\theta_{1}(0)=\theta_{1}^{\prime}(0)=0, \theta_{2}(0)=$ $\theta_{2}^{\prime}(0)=\theta_{2}^{\prime \prime}(0)=0$ and that

$$
\begin{aligned}
& \omega_{1}(|t-\hat{t}|)|t-\hat{t}| \leq \theta_{1}(|t-\hat{t}|) \\
& \omega_{2}(|x-\hat{x}|)|x-\hat{x}|^{2} \leq \theta_{2}(|x-\hat{x}|)
\end{aligned}
$$

We have thus observed

$$
\begin{aligned}
\varphi(t, x)-\varphi(\hat{t}, \hat{x}) & \leq \tau(t-\hat{t})+p(x-\hat{x})+\frac{1}{2} X(x-\hat{x})^{2} \\
& +\theta_{1}(|t-\hat{t}|)+\theta_{2}(|x-\hat{x}|) \text { in } Q
\end{aligned}
$$

We then set

$$
\begin{aligned}
& f(x)=\varphi(\hat{t}, \hat{x})+p(x-\hat{x})+\frac{1}{2} X(x-\hat{x})^{2}+\theta_{2}(|x-\hat{x}|), \\
& g(t)=\tau(t-\hat{t})+\theta_{1}(|t-\hat{t}|), \psi(t, x)=f(x)+g(t)
\end{aligned}
$$

so that $f \in C^{2}(\mathbf{R}), g \in C^{1}(\mathbf{R})$ and

$$
\varphi(\hat{t}, \hat{x})=\psi(\hat{t}, \hat{x}), \varphi \leq \psi \quad \text { in } Q
$$

This yields

$$
\max _{Q}\left(u^{*}-\psi\right)=\left(u^{*}-\psi\right)(\hat{t}, \hat{x}),
$$

since $\varphi$ is a test function of $u$ at $(\hat{t}, \hat{x})$. By definition of $f$ and $g$ we have

$$
\tau=g^{\prime}(\hat{t}), p=f^{\prime}(\hat{z}) \notin P, X=f^{\prime \prime}(\hat{z}) .
$$

Since $p \notin P, f \in C^{2}(\mathbf{R})$ and $g \in C^{1}(R), \psi$ is locally admissible near $(\hat{t}, \hat{z})$ in $Q$. Since $u$ is a subsolution in local sense of $(E)$ we have

$$
\tau+F\left(\hat{t}, p, W^{\prime \prime}(p) X\right) \leq 0
$$


which prove (ii) in $\$ 6.6$ for Case 2.

Case 3. $\varphi(\hat{t}, \cdot)$ is $P$-faceted at $\hat{\boldsymbol{x}}$ in $\Omega$ but $\hat{\boldsymbol{x}} \in \partial R(\varphi(\hat{t}, \cdot), \hat{\boldsymbol{x}})$.

In this case $\varphi$ is locally admissible near $(\hat{t}, \hat{x})$ in $Q$ by (C) of $\S 6.5$. Since $u$ is a subsolution in local sense of (E) it is clear (iii) of $\$ 6.6$ holds. The proof of Theorem 6.9 is now complete.

6.16. Remark. We do not use degenerate ellipticity (F2) and (F3) in proving Theorem 6.8 and 6.9. We are forced to use approximation argument so the continuity (F1) is invoked. In Theorem 6.8 we do not need even (F1); all we need is that $F$ is a function from $[0, T) \times \mathbf{R} \times \mathbf{R}$ to $\mathbf{R}$.

6.17. Remark (Definition of solutions). In Definition 6.7 for local version our test function $\psi$ at $(\hat{t}, \hat{x})$ is assumed to be in $C(Q)$. It turns out that we may weaken this requirement so that $\psi \in C\left(Q^{\prime}\right)$ is locally admissible at $(\hat{t}, \hat{x})$ in $Q^{\prime}$, where $Q^{\prime}$ is some rectangular neighborhood of $(\hat{t}, \hat{x})$ in $Q$ and that

$$
\max _{Q^{\prime}}\left(u^{*}-\psi\right)=\left(u^{*}-\psi\right)(\hat{t}, \hat{x})
$$

To see this let $\hat{Q}$ be a rectangular neighborhood of $(\hat{t}, \hat{x})$ in $Q^{\prime}$ with $\left.\psi\right|_{\hat{Q}} \in$ $A_{\underline{p}}(\hat{Q})$. It suffices to prove that there is $\bar{\psi} \in C(Q)$ that satisfies

(i) $\psi=\bar{\psi}$ in $\hat{Q}$,

(ii) $\psi \leq \bar{\psi}$ in $Q^{\prime}$,

(iii) $\max _{Q^{\prime}}\left(u^{*}-\psi\right)=\max _{Q}\left(u^{*}-\bar{\psi}\right)$.

We may assume that $\max _{Q^{\prime}}\left(u^{*}-\psi\right)=0$ and $u^{*}<\psi$ near $\partial Q^{\prime}$. We may also assume $\psi \in C(\bar{Q})$ by taking $Q^{\prime}$ a little bit smaller. Our desired $\psi$ is constructed by using the next lemma.

6.18. Lemma. Let $w$ be an uppersemicontinuous function in $Q \backslash Q^{\prime}$ with values in $[-\infty, \infty)$. Let $\psi \in C\left(\partial Q^{\prime}\right)$ satisfy $w<\psi$ on $\partial Q^{\prime}$. Then there is $\bar{\psi} \in C\left(Q \backslash Q^{\prime}\right)$ that satisfies $w \leq \bar{\psi}$ in $Q \backslash Q^{\prime}$ and $\bar{\psi}=\psi$ on $\partial Q^{\prime}$.

Proof. As in the proof of Lemma 6.11 we take $d$ and $\bar{d}$ and define $J^{r}=J(r)$ and $I^{r}=I(r)$ so that $I^{0} \times J^{0}=Q^{\prime}$ and that $U_{r \geq 0} I^{r}=(0, T), \cup_{r \geq 0} J^{r}=\Omega$. We set

$$
h(r)=\max \left\{w(t, x),(t, x) \in Q \backslash Q^{\prime},(t, x) \in \vec{I} \times \vec{J}\right\} .
$$

Since $\vec{I} \times \vec{J}$ is compact and $w$ is uppersemicontinuous with $w<\infty$ on $Q$ we see $h$ is uppersemicontinuous in $[0, \infty)$. Since $h$ is nondecreasing, there is a continuous function $\bar{h} \geq h$ with $h(0)=\bar{h}(0)$. We then set

$$
\psi_{0}(t, x)=\bar{h}\left(\max (d(x), \bar{d}(t)) \text { for }(t, x) \in Q \backslash Q^{\prime},\right.
$$

so that $w \leq \psi_{0}$ in $Q \backslash Q^{\prime}$. Since $\sup _{\partial Q^{\prime}} w<\psi$, we modify $\psi_{0}$ near $\partial Q^{\prime}$ to obtain $\bar{\psi}$ satisfying $\bar{\psi} \geq w$ in $Q \backslash Q^{\prime}$ and $\bar{\psi}=\psi$ on $\partial Q^{\prime}$. 
From Remark 6.17 (with Theorem 6.8) it easily follows that a restriction of subsolution is a subsolution.

6.19. Proposition (Restrictions). Let $u$ be a subsolution (supersolution) of $(E)$ in $Q$. Let $Q_{0}=I_{0} \times J_{0}$ be an open rectangular set in $Q_{0}$. Then $\left.u\right|_{Q_{0}}$ is a subsolution (supersolution) of (E) in $Q_{0}$.

6.20. Proposition. Let $\left\{Q_{r}\right\}_{r=1}^{\infty}$ be a sequence of rectangular domain exhausting $Q$, i.e. $Q_{r} \subset Q_{r+1}$ and $\cup_{r=1}^{\infty} Q_{r}=Q$. For a function $u$ on $Q$ assume that $u$ is a subsolution (supersolution) of $(E)$ on each $Q_{r}$. Then $u$ is a subsolution (supersolution) in $Q$ provided that $u^{*}<\infty\left(u_{*}>-\infty\right)$ on $[0, T) \times \bar{\Omega}$.

Tha last statement trivially follows from definition of a subsolution in $\$ 2$.

\section{§7. Proof of Comparison Theorems.}

The basic strategy is in finding suitable test functions of $u$ and $v$ to obtain a contradiction by assuming that the conclusion $u^{*} \leq v_{*}$ were false. We use the method developed in [CGG] and [Go]. However, for example if $u$ and $v$ are faceted at the points which we are interested, the standard maximum principle [CIL] does not apply. We apply our maximum principle to overcome this difficulty. Unfortunately, $u$ and $v$ are not necessarily faceted, so we need to use sup-convolution to regularize these functions. Such regularization is used in proving the standard maximum principle; however, the convolution is different from the usual one.

For $z=(t, x), z^{\prime}=(s, y) \in Q=(0, T) \times \Omega$ we set

$$
w\left(z, z^{\prime}\right)=u(z)-v\left(z^{\prime}\right)
$$

We consider "barrier functions"

$$
\begin{aligned}
& \bar{\Psi}_{\zeta}\left(z, z^{\prime} ; \epsilon, \sigma, \gamma, \gamma^{\prime}\right)=B_{\epsilon}(x-y-\zeta)+S\left(t, s ; \sigma, \gamma, \gamma^{\prime}\right), \\
& B_{\epsilon}(x)=x^{2} / \epsilon, S\left(t, s ; \sigma, \gamma, \gamma^{\prime}\right)=B_{\sigma}(t-s)+\gamma /(T-t)+\gamma^{\prime} /(T-s)
\end{aligned}
$$

for positive parameters $\epsilon, \sigma, \gamma, \gamma^{\prime}$ and a real parameter $\zeta$. The term $S$ is very large near $t=T$ or $s=T$ while $B_{\epsilon}(x-y-\zeta)$ (resp. $B_{\sigma}(t-s)$ ) is very large away from $x-y=\zeta$ (resp. $t=s$ ) if $\epsilon$ (resp. $\sigma$ ) is sufficiently small. We often write $\Psi_{\zeta}\left(z, z^{\prime}\right)$ and $S(t, s)$ instead of writing dependence of all positive parameters. As usual we shall analyse maximizers of

$$
\Phi_{\zeta}\left(z, z^{\prime}\right)=w\left(z, z^{\prime}\right)-\Psi_{\zeta}\left(z, z^{\prime}\right)
$$

In proving the Comparison Theorem we may assume that $u$ and $-v$ are uppersemicontinuous in $\bar{Q}$ with value in $R \cup\{-\infty\}$ by considering $u^{*}$ and $v_{*}$ 
instead of $u$ and $v$. For this reason we shall always assume this property for $u$ and $v$ in this section. Since $\bar{Q}$ is compact, there is a maximizer $\left(z_{\zeta}, z_{\zeta}^{\prime}\right)$ in $\bar{Q} \times \bar{Q}$ of $\Phi_{\zeta}$ over $\bar{Q} \times \bar{Q}$ i.e.,

$$
\sup \Phi_{\zeta} \equiv \sup \left\{\Phi_{\zeta}\left(z, z^{\prime}\right) ;\left(z, z^{\prime}\right) \in \bar{Q} \times \bar{Q}\right\}=\Phi_{\zeta}\left(z_{\zeta}, z_{\zeta}^{\prime}\right)
$$

Before going into the detail we summarize our method. We may assume that $F$ is continuous up to $t=T$ and $u^{*} \leq v_{*}$ on $\bar{\partial}_{p} Q\left(=\overline{\partial_{p} Q}=[0, T] \times \partial \Omega \times\right.$ $\{0\} \times \bar{\Omega})$ by taking $T$ smaller than original $T$.

We first show that $\Phi_{\zeta}$ takes a positive maximum only on $Q \times Q$ by taking all parameters sufficiently small. This follows from the order of $u$ and $v$ on the parabolic boundary. The argument is standard for $\zeta=0$. We state a quantitative version for later use (cf. §A. Choice of parameters.)

We then classify the situations depending on the derivative of $\Psi_{\zeta}$ at a maximizer of $\Phi_{\zeta}(\mathrm{cf}$. $\S \mathrm{B})$. To simplify the explanation we consider the case $P=\{0\}$. If there is a sequence $\zeta_{j} \rightarrow 0$ such that the (spatial) derivative of $\Psi_{\zeta_{j}}$ at some maximizer of $\Phi_{\zeta_{j}}$ does not equal zero, then the standard maximum principle [CIL] does apply to get a contradiction. (cf. §G). We must analyse the remaining case (Case I), i.e., the derivative of $\Psi_{6}$ always equal zero at any maximizer $\left(\hat{t}_{\zeta}, \hat{x}_{\zeta}, \hat{s}_{\zeta}, \hat{y}_{\zeta}\right)$ for small $\zeta$, which yields $B_{\epsilon}^{\prime}\left(\hat{x}_{\zeta}-\right.$ $\left.\hat{y}_{\zeta}-\zeta\right)=0$ (cf. $\left.\S C\right)$. This implies that $g(\zeta)=\sup \Phi_{\zeta}$ is constant for small $\zeta$ (Constancy Lemma 7.5). From this property we see $u\left(\hat{t}_{0}, \cdot\right)$ and $-v\left(\hat{\boldsymbol{s}}_{0}, \cdot\right)$ take their local maximums at $\hat{\boldsymbol{x}}_{0}$ and $\hat{y}_{0}$ respectively (Proposition 7.4).

In general $P$ is not a singleton $B_{\epsilon}^{\prime}\left(\hat{x}_{\zeta}-\hat{y}_{\zeta}-\zeta\right)$ is not constant as $\zeta \rightarrow 0$. Fortunately we find some $\zeta_{0}$ (close to zero) such that $B_{\epsilon}^{\prime}\left(\hat{x}_{\zeta}-\hat{y}_{\zeta}-\zeta\right)$ is some constant $p_{0} \in P$ for $\zeta$ close to $\zeta_{0}$ (Lemma 7.4). The local behavior of $u$ and $v$ should be modified as in Proposition 7.4 so several extra efforts are necessary (cf. §C).

We continue to discuss the case $P=\{0\}$. The constancy of $g$ implies

$$
u(t, x)-v(s, y)-S(t, s) \leq u\left(\hat{t}_{0}, \hat{x}_{0}\right)-v\left(\hat{s}_{0}, \hat{y}_{0}\right)-S\left(\hat{t}_{0}, \hat{s}_{0}\right)
$$

for $t, s \in(0, T)$ and $x$ close to $y$, say $|x-y|<\delta$. If $u\left(\hat{t}_{0}, \cdot\right)$ and $v\left(\hat{s}_{0}, \cdot\right)$ are faceted with slope zero with facet length $<\delta / 2$ at $\hat{x}_{0}$ and $\hat{y}_{0}$, respectively, this inequality with $t=\hat{t}_{0}, s=\hat{s}_{0}$ implies that $\chi_{u} \leq 0, \chi_{v} \geq 0$, so that

$$
\chi_{u} / L_{u}-\chi_{v} / L_{v} \leq 0
$$

with

$$
\begin{array}{ll}
\chi_{u}=\chi\left(u\left(\hat{t}_{0}, \cdot\right), \hat{x}_{0}\right), & L_{u}=L\left(u\left(\hat{t}_{0}, \cdot\right), \hat{x}_{0}\right) \\
\chi_{v}=\chi\left(v\left(\hat{\boldsymbol{s}}_{0}, \cdot\right), \hat{y}_{0}\right), & L_{v}=L\left(v\left(\hat{\boldsymbol{s}}_{0}, \cdot\right), \hat{y}_{0}\right) .
\end{array}
$$


Applying the infinitesimal version of the definition of solutions, we get from the above inequality for $u, v$ and $S$ that

$$
\begin{aligned}
& S_{t}\left(\hat{t}_{0}, \hat{s}_{0}\right)+F\left(\hat{t}_{0}, 0, \frac{\chi_{u}}{L_{u}} \Delta\right) \leq 0, \\
& S_{s}\left(\hat{t}_{0}, \hat{s}_{0}\right)-F\left(\hat{s}_{0}, 0, \frac{\chi_{v}}{L_{v}} \Delta\right) \leq 0,
\end{aligned}
$$

where $\Delta=W^{\prime}(+0)-W^{\prime}(-0)$. Adding the last two in equalities with $\chi_{u} / L_{u}-$ $\chi_{v} / L_{v} \leq 0$ and (F2), we have

$$
\frac{\gamma}{\left(T-\hat{t}_{0}\right)^{2}}+\frac{\gamma^{\prime}}{\left(T-\hat{s}_{0}\right)^{2}}+F\left(\hat{t}_{0}, 0, \frac{\chi_{u}}{L_{u}} \Delta\right)-F\left(\hat{s}_{0}, 0, \frac{\chi_{u}}{L_{u}} \Delta\right) \leq 0
$$

yielding

$$
\left(\gamma+\gamma^{\prime}\right) / T^{2} \leq \omega\left(\left|\hat{t}_{0}-\hat{\boldsymbol{s}}_{0}\right|\right)
$$

where $\omega$ is a modules of continuity of $F(t, 0, X)$ in $t$ where uniform continuity (F3) is invoked if $F$ depends on $t$. We fix $\gamma$ and $\gamma^{\prime}$ and letting $\sigma \rightarrow 0$ so that $\hat{t}_{0}-\hat{s}_{0} \rightarrow 0$ (Proposition 7.1). By continuity of $C$ (up to $t=T$ ) we end up with $\left(\gamma+\gamma^{\prime}\right) / T^{2} \leq 0$, a contradiction(cf. $\left.\S \mathrm{F}\right)$.

Unfortunately, $u$ and $v$ are not necessarily faceted so we need to take sup-convolutions with faceted functions (cf. $\S D$ ). If $u\left(\hat{t}_{0}, \cdot\right)$ takes a local maximum at $\hat{x}_{0}$, the sup-convolution $u^{\alpha}\left(\hat{t}_{0}, \cdot\right)$ is faceted in $\mathbf{R}$ at $\hat{x}_{0}$ as we observed in section 5 . The length of facet may be very long. We should apply our maximum principle in section 4 to get $\chi_{u} / L_{u}-\chi_{v} / L_{v} \leq 0$ (cf. $\S \mathrm{E})$. Moreover, we should be careful about the definition of solutions in the infinitesimal version as in section 6 .

The proof when $u$ and $v$ are spatially periodic is easier. We shall remark necessary alternations at the end of this section(cf. $\S \mathrm{H}$ ).

A. Choice of parameters.

7.1. Proposition. Assume that $u$ and $-v$ are uppersemicontinuous in $\bar{Q}$ with values in $\mathbf{R} \cup\{-\infty\}$. Assume that

$$
m_{0}=\sup \{u(z)-v(z) ; z \in Q\}>0 .
$$

(i)For each $m_{0}^{\prime}\left(0<m_{0}^{\prime}<m_{0}\right)$ there are $\gamma_{0}, \gamma_{0}^{\prime}>0$ such that

$$
\sup \Phi_{\zeta}>m_{0}^{\prime} \text { for all } \epsilon>0, \sigma>0, \gamma_{0}>\gamma>0, \gamma_{0}^{\prime}>\gamma^{\prime}>0
$$

and $|\zeta| \leq \kappa_{0}(\epsilon)=\left(\epsilon\left(m_{0}-m_{0}^{\prime}\right)\right)^{1 / 2} / 2$.

(ii)Let $\left(z_{\zeta}, z_{\zeta}^{\prime}\right)=\left(t_{\zeta}, x_{\zeta},{ }_{\zeta \zeta}, y_{\zeta}\right)$ be a maximizer of $\Phi_{\zeta}$ over $\bar{Q} \times \bar{Q}$. Then,

$$
\left|t_{\zeta}-s_{\zeta}\right| \leq(M \sigma)^{1 / 2}, \quad\left|x_{\zeta}-y_{\zeta}-\zeta\right| \leq(M \epsilon)^{1 / 2}
$$


with

$$
M=\sup \left\{w\left(z, z^{\prime}\right),\left(z, z^{\prime}\right) \in \bar{Q} \times \bar{Q}\right\}
$$

for all $\epsilon>0, \sigma>0, \gamma_{0}>\gamma>0, \gamma_{0}^{\prime}>\gamma^{\prime}>0$ and $\zeta$ with $|\zeta| \leq \kappa_{0}(\epsilon)$. In particular,

$$
\lim _{\sigma \rightarrow 0}\left|t_{\zeta}-s_{\zeta}\right|=0, \quad \lim _{\epsilon \rightarrow 0}\left|x_{\zeta}-y_{\zeta}\right|=0 .
$$

(iii) Assume that $u \leq v$ on $\bar{\partial}_{p} Q\left(=\overline{\partial_{p} Q}\right)$ and that $\Omega$ is a bounded open interval. Then there are $\epsilon_{0}, \sigma_{0}$ such that $\left(z_{\zeta}, z_{\zeta}^{\prime}\right)$ is an (interior) point of $Q \times Q$ for all $0<\epsilon<\epsilon_{0}, 0<\sigma<\sigma_{0}, 0<\gamma<\gamma_{0}, 0<\gamma^{\prime}<\gamma_{0}^{\prime}$ and $|\zeta| \leq \kappa_{0}(\epsilon)$.

7.2. Remark. Since $w$ is uppersemicontinuous, we may assume in (iii) that for each $\xi>0$

$$
w\left(z, z^{\prime}\right) \leq \xi, \quad z \in \bar{\partial}_{p} Q \quad \text { or } \quad z^{\prime} \in \bar{\partial}_{p} Q
$$

for all $|x-y|<\left(M \epsilon_{0}\right)^{1 / 2}+\kappa_{0}\left(\epsilon_{0}\right),|t-s|<\left(M \sigma_{0}\right)^{1 / 2}$ with $z=(t, x), z^{\prime}=$ $(s, y)$. In what follows we shall assume $m_{0}>0$ with $\xi=m_{0} / 4, m_{0}^{\prime}=m_{0}-\xi / 2$ and fix $\epsilon_{0}, \sigma_{0}, \gamma_{0}, \gamma_{0}^{\prime}$ so that all properties (i)-(iii) and those in Remark 7.2 hold.

Proof of Proposition 7.1. The proof is standard especially for $\zeta=0$ e.g. [GGIS] but we give it for completeness.

(i) For each $m, m_{0}^{\prime}<m<m_{0}$ there is a point $z_{0}=\left(t_{0}, x_{0}\right) \in Q$ satisfying

$$
m_{0} \geq u\left(z_{0}\right)-v\left(z_{0}\right) \geq m \text {. }
$$

By definition we see

$$
\sup \Phi_{\zeta} \geq \Phi_{\zeta}\left(z_{0}, z_{0}\right) \geq m-\zeta^{2} / \epsilon-S\left(t_{0}, t_{0} ; \sigma, \gamma, \gamma^{\prime}\right)
$$

Take $\gamma_{0}, \gamma_{0}^{\prime}$ small so that

$$
S\left(t_{0}, t_{0} ; \sigma, \gamma_{0}, \gamma_{0}^{\prime}\right)=\gamma_{0} /\left(T-t_{0}\right)+\gamma_{0}^{\prime} /\left(T-t_{0}\right)<\left(m-m_{0}^{\prime}\right) / 2 .
$$

If $m$ is close to $m_{0}$, say $m_{0}-m \leq m-m_{0}^{\prime}$, then $\kappa_{0}(\epsilon)^{2} \leq \epsilon\left(m-m_{0}^{\prime}\right) / 2$. For this choice of $m$, we end up with

$$
\sup \Phi_{\zeta} \geq m-\left(m-m_{0}^{\prime}\right) / 2-\left(m-m_{0}^{\prime}\right) / 2>m_{0}^{\prime}
$$

for $\gamma<\gamma_{0}, \gamma^{\prime}<\gamma_{0}^{\prime}$ provided that $|\zeta| \leq \kappa_{0}(\epsilon)$.

(ii) By $\sup \Phi_{\zeta} \geq m_{0}^{\prime} \geq 0$ we see $w \geq \Psi_{\zeta}$ at $\left(z_{\zeta}, z_{\zeta}^{\prime}\right)$. Since $w$ is uppersemicontinuous, $w$ is bounded on $\bar{Q} \times \bar{Q}$, say $w \leq M$. The inequality $w \geq \Psi_{\zeta}$ at $\left(z_{\zeta}, z_{\zeta}^{\prime}\right)$ yields

$$
\left|x_{\zeta}-y_{\zeta}-\zeta\right|^{2} / \epsilon \leq M \text { and }\left|t_{\zeta}-s_{\zeta}\right|^{2} / \sigma \leq M
$$


or

$$
\left|x_{\zeta}-y_{\zeta}\right| \leq(M \epsilon)^{1 / 2}+|\zeta| \text { and }\left|t_{\zeta}-s_{\zeta}\right| \leq(M \sigma)^{1 / 2} \text {. }
$$

Since $M$ is independent of all parameters $\epsilon, \sigma, \gamma, \gamma^{\prime}$ and $\xi$, this yields (ii).

(iii) We argue by a contradiction. For each $0<\gamma<\gamma_{0}, 0<\gamma^{\prime}<\gamma_{0}^{\prime}$ there were a sequence $\left\{\left(\epsilon_{j}, \sigma_{j}\right)\right\}_{j=1}^{\infty}$ with $\epsilon_{j} \downarrow 0, \sigma_{j} \downarrow 0$ such that there is a maximizer

$$
\left(z_{j}, z_{j}^{\prime}\right) \in(\bar{Q} \times \bar{Q}) \backslash(Q \times Q)
$$

of $\Phi_{\zeta_{j}}\left(\cdot, ; ; \epsilon_{j}, \sigma_{j}, \gamma, \gamma^{\prime}\right)$ over $\bar{Q} \times \bar{Q}$ with some $\zeta_{j},\left|\zeta_{j}\right| \leq \kappa_{0}\left(\epsilon_{j}\right)$. Since $\bar{Q} \times \bar{Q}$ is compact we may assume $z_{j} \rightarrow \bar{z}, z_{j}^{\prime} \rightarrow \vec{z}^{\prime}$ for some point $\bar{z}, \vec{z} \in \bar{Q}$ by taking a subsequence if necessary. By (ii) we observe that $\bar{z}=\vec{z}$.

The condition $\left(z_{j}, z_{j}^{\prime}\right) \notin Q \times Q$ implies that either $z_{j} \in \bar{\delta}_{p} Q$ or $z_{j}^{\prime} \in$ $\bar{\partial}_{p} Q$ since the time component of $z_{j}$ and $z_{j}^{\prime}$ cannot be $T$ because of terms $\gamma /(T-t), \gamma^{\prime} /(T-s)$ in the definition of $\Psi_{\xi}$. Since $\bar{\partial}_{p} Q$ is closed, $\bar{z}$ belongs to $\bar{\partial}_{p} Q$. Since $\left(z_{j}, z_{j}^{\prime}\right)=\left(t_{j}, x_{j}, s_{j}, y_{j}\right)$ is a maximizer of $\Phi_{\zeta},(i)$ implies

$$
0<\frac{m_{0}}{2}<w\left(t_{j}, x_{j}, s_{j}, y_{j}\right)-B_{\epsilon_{j}}\left(x_{j}-y_{j}-\zeta_{j}\right)-S\left(t_{j}, s_{j} ; \sigma_{j}, \gamma, \gamma^{\prime}\right) .
$$

By the monotonicity dependence of the parameters $\epsilon, \sigma$ we see the right hand side is dominated from above by

$$
w\left(t_{j}, x_{j}, s_{j}, y_{j}\right)-B_{\epsilon_{j 0}}\left(x_{j}-y_{j}-\zeta_{j}\right)-B_{\sigma_{j 0}}\left(t_{j}-s_{j}\right)
$$

if $j \geq j_{0}$. Since $w$ is uppersemicontinuous, sending $j \rightarrow \infty$ yields

$$
0<\frac{m_{0}}{2} \leq w(\bar{z}, \bar{z})=u(\bar{z})-v(\bar{z}) .
$$

Since $\bar{z} \in \bar{\partial}_{p} Q$ this contradicts $u \leq v$ on $\bar{\partial}_{p} Q$.

\section{B. Classification.}

We shall divide the situations depending on the value of derivative of $\Psi_{\zeta}$ at a maximizer of $\Phi_{\zeta}$. Let $g$ denote the maximum value of $\Phi_{\zeta}$, i.e.,

$$
g(\zeta)=\sup \Phi_{\zeta}=\sup \left\{\Phi_{\zeta}\left(z, z^{\prime}\right) ;\left(z, z^{\prime}\right) \in \bar{Q} \times \bar{Q}\right\} .
$$

Let $\mathcal{A}(\zeta)$ denote the set of maximizers of $\Phi_{\zeta}$ over $\bar{Q} \times \bar{Q}$, i.e.,

$$
\mathcal{A}(\zeta)=\left\{\left(z, z^{\prime}\right) \in \bar{Q} \times \bar{Q} ; g(\zeta)=\Phi_{\zeta}\left(z, z^{\prime}\right)\right\}
$$

Let $\mathcal{B}(\zeta)$ denote the set of values of derivatives $B_{\varepsilon}(x-y-\zeta)$ at a point of $\mathcal{A}(\zeta)$, i.e.,

$$
\mathcal{B}(\zeta)=\{2(x-y-\zeta) / \epsilon ;(t, x, s, y) \in \mathcal{A}(\zeta)\}
$$

Of course, both $\mathcal{A}$ and $\mathcal{B}$ depend on $\epsilon, \sigma, \gamma, \gamma^{\prime}$ with $0<\epsilon<\epsilon_{0}, 0<\sigma<$ $\sigma_{0}, 0<\gamma<\gamma_{0}, 0<\gamma^{\prime}<\gamma_{0}^{\prime}$; however, we do not write its dependence since we shall fix these numbers in $\S B-\mathrm{E}$. We recall basic properties of $\mathcal{A}$ and $\mathcal{B}$. 
7.3. Proposition on maximizers.

(i) The set $\mathcal{A}(\zeta)$ is a nonempty subset of $Q \times Q$ for $\zeta,|\zeta| \leq \kappa_{0}(\epsilon)$.

(ii) The graph of $\mathcal{A}$ (as a set valued function) is compact, i.e.,

$$
\operatorname{graph} \mathcal{A}=\left\{\left(\zeta, z, z^{\prime}\right) ;\left(z, z^{\prime}\right) \in \mathcal{A}(\zeta),|\zeta| \leq \kappa_{0}(\epsilon)\right\}
$$

is compact in $\left[-\kappa_{0}(\epsilon), \kappa_{0}(\epsilon)\right] \times Q \times Q$.

(iii) The graph of $\mathcal{B}$ is compact in $\left[-\kappa_{0}(\epsilon), \kappa_{0}(\epsilon)\right] \times \mathbf{R}$.

Proof (i) Since $w$ is an uppersemicontinuous function and $\bar{Q}$ is compact, $\mathcal{A}(\zeta)$ is nonempty. The assertion that $\mathcal{A}(\zeta)$ is contained in $Q \times Q$ has been proved in Proposition 7.1 (iv).

(ii) We note that $\Phi_{\zeta}$ is continuous in $\zeta$. Since a supremum of a set of continuous functions is lowersemicontinuous, $g$ is lowersemicontinuous so that $\Phi_{\zeta}-g$ is uppersemicontinuous. The set of maximizers of $\Phi_{\zeta}-g$ in $\Sigma=$ $\left[-\kappa_{0}(\epsilon), \kappa_{0}(\epsilon)\right] \times \bar{Q} \times \bar{Q}$ equals graph $\mathcal{A}$. Since $\Phi_{\zeta}-g$ is uppersemicontinuous on $\Sigma$, graph $\mathcal{A}$ is compact.

(ii) Since graph $\mathcal{B}$ is interpreted as the image of graph $\mathcal{A}$ of a linear mapping, graph $\mathcal{B}$ should be compact.

The situation is divided into two cases.

Case I. There is $\kappa_{1} \leq \kappa_{0}(\epsilon)$ such that $\mathcal{B}(\zeta)$ is contained in $P$ for all $\zeta,|\zeta| \leq \kappa_{1}$. Case II. The negation of Case $I$, that is to say, there is a sequence $\zeta_{j} \rightarrow 0$ such that $\mathcal{B}\left(\zeta_{j}\right)$ is not contained in $P$.

We shall study Case $I$ in $\S C-F$. Our maximum principle for faceted functions will be invoked in $\S \mathrm{E}$.

\section{Local behaviour of $u$ and $v$.}

The next lemma is trivial if $P$ is a singleton.

7.4. Lemma. Consider Case I. For $0<\kappa \leq \kappa_{1}$ there are $\zeta_{0}$ with $\left|\zeta_{0}\right|<$ $\kappa, \delta>0$ with $\delta+\left|\zeta_{0}\right|<\kappa$ and $p_{0} \in P$ such that $\mathcal{B}(\zeta)$ contains $p_{0}$ for all $\zeta$ such that $\left|\zeta-\zeta_{0}\right| \leq \delta,|\zeta| \leq \kappa$.

Proof. Since $P$ is discrete and since graph $\mathcal{B}$ is closed, for each $p \in P$ the set

$$
Y_{p}=\{\zeta ;|\zeta| \leq \kappa, p \in \mathcal{B}(\zeta)\}
$$

is closed in $[-\kappa, \kappa]$. We know that $\mathcal{B}(\zeta)$ is a nonempty subset of $P$, that is to say

$$
[-\kappa, \kappa]=\cup\left\{Y_{p} ; p \in P\right\} .
$$

Since $P$ is at most countable, the Baire category theorem [Y. Chapter 0] says that $Y_{p_{0}}$ contains an interior point $\zeta_{0}$ for some $p_{0} \in P$.

We shall claim that $g(\zeta)-p_{0}\left(\zeta-\zeta_{0}\right)$ is constant on $\left(\zeta_{0}-\delta, \zeta_{0}+\delta\right)$. The next lemma is general and it does not need assumptions we so far assumed. 
7.5. Constancy Lemma. Let $K$ be a compact set in $\mathbf{R}^{N}$ and let $h$ be a real valued uppersemicontinuous function on $K$. Let $\phi$ be a $C^{2}$ function on $\mathbf{R}^{d}$ with $1 \leq d<N$. Let $G$ be a bounded domain in $\mathbf{R}^{d}$. For each $\zeta \in G$ we assume that there is a maximizer $\left(r_{\zeta}, \rho_{\zeta}\right) \in K$ of

$$
H_{\zeta}(r, \rho)=h(r, \rho)-\phi(r-\zeta)
$$

over $K$ such that $\nabla \phi\left(r_{\zeta}-\zeta\right)=0$. Then,

$$
h_{\phi}(\zeta)=\sup \left\{H_{\zeta}(r, \rho) ;(r, \rho) \in K\right\}
$$

is constant on $G$.

Proof. Clearly, $H_{\zeta}\left(r_{\eta}, \rho_{\eta}\right) \leq h_{\phi}(\zeta)$ for $\eta \in G$. By definition we have

$$
H_{\zeta}\left(r_{\eta}, \rho_{\eta}\right)=H_{\eta}\left(r_{\eta}, \rho_{\eta}\right)+\phi\left(r_{\eta}-\eta\right)-\phi\left(r_{\eta}-\zeta\right),
$$

which yields

$$
h_{\phi}(\eta) \leq h_{\phi}(\zeta)+\phi\left(r_{\eta}-\zeta\right)-\phi\left(r_{\eta}-\eta\right)
$$

Since $\nabla \phi\left(r_{\eta}-\eta\right)=0$, this yields

$$
h_{\phi}(\eta) \leq h_{\phi}(\zeta)+\frac{1}{2} \int_{0}^{1} \nabla^{2} \phi\left(r_{\eta}-\eta-\tau(\zeta-\eta)\right) d \tau(\zeta-\eta) \cdot(\zeta-\eta)
$$

Since $G$ is bounded, it follows that

$$
h_{\phi}(\eta)-h_{\phi}(\zeta) \leq C|\eta-\zeta|^{2}
$$

with $C$ independent of $\eta, \zeta \in G$. Changing the role of $\eta, \zeta$ we end up with

$$
\left|h_{\phi}(\eta)-h_{\phi}(\zeta)\right| \leq C|\eta-\zeta|^{2} \text {. }
$$

This implies that $h_{\phi}$ is differentiable on $G$ whose derivative is always zero. Since $G$ is connected, this means that $h_{\phi}$ is a constant function on $G$.

We shall always use the same $\kappa, \zeta_{0}, p_{0}$ and $\delta$ as in Lemma 7.4. We apply Lemma 7.5 with

$$
\begin{aligned}
& \phi(r)=B_{\epsilon}(r)-p_{0} r, \quad G=\left(\zeta_{0}-\delta, \zeta_{0}+\delta\right), d=1, \\
& h(r, \rho)=w(t, r+y, s, y)-p_{0}\left(r-\zeta_{0}\right)-S(t, s), \\
& \rho=(t, s, y), \quad N=4, \\
& K=\{(r, \rho) ;(t, s) \in[0, T] \times[0, T], \quad r=x-y,(x, y) \in \bar{\Omega} \times \bar{\Omega}\}
\end{aligned}
$$

to get the constancy of $h_{\phi}(\zeta)=g(\zeta)-p_{0}\left(\zeta-\zeta_{0}\right)$. 
7.6. Proposition. The function $\tilde{g}(\zeta)=g(\zeta)-p_{0}\left(\zeta-\zeta_{0}\right)$ is constant on $G=\left(\zeta_{0}-\delta, \zeta_{0}+\delta\right)$.

This gives the information of local behavior of $w$ near a maximizer of $\Phi_{\zeta_{0}}$.

7.7. Proposition. Let $\left(\hat{z}, \hat{z}^{\prime}\right)=(\hat{t}, \hat{z}, \hat{z}, \hat{y})$ be a maximizer of $\Phi_{\zeta_{0}}$, i.e., $\left(\hat{z}, \hat{z}^{\prime}\right) \in \mathcal{A}\left(\zeta_{0}\right)$ with the property that $p_{0}=2\left(\hat{z}-\hat{y}-\zeta_{0}\right) / \epsilon$. Let $u_{0}$ and $v_{0}$ denote

$$
u_{0}(t, x)=u(t, x)-p_{0} x, \quad v_{0}(s, y)=v(s, y)-p_{0}\left(y+\zeta_{0}\right) .
$$

Then $u_{0}(\hat{t}, \cdot)$ and $-v_{0}(\hat{s}, \cdot)$ take their local maximums at $\hat{x}$ and $\hat{y}$ respectively. More precisely

$$
\begin{array}{lll}
u_{0}(\hat{t}, x) \leq u_{0}(\hat{t}, \hat{x}) & \text { for all } \quad x,|x-\hat{x}|<\delta, x \in \Omega, \\
v_{0}(\hat{s}, y) \geq v_{0}(\hat{s}, \hat{y}) & \text { for all } \quad y,|y-\hat{y}|<\delta, y \in \Omega .
\end{array}
$$

Proof Step 1. . We shall prove: for $w_{0}\left(z, z^{\prime}\right)=u_{0}(t, x)-v_{0}(y, s)$ let $E$ denote

$$
E\left(z, z^{\prime}\right)=w_{0}\left(z, z^{\prime}\right)-S(t, s)
$$

where $S$ is the same as in the definition of $\Psi_{\zeta}$. Let $E_{1}$ be

$$
E_{1}=\sup \left\{E\left(z, z^{\prime}\right) ; z=(t, x) \in \bar{Q}, z^{\prime}=(s, y) \in \bar{Q},(x, y) \in \Sigma_{\delta}\right\}
$$

with $\Sigma_{\delta}=\left\{(x, y) \in \bar{\Omega} \times \bar{\Omega} ;\left|x-y-\zeta_{0}-q_{0}\right|<\delta\right\}$ with $q_{0}=\hat{\boldsymbol{x}}-\hat{y}-\zeta_{0}$. Then $E_{1}=\Phi_{\zeta_{0}}\left(\hat{z}, \hat{z}^{\prime}\right)-q_{0}^{2} / \epsilon\left(=E\left(\hat{z}, \hat{z}^{\prime}\right)\right)$.

We first observe that

$$
\Phi_{\zeta}\left(z, z^{\prime}\right)=w_{0}\left(z, z^{\prime}\right)-B_{\varepsilon}(z-y-\zeta)+p_{0}\left(z-y-\zeta_{0}\right)-S(t, s) .
$$

For $\zeta,\left|\zeta-\zeta_{0}\right|<\delta$ recalling that $p_{0}=B_{\epsilon}^{\prime}\left(q_{0}\right)=2\left(x_{\zeta}-y_{\zeta}-\zeta\right) / \epsilon$ (independent of $\zeta)$ with some $\left(t_{\zeta}, x_{\zeta}, s_{\zeta}, y_{\zeta}\right) \in \mathcal{A}(\zeta)$ we rearrange

$$
\begin{aligned}
& \epsilon B_{\epsilon}(x-y-\zeta)-\epsilon p_{0}\left(x-y-\zeta_{0}\right) \\
= & (x-y-\zeta)^{2}-\epsilon p_{0}(x-y-\zeta)-\epsilon p_{0}\left(\zeta-\zeta_{0}\right) \\
= & \left(x-y-\zeta-\left(x_{\zeta}-y_{\zeta}-\zeta\right)\right)^{2} \\
& -\left(x_{\zeta}-y_{\zeta}-\zeta\right)^{2}-2\left(x_{\zeta}-y_{\zeta}-\zeta\right)\left(\zeta-\zeta_{0}\right) \\
= & \left(x-y-\zeta-q_{0}\right)^{2}-q_{0}^{2}-2 q_{0}\left(\zeta-\zeta_{0}\right)
\end{aligned}
$$

to obtain

$$
\Phi_{\zeta}\left(z, z^{\prime}\right)=w_{0}\left(z, z^{\prime}\right)-B_{\epsilon}\left(x-y-\zeta-q_{0}\right)-S(t, s)+q_{0}^{2} / \epsilon+p_{0}\left(\zeta-\zeta_{0}\right) .
$$


Since Proposition 7.6 implies $\tilde{g}(\zeta)=g(\zeta)-p_{0}\left(\zeta-\zeta_{0}\right)$ is constant for $\left|\zeta-\zeta_{0}\right|<$ $\boldsymbol{\delta}$,

$$
\begin{aligned}
\Phi_{\zeta_{0}}\left(\hat{z}, \hat{z}^{\prime}\right) & =\tilde{g}\left(\zeta_{0}\right)=\sup \left\{\tilde{g}(\zeta) ;\left|\zeta-\zeta_{0}\right|<\delta\right\} \\
& =\sup \left\{\Phi_{\zeta}\left(z, z^{\prime}\right)-p_{0}\left(\zeta-\zeta_{0}\right) ;\left(z, z^{\prime}\right) \in \bar{Q} \times \bar{Q},\left|\zeta-\zeta_{0}\right|<\delta\right\} \\
& \geq \sup _{\left|\zeta-\zeta_{0}\right|<\delta} \sup \left\{\Phi_{\zeta}(t, x, s, y)-p_{0}\left(\zeta-\zeta_{0}\right) ;\right. \\
& \left.x-y-q_{0}=\zeta,(t, x, s, y) \in \bar{Q} \times \bar{Q}\right\} .
\end{aligned}
$$

If $x-y-q_{0}=\zeta$, then we see

$$
\begin{aligned}
\Phi_{\zeta}\left(z, z^{\prime}\right)-p_{0}\left(\zeta-\zeta_{0}\right) & =w_{0}\left(z, z^{\prime}\right)-B_{\epsilon}(0)-S(t, s)+q_{0}^{2} / \epsilon \\
& =E\left(z, z^{\prime}\right)+q_{0}^{2} / \epsilon
\end{aligned}
$$

We thus obtain

$$
\Phi_{\zeta_{0}}\left(\hat{z}, \hat{z}^{\prime}\right) \geq E_{1}+q_{0}^{2} / \epsilon .
$$

Since $\hat{x}-\hat{y}-q_{0}=\zeta_{0}$, we see as above that

$$
\Phi_{\zeta_{0}}\left(\hat{z}, \hat{z}^{\prime}\right)=E\left(\hat{z}, \hat{z}^{\prime}\right)+q_{0}^{2} / \epsilon
$$

which proves the last equality in step 1 . Since $(\hat{x}, \hat{y}) \in \Sigma_{\delta}$, the converse inequality

$$
\Phi_{\zeta_{0}}\left(\hat{z}, \hat{z}^{\prime}\right) \leq E_{1}+q_{0}^{2} / \epsilon
$$

holds. The proof of Step 1 is now complete.

Step 2. From Step 1 it follows that

$$
w_{0}(\hat{t}, x, \hat{s}, y)-S(\hat{t}, \hat{s}) \leq w_{0}(\hat{t}, \hat{z}, \hat{s}, \hat{y})-S(\hat{t}, \hat{s})=E\left(\hat{z}, \hat{z}^{\prime}\right)
$$

for $(x, y) \in \Sigma_{\delta}$. Setting $y=\hat{y}$ (resp. $\left.x=\hat{x}\right)$ yields

$$
\begin{array}{lll}
u_{0}(\hat{t}, x) \leq u_{0}(\hat{t}, \hat{x}) & \text { for } \quad|x-\hat{x}|<\delta \text { and } \\
v_{0}(\hat{s}, y) \geq v_{0}(\hat{s}, \hat{y}) & \text { for } \quad|y-\hat{y}|<\delta, & \text { respectively. }
\end{array}
$$

7.8. Remark. If $P$ consists of only zero point, Lemma 7.4 is trivial with $\zeta_{0}=0, \delta=\kappa$ and $p_{0}=0$. In this case $q_{0}=\epsilon p_{0} / 2=0, u_{0}=u$, and $v_{0}=v$ so that proof of Proposition 7.7 is simplified. To understand the main idea of the proof of the Comparison Theorem it is a good idea to consider this special case. 


\subsection{Corollary to Proposition 7.7.}

$$
u_{0}(t, x)-v_{0}(s, y)-S(t, s) \leq u_{0}(\hat{t}, \hat{x})-v_{0}(\hat{s}, \hat{y})-S(\hat{t}, \hat{s})
$$

for all $(x, y) \in \Sigma_{\delta}, t, s \in[0, T]$, where

$$
\begin{aligned}
& \Sigma_{\delta}=\{(x, y) \in \bar{\Omega} \times \bar{\Omega} ;|x-y-(\hat{z}-\hat{y})|<\delta\} \\
& S(t, s)=\gamma /(T-t)+\gamma^{\prime} /(T-s)+(t-s)^{2} / \sigma .
\end{aligned}
$$

This follows immediately from the Step 1 of the proof of Proposition 7.7.

7.10. Proposition on behavioux away from local maximum. Let $\left(\hat{z}, \hat{z}^{\prime}\right)=(\hat{t}, \hat{z}, \hat{z}, \hat{y}), u_{0}$ and $v_{0}$ be the same as in Proposition 7.7. Let $\Omega=$ $(a, b)$. Then, there is $x_{1} \in\left(\hat{x}, b_{1}\right)$ or $y_{1} \in\left(\hat{y}, b_{2}\right)$ such that

$$
u_{0}\left(\hat{t}, x_{1}\right)<u_{0}(\hat{t}, \hat{x}) \text { or } v_{0}\left(\hat{s}, y_{1}\right)>v_{0}(\hat{s}, \hat{y})
$$

with $\eta=\hat{z}-\hat{y}, b_{1}=\min (b, b+\eta), b_{2}=\min (b, b-\eta)$. The same assertion is valid if $\left(\hat{z}, b_{1}\right)$ and $\left(\hat{y}, b_{2}\right)$ are replaced by $\left(a_{1}, \hat{x}\right)$ and $\left(a_{2}, \hat{y}\right)$, respectively, with $a_{1}=\max (a, a+\eta), a_{2}=\max (a, a-\eta)$.

Proof. Here Remark 7.2 is explicitly invoked. We may assume $\hat{\boldsymbol{y}} \geq \hat{\boldsymbol{x}}$. Suppose that the first assertion were false. Then

$$
\begin{array}{ll}
u_{0}(\hat{t}, x) \geq u_{0}(\hat{t}, \hat{x}) & \text { for all } x, \hat{x}<x<b+\eta \text { and } \\
v_{0}(\hat{s}, y) \leq v_{0}(\hat{x}, \hat{y}) & \text { for all } y, \hat{y}<y<b .
\end{array}
$$

Since $u_{0}$ and $-v_{0}$ are uppersemicontinuous on $\bar{Q}$,

$$
u_{0}(\hat{t}, b-\eta) \geq u_{0}(\hat{t}, \hat{z}) \text { and } v_{0}(\hat{s}, b) \leq v_{0}(\hat{s}, \hat{y})
$$

Since $q_{0}=\hat{x}-\hat{y}-\zeta_{0}$ we see

$$
\begin{aligned}
& u_{0}(\hat{t}, b-\eta)-v_{0}(\hat{s}, b)=u(\hat{t}, b-\eta)-p_{0}(b-\eta)-v(\hat{s}, b)+p_{0}\left(b+\zeta_{0}\right) \\
= & u(\hat{t}, b-\eta)-v(\hat{s}, b)+p_{0}\left(\hat{y}-\hat{z}+\zeta_{0}\right) \\
= & u(\hat{t}, b-\eta)-v(\hat{s}, b)-q_{0}^{2} / \epsilon .
\end{aligned}
$$

By Remark 7.2 and Proposition 7.1 (ii) this yields

$$
u_{0}(\hat{t}, \hat{x})-v_{0}(\hat{s}, \hat{y}) \leq u_{0}(\hat{t}, b-\eta)-v_{0}(\hat{s}, b) \leq \xi-q_{0}^{2} / \epsilon=m_{0} / 4-q_{0}^{2} / \epsilon .
$$

Since $\Phi_{\zeta_{0}}(\hat{t}, \hat{z}, \hat{s}, \hat{y}) \geq m_{0}-m_{0} / 8$ by Proposition 7.1 (i), Step 1 of the proof of Proposition 7.7 yields

$$
u_{0}(\hat{t}, \hat{x})-v_{0}(\hat{s}, \hat{y})>E(\hat{t}, \hat{x}, \hat{s}, \hat{y}) \geq 7 m_{0} / 8-q_{0}^{2} / \epsilon
$$


We thus obtain a contradiction:

$$
m_{0} / 4-q_{0}^{2} / \epsilon \geq u_{0}(\hat{t}, \hat{x})-v(\hat{s}, \hat{y})>7 m_{0} / 8-q_{0}^{2} / \epsilon
$$

The proof of the second assertion is the same so is omitted.

D. Preparation for applying the maximum principle.

We shall fix $\kappa$ in Lemma 7.4, say $\kappa=\kappa_{1}$. Key properties of $u_{0}$ and $v_{0}$ are summarized in Proposition 7.7, Corollary 7.9 and Proposition 7.10.

7.11. Applications of sup-convolutions. Since $u_{0}$ and $v_{0}$ in Proposition 7.7 may not be continuous we regularize by taking sup-convolution introduced in $§ 5$. For $\rho \geq 0$ and $\lambda>0$, let $\vartheta(x, \rho, \lambda)$ denote

$$
\vartheta(x, \rho, \lambda)= \begin{cases}(x-\rho)^{2} / \lambda ; & x>\rho \\ 0 ; & |x| \leq \rho \\ (x+\rho)^{2} / \lambda ; & x<-\rho\end{cases}
$$

If $\rho=\lambda(>0)$ we simply write it by $\vartheta(x, \rho)$. We consider sup-convolutions of $u_{0}$ and $-v_{0}$ by $\vartheta$. For $\alpha>0$ let $u_{0}^{\alpha}$ by the sup-convolution of $u_{0}$ in $x$ direction, i.e.,

$$
u_{0}^{\alpha}(t, x)=\left(u_{0}(t, \cdot)\right)^{\alpha}=\sup \left\{u_{0}(t, \eta)-\vartheta(\eta-x, \alpha) ; \eta \in \mathbf{R}\right\},
$$

where we use the convention that $u_{0}=-\infty$ if $\eta$ is outside $\bar{\Omega}$. The infconvolution of $v_{0}$ is defined by $v_{0 \beta}=-\left(-v_{0}\right)^{\beta}$ for $\beta>0$. Both functions $u_{0}^{\alpha}, v_{0 \beta}$ are defined in $[0, T] \times \mathbf{R}$.

7.12. Proposition. Let $(\hat{t}, \hat{x}, \hat{s}, \hat{y}), u_{0}$ and $v_{0}$ be as in Proposition 7.7. Then there is $\alpha_{0}>0$ such that for $0<\alpha \leq \alpha_{0}$

(i) $u_{0}^{\alpha}(\hat{t}, \cdot)$ and $v_{0 \alpha}(\hat{s}, \cdot)$ is faceted at $\hat{x}$ and $\hat{y}$ in $\mathbf{R}$ respectively with slope zero, and $u_{0}^{\alpha}(\hat{t}, \hat{x})=u_{0}(\hat{t}, \hat{x}), v_{0 \alpha}(\hat{z}, \hat{y})=v_{0}(\hat{s}, \hat{y})$,

(ii) the point $\hat{x}$ (resp. $\hat{y}$ ) belongs to the interior of the faceted region $R\left(u_{0}^{\alpha}(\hat{t}, \cdot), \hat{z}\right)\left(\right.$ resp. $\left.R\left(v_{0 \alpha}(\hat{s}, \cdot), \hat{y}\right)\right)$,

(iii)

$$
\begin{aligned}
& \hat{y} \in\left\{R\left(u_{0}^{\alpha}(\hat{t}, \cdot), \hat{z}\right)-\eta\right\} \cap R\left(v_{0 \alpha}(\hat{s}, \cdot), \hat{y}\right) \subset \Omega \text { and } \\
& \hat{\boldsymbol{x}} \in R\left(u_{0}^{\alpha}(\hat{t}, \cdot), x\right) \cap\left\{R\left(v_{0 \alpha}(\hat{\boldsymbol{s}}, \cdot), \hat{y}\right)+\eta\right\} \subset \Omega \quad \text { with } \quad \eta=\hat{x}-\hat{y} .
\end{aligned}
$$

Proof. By Lemma 5.1 convolution $u_{0}^{\alpha}$ is locally Lipschitz continuous in the space variable. Since $u_{0}(\hat{t}, \cdot)$ takes its local maximum at $\hat{x}$, by Theorem 5.3 on convolution with faceted functions $u_{0}^{\alpha}(\hat{t}, \cdot)$ is faceted at $\boldsymbol{x}$ in $\mathbf{R}$ for small $\alpha$ and that $u_{0}^{\alpha}(\hat{t}, \cdot)$ is constant $u_{0}(\hat{t}, \hat{x})$ around $\hat{x}$ from a fundamental property of our sup-convolutions; note that $u_{0}$ and $-v_{0}$ are bounded from above. This 
proves (i) and (ii) for $u_{0}^{\alpha}$. The proof for $v_{0 \alpha}$ is the same by taking $\alpha_{0}$ smaller if necessary.

It remains to prove (iii). Here we use Proposition 7.10. We again recall a fundamental property for sup-convolution; if there is $b_{1}>x_{1}>\hat{x}$ such that $u_{0}\left(\hat{t}, x_{1}\right)<u_{0}(\hat{t}, \hat{x})$, then

$$
\sup R\left(u_{0}^{\alpha}(\hat{t}, \cdot), \hat{x}\right)<b_{1}
$$

for sufficiently small $\alpha$ (provided that $u_{0}(\hat{t}, \cdot)$ is uppersemicontinuous which is fulfilled in our setting). Thus Proposition 7.10 implies the desired inclusion by taking smaller $\alpha_{0}$ if necessary.

In Corollary 7.9 we have

$$
u_{0}(t, x)-v_{0}(s, y)-S(t, s) \leq u_{0}(\hat{t}, \hat{x})-v_{0}(\hat{s}, \hat{y})-S(\hat{t}, \hat{z})
$$

for all $(x, y) \in \Sigma_{\delta}, t, s \in[0, T]$. We shall derive the similar inequality for $u_{0}^{\alpha}$ and $v_{0 \alpha}$. We introduce a barrier for $|x-y-(\hat{x}-\hat{y})|>\delta$ so that the region where the inequality is valid contains all $x, y \in \mathbf{R}$.

7.13. Proposition. Let $\vartheta$ be as in $\$ 7.11$. Let $(\hat{t}, \hat{x}, \hat{z}, \hat{y}), u_{0}$ and $v_{0}$ be as in Proposition 7.7.

(i) $u_{0}(t, x)-v_{0}(s, y)-S(t, s)-\vartheta(x-y-\eta, \lambda) \leq u_{0}(\hat{t}, \hat{x})-v_{0}(\hat{s}, \hat{y})-S(\hat{t}, \hat{s})$

for all $(t, s),(s, y) \in \bar{Q} \times \bar{Q}$ provided that $\lambda>0$ is sufficiently small, i.e. $\lambda \leq \lambda_{0}$ for some $\lambda_{0}>0$, where $\eta=\hat{z}-\hat{y}$.

(ii) Let $\alpha_{0}$ be as in Proposition 7.12. Then

$$
\begin{aligned}
u_{0}^{\alpha}(t, x)-v_{0 \alpha}(s, y) \leq & u_{0}^{\alpha}(\hat{t}, \hat{x})-v_{0 \alpha}(\hat{s}, \hat{y})+\vartheta\left(x-y-\eta, \lambda_{0} / 2\right) \\
& +S(t, s)-S(\hat{t}, \hat{s})
\end{aligned}
$$

for all $(t, x),(s, y) \in[0, T] \times \mathbf{R}$ provided that $0<\alpha \leq \alpha_{1}=\min \left(\alpha_{0}, \lambda_{0} / 4\right)$.

Proof. (i) Let $E_{1}$ and $E_{2}$ be

$$
\begin{aligned}
& E_{1}=\sup \left\{E(t, x, s, y) ;(t, x) \in \bar{Q}, \quad(s, y) \in \bar{Q}, \quad(x, y) \in \Sigma_{\delta}\right\} \\
& E_{2}=\sup \left\{E(t, x, s, y) ;(t, x) \in \bar{Q}, \quad(s, y) \in \bar{Q}, \quad(x, y) \notin \Sigma_{\delta}\right\}
\end{aligned}
$$

with $E=u_{0}(t, x)-v_{0}(s, y)-S(t, s)$ and $\Sigma_{\delta}=\{(x, y) \in \bar{\Omega} \times \bar{\Omega} ;|x-y-(\hat{x}-\hat{y})|<$ $\delta$ \}. Step 1 of Proposition 7.7 yields $E_{1}=u_{0}(\hat{t}, \hat{x})-v_{0}(\hat{s}, \hat{y})-S(\hat{t}, \hat{z})$. If $E_{2} \leq E_{1}$, the inequality in (i) is trivial since $\vartheta \geq 0$. If $E_{2}>E_{1}$, we take $\lambda_{0}$ satisfying

$$
\vartheta\left(\delta, \lambda_{0}\right)=E_{2}-E_{1}
$$


Since $\vartheta\left(x-y-\eta, \lambda_{0}\right) \geq \vartheta\left(\delta, \lambda_{0}\right)$ for $(x, y) \notin \Sigma_{\delta}$ and since $\vartheta$ is monotone in $\lambda$, the proof of (i) is complete.

(ii) From (i) it follows that

$$
\begin{aligned}
u_{0}(t, \tilde{x}) & -\vartheta(x-\tilde{x}, \alpha)-\left(v_{0}(s, \tilde{y})+\vartheta(y-\tilde{y}, \alpha)\right) \\
\leq & u_{0}(\hat{t}, \hat{x})-v_{0}(\hat{s}, \hat{y}) \\
& +\vartheta\left(\tilde{x}-\tilde{y}-\eta, \lambda_{0}\right)-\vartheta(x-\tilde{x}, \alpha)-\vartheta(y-\tilde{y}, \alpha) \\
& +S(t, s)-S(\hat{t}, \hat{s}) .
\end{aligned}
$$

Since $u_{0}=-v_{0}=+\infty$ outside $\Omega$, taking the supremum of both sides for $\tilde{\boldsymbol{x}}, \tilde{y} \in \mathbf{R}$ we get

$$
\begin{aligned}
u_{0}^{\alpha}(t, x) & -v_{0 \alpha}(s, y) \leq u_{0}(\hat{t}, \hat{x})-v_{0}(\hat{s}, \hat{y}) \\
& +\vartheta\left(x-y-\eta, \lambda_{0}-2 \alpha\right)+S(t, s)-S(\hat{t}, \hat{s})
\end{aligned}
$$

for all $(t, x),(s, y) \in[0, T] \times \mathbf{R}$. Here we have invoked the composition rule for sup-convolution (Lemma 5.5 (ii)):

$$
\begin{aligned}
& \sup \left\{\vartheta\left(x-y-\eta, \lambda_{0}\right)-\vartheta(x-\tilde{x}, \alpha)-\vartheta(y-\tilde{y}, \alpha) ; \tilde{x}, \tilde{y} \in \mathbf{R}\right\} \\
& \quad=\vartheta\left(z-y-\eta, \lambda_{0}-2 \alpha\right)
\end{aligned}
$$

Since $u_{0}^{\alpha}(\hat{t}, \hat{x})=u_{0}(\hat{t}, \hat{x})$ and $v_{0 \alpha}(\hat{s}, \hat{y})=v_{0}(\hat{s}, \hat{y})$ by Proposition 7.12 (i) and since $\lambda_{0}-2 \alpha \leq \lambda_{0} / 2$ for $\alpha \leq \alpha_{1}$, the proof of (ii) is now complete.

E. Application of the maximum principle.

The function $u_{0}^{\alpha}+p_{0} \approx$ is essentially an admissible super function (of infinitesimal version defined in $\S 6.5)$ at $(\hat{t}, \hat{x}) \in Q$ except that the faceted region $R\left(u_{0}^{\alpha}(\hat{t}, \cdot), \hat{x}\right)$ may contain the boundary point of $\Omega$. We apply the Maximum Principle of time direction 4.5 and its Corollary 4.6 to Proposition 7.13 to get useful admissible super functions.

Here we use the notation $\tilde{N}^{-1}$ of semineighborhood defined in $\$ 6.3$.

7.14. Proposition. Let $(\hat{t}, \hat{x}, \hat{s}, \hat{y}), u_{0}$ and $v_{0}$ be as in Proposition 7.7. There are a (real-valued) uppersemicontinuous function $\bar{u}$ and a lowersemicontinuous function $\underline{v}$ defined in $Q$ such that

(i) $\bar{u}(t, \cdot) \in C(\Omega), \underline{v}(s, \cdot) \in C(\Omega)$ for each $t, s \in(0, T) ; \bar{u}(\hat{t}, \cdot)$ and $\underline{v}(\hat{s}, \cdot)$ are faceted at $\hat{x}$ and $\hat{y}$, respectively, with slope zero in $\Omega ; u_{0}^{\alpha} \leq \bar{u}$ and $v_{0 \alpha} \geq \underline{v}$ in $Q ; \bar{u}(\hat{t}, \hat{z})=u_{0}^{\alpha}(\hat{t}, \hat{x})$ and $\underline{v}(\hat{s}, \hat{y})=v_{0 \alpha}(\hat{s}, \hat{y})$.

(ii) $R(\bar{u}(\hat{t}, \cdot), \hat{x})=R\left(u_{0}^{\alpha}(\hat{t}, \cdot), \hat{x}\right) \cap\left\{R\left(v_{0 \alpha}(\hat{s}, \cdot), \hat{y}\right)+\eta\right\}$, $R(\underline{v}(\hat{s}, \cdot), \hat{y})=\left\{R\left(u_{0}^{\alpha}(\hat{t}, \cdot), \hat{z}\right)-\eta\right\} \cap R\left(v_{0 \alpha}(\hat{s}, \cdot), \hat{y}\right)$,

so that $L(\bar{u}(\hat{t}, \cdot), \hat{z})=L(\underline{v}(\hat{s}, \cdot), \hat{y})$, where $\eta=\hat{x}-\hat{y}$.

(iii) $\chi(\bar{u}(\hat{t}, \cdot), \hat{z})+\chi(-\underline{v}(\hat{s}, \cdot), \hat{y}) \leq 0$. 
(iv)For some $\tilde{N}^{-1}(\bar{u}(\hat{t}, \cdot), \hat{x})$ the inequality

$$
\bar{u}(t, x)-\bar{u}(\hat{t}, \hat{z}) \leq S(t, \hat{s})-S(\hat{t}, \hat{s})
$$

holds for all $(t, x) \in(0, T) \times \tilde{N}^{-1}(\bar{u}(\hat{t}, \cdot), \hat{x}) ;$ for some $\tilde{N}^{-1}((-\bar{v})(\hat{s}, \cdot), \hat{y})$ the inequality

$$
\underline{v}(s, y)-\underline{v}(\hat{s}, \hat{y}) \geq S(\hat{t}, \hat{s})-S(\hat{t}, s)
$$

holds for all $(s, y) \in(0, T) \times \tilde{N}^{-1}(-\underline{v}(\hat{s}, \cdot), \hat{y})$. Here $\alpha$ is chosen so that $\alpha \leq \alpha_{1}$, where $\alpha_{1}$ is as in Proposition 7.13.

Proof. We apply Corollary 4.6 of the maximum principle to Propositions 7.12 and 7.13 so that we find desired $\bar{u}$ and $\underline{v}$. Here $u_{0}^{\alpha}$, and $-v_{0 \alpha}$, correspond to $u_{1}$, and $u_{2}$, of Corollary 4.6, respectively. Note that Proposition 7.12 (iii) plays an important role to apply Corollary 4.6.

7.15. Proposition. Let $U$ and $V$ denote

$U(t, x)=\bar{u}(t, x)+p_{0} x, \quad V(s, y)=\underline{v}(s, y)+p_{0}\left(y+\zeta_{0}\right) \quad$ for $(t, x),(s, y) \in Q$

Then $U$ is an admissible super function at $(\hat{t}, \hat{x})$ in $Q$ and $V$ is an admissible sub function at $(\hat{t}, \hat{x})$ in $Q$. Moreover, $U(\hat{t}, \cdot)$ is faceted at $\hat{x} \in$ int $R(U(\hat{t}, \cdot), \hat{x})$ and $\mathcal{T}_{P}^{+} U(\hat{t}, \hat{x}) \ni S_{t}(\hat{t}, \hat{s}) ; V(\hat{s}, \cdot)$ is faceted at $\hat{y} \in$ int $R(V(\hat{s}, \cdot), \hat{y})$ and $\mathcal{T}_{P}^{-} V(\hat{\mathrm{s}}, \hat{y}) \ni-S_{0}(\hat{t}, \hat{\mathrm{s}})$, where $\mathcal{T}_{P}^{+}$and $\mathcal{T}_{P}^{-}$are as in $\S 6.4$.

Proof. We shall only prove that $U$ is an admissible super function since the statement for $V$ can be proved similarly. By Proposition 7.14 (i) $U$ is uppersemicontinuous in $Q$ and $U(t, \cdot) \in C(\Omega)$ for each $t \in(0, T)$. Moreover $U(\hat{t}, \cdot)$ is $P$-faceted at $\hat{\boldsymbol{x}}$ in $\Omega$ with slope $p_{0}$. Since $\hat{\boldsymbol{z}}$ (resp. $\hat{\boldsymbol{y}}$ ) is an interior point of $R\left(u_{0}^{\alpha}(\hat{t}, \cdot), \hat{z}\right)$ (resp. $R\left(v_{0 \alpha}(\hat{s}, \cdot), \hat{y}\right)$ ) by Proposition 7.12 (ii), we see, by Proposition 7.14 (ii), $\hat{x}$ (resp. $\hat{y}$ ) is an interior point of $R(U(\hat{t}, \cdot), \hat{x})$ (resp. $R(V(\hat{s}, \cdot), \hat{y}))$.

It remains to prove that $\mathcal{T}_{P}^{+} U(\hat{t}, \hat{x})$ contains $S_{t}(\hat{t}, \hat{z})$ as an element. Since

$$
S(t, \hat{s})-S(\hat{t}, \hat{s}) \leq S_{t}(\hat{t}, \hat{s})(t-\hat{t})+\omega(|t-\hat{t}|)|t-\hat{t}|
$$

for all $t \in \mathbf{R}$ with some modulus $\omega$, Proposition 7.14 (iv) yields

$$
U(t, x)-U(\hat{t}, \hat{x}) \leq p_{0}(x-\hat{x})+S_{t}(\hat{t}, \hat{s})(t-\hat{t})+\omega(|t-\hat{t}|)|t-\hat{t}|
$$

for $(t, x) \in(0, T) \times \tilde{N}^{-1}(U(\hat{t}, \cdot), \hat{x})$. This implies that $S_{t}(\hat{t}, \hat{z}) \in \mathcal{T}_{P}^{+} U(\hat{t}, \hat{z})$.

F. Completion of the proof for Case $I$.

By definition of sub- and supersolutions $u^{*}$ and $-v_{*}$ are uppersemicontinuous in $[0, T) \times \bar{\Omega}$ with values in $\mathbf{R} \cup\{-\infty\}$. To show our Comparison Theorem 
it suffices to prove that the restriction of $u^{*}$ and $v_{*}$ on $\left[0, T^{\prime}\right) \times \bar{\Omega}$ (denoted by $u^{\prime}$ and $v^{\prime}$ respectively) fulfills $u^{\prime} \leq v^{\prime}$ on $\left[0, T^{\prime}\right) \times \bar{\Omega}$ for each $T^{\prime}>0$. Clearly $\left(u^{\prime}\right)^{*}-\left(v^{\prime}\right)_{*} \leq u^{*}-v_{*}$ on $\left[0, T^{\nu}\right] \times \bar{\Omega}$, so that $u^{*} \leq v_{*}$ on $\partial_{p} Q$ implies $\left(u^{\prime}\right)^{*} \leq\left(v^{\prime}\right)_{*}$ on

$$
\bar{\partial}_{p} Q^{\prime}=\overline{\partial_{p} Q^{\prime}}=\left[0, T^{\prime}\right] \times \partial \Omega \cup\{0\} \times \bar{\Omega} .
$$

By writing $T^{\prime}$ by $T, u^{\prime}$ by $u$, and $v^{\prime}$ by $v$ we may assume $u \leq v$ on $\bar{\partial}_{p} Q$ and that $u$ and $-v$ are uppersemicontinuous in $\bar{Q}$ with values $\mathbf{R} \cup\{-\infty\}$. If $F$ depends on $t$, by writing $T^{\prime}$ by $T$ we may assume

(F3') $F$ is uniformly continuous in $[0, T] \times[-K, K] \times \mathbf{R}$ for each $K>0$.

Suppose that the conclusion of the Theorem were false. We may assume that $m_{0}$ satisfies the assumption of Proposition 7.1. We fix $\epsilon_{0}, \sigma_{0}, \gamma_{0}, \gamma_{0}^{\prime}$ as in Remark 7.2 and assume $0<\epsilon<\epsilon_{0}, 0<\sigma<\sigma_{0}, 0<\gamma<\gamma_{0}, 0<\gamma^{\prime}<\gamma_{0}^{\prime}$. Since $\bar{Q}$ is compact and $u$ and $-v$ are uppersemicontinuous there is always a maximizer $\left(t_{\zeta}, x_{\zeta}, s_{\zeta}, y_{\zeta}\right)$ of $\Phi_{\zeta}$ over $\bar{Q} \times \bar{Q}$ in Proposition 7.1.

If we assume Case I, we end up with Propositions 7.12-15. Let $p_{0}$ and $\zeta_{0}$ be as in Lemma 7.4. Let $(\hat{t}, \hat{z}, \hat{s}, \hat{y})$ be a maximizer of $\Phi_{\zeta_{0}}$ with $p_{0}=$ $2\left(\hat{z}-\hat{y}-\zeta_{0}\right) / \epsilon$. Let $U$ be as in Proposition 7.15. Then

$$
\max _{Q}(u-U)=\max _{Q}\left(u_{0}-\bar{u}\right) \leq \max _{Q}\left(u_{0}-u_{0}^{\alpha}\right)
$$

since $u_{0}^{\alpha} \leq \bar{u}$ by Proposition 7.14 (i). Note that $u_{0}^{\alpha}(\hat{t}, \hat{x})=u_{0}(\hat{t}, \hat{x})$ by Proposition 7.12 (i) and that $u_{0} \leq u_{0}^{\alpha}$ by the definition of sup-convolution. We thus observe

$$
\max _{Q}(u-U)=0
$$

Similarly, one can prove

$$
\min _{Q}(v-V)=0 \text {. }
$$

Since $U$ is an admissible super function at $(\hat{t}, \hat{x})$ in $Q$ and since $u$ is a subsolution we have, by the definition of infinitesimal version 6.6 , and Theorems 6.8 and 6.9 ,

$$
S_{t}(\hat{t}, \hat{s})+F\left(\hat{t}, p_{0}, \Delta \chi_{U} / L_{U}\right) \leq 0
$$

with $\chi_{U}=\chi(U(\hat{t}, \cdot), \hat{x}), L_{U}=L(U(\hat{t}, \cdot), \hat{x})$ and $\Delta=W^{\prime}\left(p_{0}+0\right)-W^{\prime}\left(p_{0}-0\right)$. Similarly,

$$
-S_{\imath}(\hat{t}, \hat{s})+F\left(\hat{s}, p_{0}, \Delta \chi_{V} / L_{V}\right) \geq 0 .
$$

with $\chi_{V}=\chi(V(\hat{s}, \cdot), \hat{y}), L_{V}=L(V(\hat{s}, \cdot), \hat{y})$. Subtracting the second inequality from the first yields

$$
\gamma /(T-\hat{t})^{2}+\gamma^{\prime} /(T-\hat{s})^{2}+F\left(\hat{t}, p_{0}, \Delta \chi_{U} / L_{U}\right)-F\left(\hat{s}, p_{0}, \Delta \chi_{V} / L_{V}\right) \leq 0
$$


By Proposition 7.14 (ii), (iii) we see

$$
\begin{aligned}
& \chi_{U}=\chi(\bar{u}(\hat{t}, \cdot), \hat{x}) \leq-\chi(-\underline{v}(\hat{s}, \cdot), \hat{y})=\chi(\underline{v}(\hat{s}, \cdot), \hat{y})=\chi_{V} \quad \text { and } \\
& L_{U}=L(\bar{u}(\hat{t}, \cdot), \hat{x})=L(\underline{v}(\hat{s}, \cdot), \hat{y})=L_{V}
\end{aligned}
$$

which yields

$$
\chi_{U} / L_{U} \leq \chi_{V} / L_{V}
$$

By the monotonicity (F2) of $F$ we now obtain

$$
\frac{\gamma}{(T-\hat{t})^{2}}+\frac{\gamma^{\prime}}{(T-\hat{s})^{2}}+F\left(\hat{t}, p_{0}, \Delta \frac{\chi_{U}}{L_{U}}\right)-F\left(\hat{s}, p_{0}, \Delta \frac{\chi_{U}}{L_{U}}\right) \leq 0
$$

This yields

$$
\left(\gamma+\gamma^{\prime}\right) / T^{2} \leq \omega_{1}(|\hat{t}-\hat{s}| ; \epsilon),
$$

where $\omega_{1}(t ; \epsilon)$ is a modulus of continuity of $F(t, p, X)$ for $|p| \leq K$ and $X \in \mathbf{R}$ with $K \equiv 2 M^{1 / 2} / \epsilon^{1 / 2}$, provided that $\left|p_{0}\right| \leq K$; the existence of such a modulus is guaranteed by (F3'). Indeed since Proposition 7.1 (ii) implies

$\left|B_{\epsilon}^{\prime}\left(x_{\zeta}-y_{\zeta}-\zeta\right)\right|=\left|2\left(x_{\zeta}-y_{\zeta}-\zeta\right) / \epsilon\right| \leq 2 M^{1 / 2} / \epsilon^{1 / 2}=K \quad$ for $\zeta,|\zeta| \leq \kappa_{0}(\epsilon)$, we have $\left|p_{0}\right| \leq K$; note that $K$ is independent of parameters $\sigma, \gamma, \gamma^{\prime}$ other than $\epsilon$ so is $\omega_{1}$. By Proposition 7.1 (ii) we have

$$
\left(\gamma+\gamma^{\prime}\right) / T^{2} \leq \omega_{1}(|\hat{t}-\hat{s}| ; \epsilon) \leq \omega_{1}\left((M \sigma)^{1 / 2} ; \epsilon\right)
$$

for all $\epsilon, \sigma, \gamma, \gamma^{\prime}$ with $0<\epsilon<\epsilon_{0}, 0<\sigma<\sigma_{0}, 0<\gamma<\gamma_{0}, 0<\gamma^{\prime}<\gamma_{0}^{\prime}$ provided that Case I holds. As shown in Case II similar inequality (7.3) holds for Case II with modulus $\omega_{2}(\cdot, \epsilon)$ independent of $\sigma, \gamma, \gamma^{\prime}$. In both cases if $\sigma$ is taken small so that

$$
\omega_{i}\left((M \sigma)^{1 / 2}\right)<\left(\gamma+\gamma^{\prime}\right) / T^{2}, \quad i=1,2
$$

then we get a contradiction. If $F$ is independent of $t,(7.1)$ immediately yields a contradiction: $\left(\gamma+\gamma^{\prime}\right) / T^{2}<0$ without using $\left(\mathrm{F3}^{\prime}\right)$.

\section{G. Case II.}

We use the same choice of parameters $\epsilon, \gamma, \gamma^{\prime}$ and $\sigma$ as in $\S F$. The proof for Case II is standard so we outline it. By the assumption of Case II there is $\zeta_{j} \rightarrow 0$ such that $\hat{\Psi}_{x}=2\left(x_{j}-y_{j}-\zeta_{j}\right) / \epsilon$ does not belong to $P$ for some maximizer $\left(t_{j}, x_{j}, s_{j}, y_{j}\right)$ of $\Phi_{\zeta_{j}}$. We apply the maximum principle for semicontinuous functions [CIL] and observe that for each $\mu>0$ there are $2 \times 2$ symmetric matrices $X$ and $Y$ such that

$$
\begin{aligned}
& \left(\hat{\Psi}_{t}, \hat{\Psi}_{x}, X\right) \in \bar{J}_{Q}^{2,+} u\left(t_{j}, x_{j}\right) \\
& \left(\hat{\Psi}_{\iota}, \hat{\Psi}_{y},-Y\right) \in \bar{J}_{Q}^{2,+}(-v)\left(s_{j}, y_{j}\right) \\
& -\left(\frac{1}{\mu}+|A|\right) I \leq\left(\begin{array}{ll}
X & 0 \\
0 & -Y
\end{array}\right) \leq A+\mu A^{2}
\end{aligned}
$$


with $A=D^{2} \Psi_{\zeta_{j}}\left(t_{j}, x_{j}, s_{j}, y_{j}\right)$ i.e., the Hessian of $\Psi_{\zeta_{j}}$, where $|A|$ is the operator norm of $A$ as a self adjoint operator and $I$ is $4 \times 4$ matrix; $\hat{\Psi}_{t}$ and $\hat{\Psi}_{y}$ are the derivative of $\Psi_{\zeta_{j}}$ with respect to $t$ and $y$ at $\left(t_{j}, x_{j}, s_{j}, y_{j}\right)$, respectively. Here $J_{Q}^{2,+}$ denotes the set of second order super jets and $\bar{J}_{Q}^{2,+}$ is the 'closure' in the sense of semijets; see [CIL] for definitions. It is not difficult [OKS] to derive from first two inclusion that

$$
\begin{aligned}
& \left(\hat{\Psi}_{t}, \hat{\Psi}_{\varpi}, X_{22}\right) \in \overline{\mathcal{P}}_{Q}^{2,+} u\left(t_{j}, z_{j}\right), \\
& \left(-\hat{\Psi}_{\bullet},-\hat{\Psi}_{y}, Y_{22}\right) \in \overline{\mathcal{P}}_{Q}^{2,-} v\left(s_{j}, y_{j}\right)
\end{aligned}
$$

where $\overline{\mathcal{P}}_{Q}^{2, \pm}$ denotes the parabolic version of $\bar{J}_{Q}^{2, \pm} ; \overline{\mathcal{P}}_{Q}^{2, \pm}$ is defined in $\S 6.6$. Here $X_{22}$ (resp. $Y_{22}$ ) denotes the $(2,2)$ component of $X$ (resp. $Y$ ). By a standard argument our matrix inequality yields $X_{22} \leq Y_{22}$.

Since $\hat{\Psi}_{z}=-\hat{\Psi}_{y}$ does not belong to $P$ and since $u$ and $v$ are sub- and supersolution, (by Definition 6.6 and Theorems 6.8 and 6.9) we have

$$
\begin{aligned}
& \hat{\Psi}_{t}+F\left(t_{j}, \hat{\Psi}_{z}, W^{\prime \prime}\left(\hat{\Psi}_{x}\right) X_{22}\right) \leq 0, \\
& -\hat{\Psi}_{s}+F\left(s_{j},-\hat{\Psi}_{y}, W^{\prime \prime}\left(-\hat{\Psi}_{y}\right) Y_{22}\right) \geq 0,
\end{aligned}
$$

where $X_{22}$ (resp. $Y_{22}$ ) is the $(2,2)$ component of $X$ (resp. $Y$ ). Subtracting the second from the first yields

$$
\begin{aligned}
\frac{\gamma}{\left(T-t_{j}\right)^{2}}+\frac{\gamma^{\prime}}{\left(T-s_{j}\right)^{2}} & +F\left(t_{j}, \hat{\Psi}_{x}, W^{\prime \prime}\left(\hat{\Psi}_{z}\right) X_{22}\right) \\
& -F\left(s_{j}, \hat{\Psi}_{\infty}, W^{\prime \prime}\left(\hat{\Psi}_{x}\right) X_{22}\right) \leq 0
\end{aligned}
$$

if we use $X_{22} \leq Y_{22},(\mathrm{~F} 2)$ and $\hat{\Psi}_{\imath}=-\hat{\Psi}_{y}$. Since Proposition 7.1 (ii) yields that $\left|\hat{\Psi}_{a}\right|$ is bounded by $K$ in $\S \mathrm{F}$, as in $\S \mathrm{F}$ this inequality yields

$$
\left(\gamma+\gamma^{\prime}\right) / T^{2} \leq \omega_{2}\left(\left|t_{j}-s_{j}\right| ; \epsilon\right) \leq \omega_{2}\left((M \sigma)^{1 / 2} ; \epsilon\right)
$$

with some modulus $\omega_{2}$ for all $\epsilon, \sigma, \gamma, \gamma^{\prime}$ with $0<\epsilon<\epsilon_{0}, 0<\sigma<\sigma_{0}, 0<$ $\gamma<\gamma_{0}, 0<\gamma^{\prime}<\gamma_{0}^{\prime}$, provided that Case II holds. This is what we would like to prove. (We did not send $\zeta_{j} \rightarrow 0$; the existence of one $\zeta_{j}$ is enough to get a contradiction.) Note that (7.2) immediately yields a contradiction $\left(\gamma+\gamma^{\prime}\right) / T^{2}<0$ if $F$ is independent of $t$; we do not invoke (F3'). We have thus proved our Comparison Theorem.

H. Periodic case, proof of Theorem 3.2.

The method of the proof is essentially the same. Since $u$ and $v$ are $\varpi$ periodic in space, we observe that any maximizer $\left(t_{6}, x_{6}, s_{6}, y_{6}\right)$ of $\Phi_{6}$ satisfies 
$\left|x_{\zeta}-y_{\zeta}\right| \leq \varpi$; we may always assume its existence and that $0 \leq x_{\zeta} \leq \varpi_{,} \leq \leq$ $y_{\zeta} \leq \varpi$. The conclusion of Proposition 7.1 and Remark 7.2 is still valid for such maximizers where $\Omega$ is replaced by $\mathbf{R}$. To define $\mathcal{A}(\zeta)$ we replace $\Omega$ by R. Although graph $\mathcal{A}$ is only closed, graph $\mathcal{B}$ is still compact as claimed in Proposition 7.3 since $0 \leq x_{\zeta}, y_{\zeta} \leq \varpi$. The argument in $\$ 7.4 \$ 7.9$ is still valid if $\Omega$ is replaced by $R$. Propositions 7.10 and 7.12 (iii) should be altered because there is no boundary of $\mathbf{R}$. If $P$ does not contain zero so that $p_{0} \neq 0$, then the faceted regions $R\left(u_{0}^{\alpha}(\hat{t}, \cdot), \hat{x}\right)$ and $R\left(v_{0 \alpha}(\hat{s}, \cdot), \hat{y}\right)$ has the length less than $\varpi$ since $u_{0}^{\alpha}+p_{0} x$ and $v_{0 \alpha}+p_{0}\left(y+\zeta_{0}\right)$ is periodic in the space variable. We apply the maximum principle as in Proposition 7.14. Actually, we have Proposition 7.14 with $\Omega$ replaced by

$$
\Omega^{\prime}=(\hat{x}-\varpi, \hat{z}+\varpi) \cup(\hat{y}-\varpi, \hat{y}+\varpi)
$$

Since Proposition 7.13 does not apply to our setting with $\Omega=\mathbf{R}$, the rest of the proof is the same as $\$ \mathrm{E}-\S \mathrm{G}$.

It remains to discuss the case that $P$ contains zero. If $p_{0} \neq 0$ in Lemma 7.4 , then we argue in the same way. If $p_{0}=0$, then either $L\left(u_{0}^{\alpha}(\hat{t}, \cdot), \hat{x}\right)<\varpi$ or $R\left(u_{0}^{\alpha}(\hat{t}, \cdot), \hat{x}\right)=\mathbf{R}$ and the same for $v_{0 \alpha}(\hat{s}, \cdot)$. Unless both

$$
R\left(u_{0}^{\alpha}(\hat{t}, \cdot), \hat{x}\right)=\mathbf{R} \quad \text { and } \quad R\left(v_{0_{\alpha}}(\hat{s}, \cdot), \hat{y}\right)=\mathbf{R}
$$

hold, Proposition 7.14 still applies with $\Omega$ replaced by $\Omega^{\prime}$ by $\Omega^{\prime}$. We thus assume that both $u_{0}^{\alpha}(\hat{t}, \cdot)$ and $v_{0 \alpha}(\hat{s}, \cdot)$ are constant functions. For each large $\ell$ we consider a nonnegative continuous function $f$ such that $f(x)=0$ if and only if $|x| \leq \ell / 2$. We set $\bar{u}(t, x)=u_{0}^{\alpha}(t, x)+f(x-\hat{x}), \underline{v}(s, y)=v_{0 \alpha}(s, y)-$ $f(y-\hat{y})$ and observe that properties (i)(iv) of Proposition 7.14 (with $\Omega=\mathbf{R}$ ) hold; note that since $\chi(\bar{u}(\hat{t}, \cdot), \hat{x})>0$ and $\chi(-\underline{v}(\hat{s}, \cdot), \hat{y})>0$ so (iii) is violated but $\tilde{N}^{-1}(\bar{u}(\hat{t}, \cdot), \hat{x})=R(\bar{u}(\hat{t}, \cdot), \hat{x})$ and $\tilde{N}^{-1}(-\underline{v}(\hat{s}, \cdot), \hat{y})=R(\underline{v}(\hat{s}, \cdot), \hat{y})$ so (iv) is trivially follows from Proposition 7.13. We also note that $L(\bar{u}(\hat{t}, \cdot), \hat{x})=$ $L(\underline{v}(\hat{s}, \cdot), \hat{y})=\ell$. We may apply Proposition 7.15 with $U=\bar{u}$ and $V=\underline{v}$. As in $\S \mathbf{F}$ we end up with

$$
\gamma /(T-\hat{t})^{2}+\gamma^{\prime} /(T-\hat{s})^{2}+F\left(\hat{t}, 0, \Delta \chi_{U} / L_{U}\right)-F\left(\hat{s}, 0, \Delta \chi_{V} / L_{V}\right) \leq 0
$$

which yields

$$
\left(\gamma+\gamma^{\prime}\right) / T^{2} \leq \omega_{3}(|2 \Delta / \ell|+|\hat{t}-\hat{s}|) \text { for large } \ell
$$

since $L_{U}=L_{V}=\ell$. Here $\omega_{3}$ is a modulus of continuity of $F(t, 0, X)$ on $[0, T] \times[-1,1]$; here we do not invoke (F3'). Sending $\ell \rightarrow \infty$ yields

$$
\left(\gamma+\gamma^{\prime}\right) / T^{2} \leq \omega_{3}(|\hat{t}-\hat{s}|)
$$


which again leads a contradiction as in $\S F$.

Our argument shows at least formally that the weighted curvature

$$
\Lambda_{W}\left(u_{0}^{\alpha}(\hat{t}, \cdot), \hat{x}\right)=0 \quad \text { if } \quad L\left(u_{0}^{\alpha}(\hat{t}, \cdot), \hat{x}\right)=\infty .
$$

\section{§8. Perron type Existence Theorem.}

We shall give the proof of Perron type Existence Theorem 3.3 and Theorem 3.4 for periodic functions. Let $\Omega$ be an open (possibly umbounded) interval and $Q=(0, T) \times \Omega$.

8.1. Lemma. Assume conditions (F1) and (F2). Let $S$ be a nonempty family of a subsolution of $(E)$. Let $u$ be a function defined on $Q$ by

$$
u(t, x)=\sup \{v(t, x) ; v \in S\} \quad \text { for }(t, x) \in Q .
$$

Suppose $u^{*}<\infty$ in $[0, T) \times \bar{\Omega}$, then $u$ is a subsolution of $(E)$.

8.1'. Lemma. Assume conditions (F1) and (F2). Let $S$ be a nonempty family of a supersolution of $(E)$. Let $u$ be a function defined on $Q$ by

$$
u(t, x)=\inf \{v(t, x) ; v \in S\} \quad \text { for }(t, x) \in Q .
$$

Suppose $u_{*}>-\infty$ in $[0, T) \times \bar{\Omega}$, then $u$ is a supersolution of $(E)$.

8.2. Lemma. Assume conditions (F1) and (F2). Let $h: Q \rightarrow \mathbf{R}$ be a supersolution of $(E)$. Let $S$ be the collection of all subsolution $v$ of $(E)$ with $v \leq h$ in $Q$. If $v \in S$ is not a supersolution of $(E)$ and $v_{*}>-\infty$ in $[0, T) \times \bar{\Omega}$, then there are a function $w \in S$ and a point $(s, y) \in Q$ such that $v(s, y)<w(s, y)$.

Theorem 3.3 follows from these two lemmas as in [I]. We give its proof for completeness.

Proof of Perron type Existence Theorems 3.3 and 3.4 admitting Lemmas 8.1 and 8.2.

Let $S$ be $\left\{v ; v\right.$ is a subsolution of (E) and $v \leq u^{+}$in $\left.Q\right\}$. Since $u^{-} \in S$, we see $S \neq \emptyset$. Let $u: Q \rightarrow \mathbf{R}$ be defined by

$$
u(t, x)=\sup \{v(t, x) ; v \in S\} \quad \text { for }(t, x) \in Q \text {. }
$$

By definition $u^{*} \leq\left(u^{+}\right)^{*}<\infty$ in $[0, T) \times \bar{\Omega}$. By Lemma $8.1 u$ is a subsolution of $(\mathrm{E})$, so that $u \in S$. Since $u^{-} \in S$, we have $u^{-} \leq u \leq u^{+}$in $Q$ and $-\infty<\left(u^{-}\right)_{*} \leq u_{*}$ in $[0, T) \times \bar{\Omega}$.

Suppose that $u$ were not a supersolution of (E). By Lemma 8.2 there would exist $w \in S$ and $(s, y) \in Q$ such that $u(s, y)<w(s, y)$. This contradicts the definition of $u$. Therefore $u$ is a generalized solution of (E). 
Suppose that $u$ were not $\varpi$-periodic in $x$. Then there would exist a point $\left(t_{0}, x_{0}\right) \in(0, T) \times \mathbf{R}$ and $\nu \in\{-1,1\}$ that satisfy

$$
u\left(t_{0}, x_{0}\right)<u\left(t_{0}, x_{0}+\nu \varpi\right)
$$

We observe that

$$
v(t, x)=u(t, x+\nu \varpi)
$$

belongs to $S$. Indeed, since (E) is invariant under translation in $x, v$ is a subsolution of $(\mathrm{E})$ in $(0, T) \times \mathbf{R}$. The function $u^{+}$is $\varpi$-periodic, so $v \leq u^{+}$ in $(0, T) \times \mathbf{R}$, which yields $v \in S$. By the definition of $u$ the property $v \in S$ implies $u \geq v$. This contradicts

$$
u\left(t_{0}, x_{0}\right)<v\left(t_{0}, x_{0}\right)=u\left(t_{0}, x_{0}+\nu \varpi\right) .
$$

We thus conclude $u$ is $\varpi$-periodic in $x$.

To prove Lemmas 8.1 and 8.2, we extend the method found in $[\mathrm{I}]$ to faceted functions. The basic strategy is to utilize the advantage of our definition of $C_{P}^{2}$ functions. We have arranged that the weighted curvature of a $C_{P}^{2}$ function at the boundary of a faceted region equals that at an interior point of the faceted region. Moreover, we have arranged that the weighted curvature of a $C_{P}^{2}$ function at point $y_{k}$ outside the faceted region tends to zero, if the point $y_{k}$ tends to a point $\bar{y}$ of the boundary of the faceted region as $k \rightarrow+\infty$, since the function is $C^{2}$. These properties will be invoked to estimate the weighted curvature of a modification of a test function, which we call an upper or a lower canonical modification defined below.

8.3. Notation of an upper and a lower canonical modification. Let $\Omega_{1}$ be an open interval with $\Omega_{1} \subset \Omega$. Let $f \in C(\Omega)$ satisfy $\left.f\right|_{\Omega_{1}} \in C_{P}^{2}\left(\Omega_{1}\right)$ and $f^{\prime}(\hat{x})=0$ with $\hat{x} \in \Omega_{1}$. Let $q_{1}=\sup \{p \in P \cup\{-\infty\} ; p<0\}$ and $q_{2}=\inf \{p \in P \cup\{+\infty\} ; p>0\}$.

Case(i) $(0 \notin P)$. Set

$$
f^{\#}(x)=f(x)+(x-\hat{x})^{4} \quad \text { for } x \in \Omega .
$$

There exists an open interval $\Omega_{2} \subset \Omega_{1}$ containing $\hat{\boldsymbol{x}}$ such that

$$
q_{1} / 2<\left(f^{\#}\right)^{\prime}(x)<q_{2} / 2 \quad \text { for all } x \in \Omega_{2} \text {. }
$$

Case(ii) $(0 \in P)$. We see that $f$ is $P$-faceted at $\hat{x}$ in $\Omega_{1}$. We denote $\Omega=$ $\left(a_{-}, a_{+}\right)$and $R(f, \hat{x})=\left[c_{-}, c_{+}\right]$. There exists an open interval $\Omega_{2}=\left(b_{-}, b_{+}\right)$ such that $R(f, \hat{x}) \subset \Omega_{2} \subset \Omega_{1}$ and

$$
\left\{\begin{array}{l}
f^{\prime}(x) \in\left(q_{1} / 4,0\right) \cap\left(0, q_{2} / 4\right) \quad \text { for all } x \in \Omega_{2} \backslash R(f, \hat{x}) \\
b_{+} \leq c_{+}+\left(q_{2} / 16\right)^{1 / 3} \\
b_{-} \geq c_{-}-\left(-q_{1} / 16\right)^{1 / 3}
\end{array}\right.
$$


If $\chi_{+}(f, \hat{x})=1($ resp. $\chi-(f, \hat{x})=1)$, then we set

$$
\begin{aligned}
& f^{\#}(x)= \begin{cases}f(\hat{x}) & \text { for } x \in\left[\hat{x}, c_{+}\right] \\
f(x)+\left(x-c_{+}\right)^{4} & \text { for } x \in\left(c_{+}, a_{+}\right) .\end{cases} \\
& \left(\text {resp. } f^{\#}(x)=\left\{\begin{array}{ll}
f(\hat{x}) & \text { for } x \in\left[c_{-}, \hat{x}\right), \\
f(x)+\left(x-c_{-}\right)^{4} & \text { for } x \in\left(a_{-}, c_{-}\right) .
\end{array}\right)\right.
\end{aligned}
$$

If $\chi_{+}(f, \hat{x})=-1$ (resp. $\left.\chi-(f, \hat{x})=-1\right)$ then for $\varepsilon \in\left(0, \varepsilon_{0}\right)$ we set

$$
\begin{aligned}
& f^{\#, \varepsilon}(x)= \begin{cases}f(\hat{x}) & \text { for } x \in\left[\hat{x}, c_{+}+\varepsilon\right], \\
f(x-\varepsilon) & \text { for } x \in\left(c_{+}+\varepsilon, b_{+}\right), \\
f(x)+\left\{f\left(b_{+}-\varepsilon\right)-f\left(b_{+}\right)\right\} & \text {for } x \in\left(b_{+}, a_{+}\right) .\end{cases} \\
& \left(\begin{array}{ll}
f(\hat{x}) & \text { for } x \in\left[c_{-}-\varepsilon, \hat{x}\right), \\
f(x+\varepsilon) & \text { for } x \in\left(b_{-}, c_{-}-\varepsilon\right), \\
f(x)+\left\{f\left(b_{-}+\varepsilon\right)-f\left(b_{-}\right)\right\} & \text {for } x \in\left(a_{-}, b_{-}\right] .
\end{array}\right)
\end{aligned}
$$

where $\varepsilon_{0}=\operatorname{dist}\left(\partial \Omega_{2}, R(f, \hat{x})\right) / 2$. Here we note that (8.1) holds.

We often suppress $\varepsilon$-dependence of $f^{\#, \varepsilon}$, even if it depends on $\varepsilon$. We call $f^{\#}$ an upper canonical modification of $f$ at $\hat{x}$ with canonical neighborhood $\Omega_{\mathbf{2}}$. Let $-f_{\#}$ be an upper canonical modification of $-f$ at $\hat{x}$ with canonical neighborhood $\Omega_{2}$, and we call $f_{\#}$ a lower canonical modification of $f$ at $\hat{x}$ with canonical neighborhood $\Omega_{2}$.

8.4. Proposition. Let $\Omega$ and $\Omega_{1}$ be open intervals with $\Omega_{1} \subset \Omega$. Let $f \in C(\Omega)$ satisfy $\left.f\right|_{\Omega_{1}} \in C_{P}^{2}\left(\Omega_{1}\right)$ and $f^{\prime}(\hat{z})=0$ with $\hat{x} \in \Omega_{1}$.

Suppose that $\tilde{f}$ be an upper canonical modification $f^{\#}\left(=f^{\#, c}\right)$ or a lower canonical modification $f_{\#}\left(=f_{\#, \varepsilon}\right)$ at $\hat{x}$ with canonical neighborhood $\Omega_{2} \subset$ $\Omega_{1}$. Set $s$ and $M$ as:

$$
\begin{aligned}
& s= \begin{cases}1 & \text { if } \tilde{f} \text { is an upper canonical modification, } \\
-1 & \text { if } \tilde{f} \text { is a lower canonical modification, }\end{cases} \\
& M= \begin{cases}\{\hat{x}\} & \text { if } 0 \notin P, \\
R(f, \hat{x}) & \text { if } 0 \in P .\end{cases}
\end{aligned}
$$

(i) It holds that

$$
\begin{cases}\tilde{f} \in C(\Omega),\left.\quad \tilde{f}\right|_{\Omega_{2}} \in C_{P}^{2}\left(\Omega_{2}\right), & \\ (\tilde{f})^{(n)}(x)=f^{(n)}(\hat{x}) & \text { for } x \in M \text { and } n=0,1,2, \\ s \tilde{f}>s f \quad \text { in } \Omega \backslash M, & \text { if } s \chi(f, \hat{x}) \geq 0, \\ \Lambda_{W}(\tilde{f}, \hat{x})=\Lambda_{W}(f, \hat{x}) & \text { if } s \chi(f, \hat{x})=-1 \\ s \Lambda_{W}(\tilde{f}, \hat{x})>s \Lambda_{W}(f, \hat{x}) & \end{cases}
$$


(ii) When $0 \in P$, it holds that

$$
\left\{\begin{array}{l}
\tilde{f} \text { is } P \text {-faceted at } \hat{x} \text { in } \Omega_{2} \text { with slope } 0 \\
\chi+(\tilde{f}, \hat{x})=\chi+(f, \hat{x}), \quad \chi-(\tilde{f}, \hat{x})=\chi-(f, \hat{x}) \\
L(\tilde{f}, \hat{x})=L(f, \hat{x})+\{1-s \chi(f, \hat{x})\} \varepsilon
\end{array}\right.
$$

(iii) For any $\varepsilon_{1}>0$ and $\varepsilon_{2}>0$, there exists an open interval $\Omega_{3}$ such that

$$
\begin{cases}M \subset \Omega_{3} \subset \Omega_{2}, & \text { for } x \in \Omega_{3}, \\ \left|(\tilde{f})^{\prime}(x)\right|<\varepsilon_{1} & \text { for } x \in \Omega_{3} . \\ \left|\Lambda_{W}(\tilde{f}, x)-\Lambda_{W}(f, \hat{x})\right|<\varepsilon_{2}\end{cases}
$$

Proof of Lemma 8.1.

Step 1. Let $(\hat{t}, \hat{x}) \in Q$. Let $\varphi \in C(\Omega)$ be locally admissible at $(\hat{t}, \hat{x})$ in $Q$. Suppose that

$$
\max _{Q}\left(u^{*}-\varphi\right)=\left(u^{*}-\varphi\right)(\hat{t}, \hat{z})
$$

Our goal is to show the inequality :

$$
\varphi_{t}(\hat{t}, \hat{x})+F\left(\hat{t}, \varphi_{x}(\hat{t}, \hat{z}), \Lambda_{W}(\varphi(\hat{t}, \cdot), \hat{z})\right) \leq 0
$$

(cf. Theorem 6.8). Without loss of generality we may assume that ( $u^{*}-$ $\varphi)(\hat{t}, \hat{x})=0$, since $\varphi(t, x)$ can be replaced by $\varphi(t, x)+\left(u^{*}-\varphi\right)(\hat{t}, \hat{x})$. We may assume that $\varphi_{x}(\hat{t}, \hat{x})=0$ by Proposition 2.7 with $A=\varphi_{x}(\hat{t}, \hat{x})$ and $B=-\varphi_{\mathfrak{x}}(\hat{t}, \hat{x}) \hat{x}$.

Since $\varphi$ is locally admissible at $(\hat{t}, \hat{x})$ in $Q$, there exists a rectangular neighborhood $Q_{1}=I \times \Omega_{1}$ at $(\hat{t}, \hat{x})$ in $Q$ such that $\left.\varphi\right|_{Q_{1}} \in A_{P}\left(Q_{1}\right)$. So there exist $f \in C_{P}^{2}\left(\Omega_{1}\right)$ and $g \in C^{1}(I)$ such that

$$
\varphi(t, x)=f(x)+g(t) \quad \text { for }(t, x) \in Q_{1} .
$$

The inequality (8.3) becomes

$$
g^{\prime}(\hat{t})+F\left(\hat{t}, 0, \Lambda_{W}(f, \hat{x})\right) \leq 0
$$

which we should prove.

Let $\zeta$ be a function on $Q$ satisfying

$$
\left\{\begin{array}{l}
\zeta \in C(Q), \quad \zeta(\hat{t}, \hat{x})=0, \quad \zeta \geq 0 \quad \text { in } Q \\
\{(t, x) \in Q ; \zeta(t, x)=0\} \cap \bar{\theta}_{p} Q=\emptyset
\end{array}\right.
$$


where $\bar{\partial}_{p} Q=[0, T] \times \theta \Omega \cap\{0\} \times \bar{\Omega}$. The function $\zeta$ is to be determined later. Setting $\psi=\varphi+\zeta$ in $Q$, we see

$$
\max _{Q}\left(u^{*}-\psi\right)=\left(u^{*}-\psi\right)(\hat{t}, \hat{x})
$$

so that

$\left(u^{*}-\psi\right)(t, x)+\zeta(t, x)=\left(u^{*}-\varphi\right)(t, x) \leq\left(u^{*}-\varphi\right)(\hat{t}, \hat{x})=0 \quad$ for $(t, x) \in Q$,

which implies

$$
\left(u^{*}-\psi\right) \leq-\zeta \quad \text { in } Q .
$$

By the definition of the uppersemicontinuous envelope, there exists a sequence

$\left\{\left(t_{k}, x_{k}\right)\right\}_{k=1}^{\infty} \subset Q$ such that $\left(t_{k}, x_{k}\right) \rightarrow(\hat{t}, \hat{x})$ as $k \rightarrow \infty$ and

$$
\lim _{h \rightarrow \infty}\left(u^{*}-\psi\right)\left(t_{h}, x_{k}\right)=\left(u^{*}-\psi\right)(\hat{t}, \hat{x})=0
$$

By the definition of $u$, there is a sequence $\left\{v_{k}\right\}_{k=1}^{\infty} \subset S$ such that $v_{k}\left(t_{k}, x_{k}\right)>$ $u\left(t_{k}, x_{k}\right)-1 / k$. So there is a sequence $\left\{\left(s_{k}, y_{k}\right)\right\}_{k=1}^{\infty} \subset \bar{Q}$ such that

$$
\max _{\bar{Q}}\left(v_{k}^{*}-\psi\right)=\left(v_{k}^{*}-\psi\right)\left(s_{k}, y_{k}\right)
$$

These yield

$$
\begin{aligned}
& u^{*}\left(t_{k}, x_{k}\right)-1 / k-\psi\left(t_{k}, x_{k}\right)<\left(v_{k}^{*}-\psi\right)\left(t_{k}, x_{k}\right) \\
& \leq\left(v_{k}^{*}-\psi\right)\left(s_{k}, y_{k}\right) \leq\left(u^{*}-\psi\right)\left(s_{k}, y_{k}\right) \leq-\zeta\left(s_{k}, y_{k}\right)
\end{aligned}
$$

so that $\lim _{k \rightarrow \infty} \zeta\left(s_{k}, y_{k}\right)=0$ since the leftest hand side of the last inequality converges to 0 as $k$ tends to $+\infty$. So we get $\lim _{k \rightarrow \infty}\left(u^{*}-\psi\right)\left(s_{k}, y_{k}\right)=0$ and $(\bar{s}, \bar{y}) \in\{(t, x) \in Q ; \zeta(t, x)=0\}$, where $(\bar{s}, \bar{y})=\lim _{k \rightarrow \infty}\left(s_{k}, y_{k}\right)$ by taking a subsequence. Since the zero set of $\zeta$ does not intersect $\bar{\partial}_{P} Q$, for sufficiently large $k>0$ we have $\left(s_{k}, y_{k}\right) \in Q$ and

$$
\max _{Q}\left(v_{k}^{*}-\psi\right)=\left(v_{k}^{*}-\psi\right)\left(s_{k}, y_{h}\right)
$$

Since $v_{h}$ is a subsolution of $(\mathrm{E})$, we have

$$
\psi_{t}\left(s_{k}, y_{k}\right)+F\left(s_{k}, \psi_{z}\left(s_{k}, y_{k}\right), \Lambda_{W}\left(\psi\left(s_{k}, \cdot\right), y_{k}\right)\right) \leq 0
$$

provided that $\psi$ is locally admissible near $\left(s_{k}, y_{k}\right)$ (Proposition 6.8). 
Step 2. Let $f^{\#} \in C(\Omega)$ be an npper canonical modification of $f$ at $\hat{z}$ with canonical neighborhood $\Omega_{2} \subset \Omega_{1}$, so that $\left.f^{\#}\right|_{\Omega_{2}} \in C_{P}^{2}\left(\Omega_{2}\right)$ by Proposition 8.4 (i). We choose $\zeta(t, x)=\eta(t)+\left\{f^{\#}(x)-f(x)\right\}$ for $(t, x) \in Q$ with $\eta(t)=$ $(t-\hat{t})^{2}$, so that (8.5) holds since zero set of $\zeta$ is $\{\hat{t}\} \times M$ from Proposition 8.4(i). Then we observe that $\bar{s}=\hat{t}$ and

$$
\psi(t, x)=g(t)+\eta(t)+f^{\#}(x) \quad \text { for }(t, x) \in Q_{2}=I \times \Omega_{2},
$$

which belongs to $A_{P}\left(Q_{2}\right)$. Inequality (8.6) becomes

$$
g^{\prime}\left(s_{k}\right)+\eta^{\prime}\left(s_{k}\right)+F\left(s_{k},\left(f^{\#}\right)^{\prime}\left(y_{k}\right), \Lambda_{W}\left(f^{\#}, y_{k}\right)\right) \leq 0 .
$$

Case(i) ( $f$ is not $P$-faceted at $\hat{z}$ in $\Omega$ ). By Proposition 8.4 (ii), we see $\bar{y}=\hat{\boldsymbol{x}}$ and $\left(f^{\#}\right)^{(n)}(\hat{x})=f^{(n)}(\hat{x})$ for $n=0,1,2$. Sending $k$ to $+\infty$ in inequality (8.7), we conclude (8.4) since we assumed continuity (F1) and $\psi$ is the first, and second continuously differentiable with respect to $t$, and $x$, respectively. Case(ii) ( $f$ is $P$-faceted at $\hat{x}$ in $\Omega$ and $\chi(f, \hat{x}) \geq 0$ ). Let $I^{\prime}$ be an open interval containing $\hat{t}$ with $\overline{I^{\top}} \subset I$. We see $\{(t, x) \in \Omega ; \zeta(t, x)=0\}=\{\hat{t}\} \times R(f, \hat{x})$.

(A) Suppose that there exists a sequence $\left\{k_{j}\right\}_{j=1}^{\infty}$ such that $\left(s_{h_{j}}, y_{h_{j}}\right) \in$ $\overline{I^{\prime}} \times R(f, \hat{x})$, so that $f^{\#}$ is $P$-faceted at $y_{k_{j}}$ in $\Omega_{2}$ with slope 0 . Owing to the definition of weighted curvature at the boundary of a faceted region, $\Lambda_{W}\left(f^{\#}, y_{h_{j}}\right)=\Lambda_{W}(f, \hat{x})$ by Proposition 8.4 (i). So (8.7) becomes

$$
g^{\prime}\left(s_{k_{j}}\right)+\eta^{\prime}\left(s_{k_{j}}\right)+F\left(s_{k_{j}}, 0, \Lambda_{W}\left(f^{\#}, \hat{x}\right)\right) \leq 0 .
$$

Sending $j$ to $+\infty$, we have (8.4) by continuity assumption (F1).

(B) Consider the negation of (A): for all $k>0,\left(s_{k}, y_{k}\right) \in\left\{I \backslash \overline{I^{\prime}}\right\} \times$ $\left\{\Omega_{2} \backslash R(f, \hat{x})\right\}$. We observe that $\bar{y} \in \partial R(f, \hat{x})$. Setting

$$
\lambda(y)=W^{\prime \prime}\left(\left(f^{\#}\right)^{\prime}(y)\right)\left(f^{\#}\right)^{\prime \prime}(y) \quad \text { for } y \in \Omega_{2},
$$

we have $\Lambda_{W}\left(f^{\#}, y_{k}\right)=\lambda\left(y_{k}\right)$ since $f^{\#}$ is not $P$-faceted at $y_{k}$ in $\Omega_{2}$. We see $\lim _{k \rightarrow+\infty}\left(f^{\#}\right)^{\prime}\left(y_{k}\right)=0=\lim _{k \rightarrow+\infty}\left(f^{\#}\right)^{\prime \prime}\left(y_{k}\right)$ by $f^{\#} \in C^{2}\left(\Omega_{2}\right)$, so that $\lim _{k \rightarrow+\infty} \lambda\left(y_{k}\right)=0$ since $W^{\prime \prime}$ is bounded on every bounded set in $R \backslash P$. Since inequality (8.7) becomes

$$
g^{\prime}\left(s_{k}\right)+\eta^{\prime}\left(s_{k}\right)+F\left(s_{k},\left(f^{\#}\right)^{\prime}\left(y_{k}\right), \lambda\left(y_{k}\right)\right) \leq 0,
$$

we have

$$
g^{\prime}(\hat{t})+F(\hat{t}, 0,0) \leq 0
$$

by sending $k$ to infinity. Since assumption $\chi(f, \hat{z}) \geq 0$ yields $\Lambda_{W}(f, \hat{z}) \geq 0$, we now get (8.4) by degenerate ellipticity assumption (F2). 
Case(iii) ( $f$ is $P$-faceted at $\hat{x}$ in $\Omega$ and $\chi(f, \hat{x})=-1$ ). Fix $\varepsilon \in\left(0, \varepsilon_{0}\right)$, where $\varepsilon_{0}$ is defined in 8.3. Since $(\bar{s}, \bar{y}) \in\{\hat{t}\} \times R(f, \hat{x})$ and $R(f, \hat{x}) \subset$ int $R\left(f^{\#, \varepsilon}, \hat{x}\right)$, there exists $k_{0}>0$ such that $\left(s_{k}, y_{k}\right) \in I \times R\left(f^{\#, \varepsilon}, \hat{x}\right)$ for all $k>k_{0}$. For $\left(s_{k}, y_{k}\right) \in I \times R\left(f^{\#, \varepsilon}, \hat{x}\right),(8.7)$ becomes

$$
g^{\prime}\left(s_{k}\right)+\eta^{\prime}\left(s_{k}\right)+F\left(s_{k}, 0,-\Delta /(L+2 \varepsilon)\right) \leq 0,
$$

where $\Delta=W^{\prime}(+0)-W^{\prime}(-0)$ and $L=L(f, \hat{x})$. Taking $k$ to $+\infty$, we get

$$
g^{\prime}(\hat{t})+F(\hat{t}, 0,-\Delta /(L+2 \varepsilon)) \leq 0 .
$$

Since the last inequality holds for all $\varepsilon \in\left(0, \varepsilon_{0}\right)$, we get (8.4).

Lemma 8.1' is proved parallelly as Lemma 8.1.

Proof of Lemma 8.2.

Step 1. Let $(\hat{t}, \hat{x}) \in Q$ and let $\varphi \in C(\Omega)$ be locally admissible at $(\hat{t}, \hat{x})$ in $Q$. Suppose that

$$
\min _{Q}\left(v^{*}-\varphi\right)=\left(v^{*}-\varphi\right)(\hat{t}, \hat{x})
$$

Without loss of generality we may assume that $\left(v_{*}-\varphi\right)(\hat{t}, \hat{x})=0$ and we may also assume that $\varphi_{x}(\hat{t}, \hat{x})=0$ by Proposition 2.7.

Let $\zeta$ be a function on $Q$ satisfying

$$
\left\{\begin{array}{l}
\zeta \in C(Q), \quad \zeta(\hat{t}, \hat{x})=0, \quad \zeta \geq 0 \text { in } Q, \\
\psi \text { is a locally admissible function at }(\hat{t}, \hat{x}) \text { in } Q,
\end{array}\right.
$$

where $\psi=\varphi-\zeta$ in $Q$. Function $\zeta$ is to be determined later. So we see

$$
\min _{Q}\left(v^{*}-\psi\right)=\left(v^{*}-\psi\right)(\hat{t}, \hat{x})
$$

and

$$
\zeta \leq v_{*}-\psi \quad \text { in } Q .
$$

Suppose that by choosing $\zeta$ suitably there exists a rectangular neighborhood $N_{1}$ at $(\hat{t}, \hat{x})$ in $Q$ satisfying

$$
\psi_{t}(t, x)+F\left(t, \psi_{x}(t, x), \Lambda_{W}(\psi(t, \cdot), x)\right)<0 \quad \text { for all }(t, x) \in N_{1},
$$

$$
\left.\psi\right|_{N_{1}} \in A_{P}\left(N_{1}\right)
$$


and

$$
N_{0} \subset N_{1} \quad \text { with } N_{0}=\{(t, x) \in Q ; \zeta(t, x)=0\} .
$$

Let $N_{2}$ be a rectangular (open) neighborhood at $(\hat{t}, \hat{z})$ satisfying $N_{0} \subset N_{2}$ and $\overline{N_{2}} \subset N_{1}$. There exists $\sigma_{1}>0$ such that

$$
\psi+\sigma_{1}<h_{*} \quad \text { in } N_{2}
$$

In fact, from (8.9) and the definition of $S, \psi \leq v_{*} \leq h_{*}$ in $Q$. If there exists $\left(t_{1}, x_{1}\right) \in \overline{N_{2}}$ such that $\psi\left(t_{1}, x_{1}\right)=h_{*}\left(t_{1}, x_{1}\right)$ then locally admissible function $\psi$ is a test function of $h$ at $\left(t_{1}, x_{1}\right)$ in $Q$, which contradicts (8.10). So we have $\psi<h_{*}$ in $N_{2}$, or there exists $\sigma_{1}>0$ such that (8.13).

Since $\sigma_{2}=\inf \left\{\zeta(x) ; x \in N_{1} \backslash N_{2}\right\}>0$ by the definition of $N_{2}$, we have

$$
\psi+\sigma_{2} \leq v_{*} \quad \text { in } N_{1} \backslash N_{2}
$$

which yields

$$
\psi+\sigma \leq h_{*} \quad \text { in } N_{1}
$$

with $\sigma=\min \left(\sigma_{1}, \sigma_{2}\right)$. By Propositions 2.7 and 2.8 we conclude that $\psi+\sigma$ is a subsolution of (E) in $N_{1}$.

We define $w(t, x)$ by

$$
w(t, x)= \begin{cases}\max \{\psi(t, x)+\sigma, v(t, x)\} & (t, x) \in N_{2} \\ v(t, x) & (t, x) \in Q \backslash N_{2}\end{cases}
$$

Inequality (8.14) yields

$$
w(t, x)=\max \{\psi(t, x)+\sigma, v(t, x)\} \quad \text { for }(t, x) \in N_{1} \text {. }
$$

So $w$ is a subsolution of $(\mathrm{E})$ in $N_{1}$ by Lemma 8.1'.

To show $w$ is a subsolution of (E) in $Q$ suppose that $\psi_{1} \in A_{P}(Q)$ fulfills

$$
\max _{Q}\left(w-\psi_{1}\right)=\left(w-\psi_{1}\right)\left(t_{0}, x_{0}\right)=0
$$

We may assume that

$$
\left(t_{0}, x_{0}\right) \in N_{2} \text { and } \psi_{1}\left(t_{0}, x_{0}\right)>v\left(t_{0}, x_{0}\right)
$$


since otherwise $\psi_{1}$ is a test function of $v$ at $\left(t_{0}, x_{0}\right)$ so that

$$
\left(\psi_{1}\right)_{t}\left(t_{0}, x_{0}\right)+F\left(t_{0},\left(\psi_{1}\right)_{x}\left(t_{0}, x_{0}\right), \Lambda_{W}\left(\psi_{1}\left(t_{0}, \cdot\right), x_{0}\right)\right) \leq 0
$$

We may assume that $\psi_{1}\left(t_{0}, \cdot\right)$ is faceted at $x_{0}$ with slope $\left(\psi_{1}\right)_{x}\left(t_{0}, \cdot\right) \in P$ and $R=R\left(\psi_{1}\left(t_{0}, \cdot\right), x_{0}\right)$ is not included in an interval $J_{1}$ with $N_{1}=J_{1} \times I_{1}$. Indeed, if not, (2.1') holds by Proposition 6.19 since $w$ is a subsolution of (E) in $N_{1}$. We may also assume that

$$
\psi_{1}\left(t_{0}, x\right)>v\left(t_{0}, x\right) \quad \text { for } x \in R \cap J_{1} \text {. }
$$

Indeed, if not there is $x_{1} \in R \cap J_{1}$ with $\psi_{1}\left(t_{0}, x_{1}\right) \leq v\left(t_{0}, x_{1}\right)$. Since $v \leq w \leq$ $\psi_{1}$ in $Q$, we now observe that

$$
\max _{Q}\left(v-\psi_{1}\right)=\left(v-\psi_{1}\right)\left(t_{0}, x_{1}\right)
$$

which yields (2.1') with $x_{0}$ replaced by $x_{1}$. Since $v$ is a subsolution of (E) in $Q$ and since $x_{1} \in R$ implies $\left(\psi_{1}\right)_{x}\left(t_{0}, x_{0}\right)=\left(\psi_{1}\right)_{x}\left(t_{0}, x_{1}\right)$ we now have $\left(2,1^{\prime}\right)$ without replacing $x_{0}$ by $x_{1}$. We may now assume that

$$
\psi_{1}(t, x)>v(t, x) \quad \text { on } N_{1}, x \in R \cap J_{1}
$$

by taking $I_{1}$ smaller since $v$ is uppersemicontinuous. We now modify $\psi_{1}\left(t_{0}, \cdot\right)$ in $R \cap\left(J_{1} \backslash J_{2}\right)$ with $N_{2}=J_{2} \times I_{2}$ to get $\psi_{2} \in A_{P}\left(N_{1}\right)$ satisfying

$$
\begin{aligned}
& \max _{N_{1}}\left(w-\psi_{2}\right)=\left(w-\psi_{2}\right)\left(t_{0}, x_{0}\right), \\
& \text { int } R\left(\psi_{2}\left(t_{0}, \cdot\right), x_{0}\right) \subset N_{1}, \\
& \Lambda_{W}\left(\psi_{2}\left(t_{0}, \cdot\right), x_{0}\right) \leq \Lambda_{W}\left(\psi_{1}\left(t_{0}, \cdot\right), x_{0}\right), \\
& \psi_{1}=\psi_{2} \quad \text { in } N_{2} .
\end{aligned}
$$

Since $w$ is a subsolution in $N_{1}$ (even for new choice of $I_{1}$ by Proposition 6.19) this yields $\left(2,1^{\prime}\right)$ with $\psi_{1}$ replaced by $\psi_{2}$. By (F2) we now have (2.1'). By (8.15) we now conclude that $w$ is a subsolution of (E) in $Q$ and $w \in S$.

On the other hand, we have

$0=\left(v_{*}-\psi\right)(\hat{t}, \hat{x})=\liminf _{d \downarrow 0}\left\{(v-\psi)(t, x) ;(t, x) \in N_{2},|t-\hat{t}|<d,|x-\hat{x}|<d\right\}$

which implies that there exists $(s, y) \in N_{2}$ such that $v(s, y)-\psi(s, y)<\sigma$. We now obtain $v(s, y)<w(s, y)$.

Step 2. We shall prove that there exists $\zeta$ satisfying (8.8) and $N_{1}$ satisfying $(8.10),(8.11)$ and (8.12). 
Since $\varphi$ is locally admissible at $(\hat{t}, \hat{x})$ in $Q$, there exists a rectangular neighborhood $Q_{1}=I \times \Omega_{1}$ at $(\hat{t}, \hat{x})$ in $Q$ such that $\left.\varphi\right|_{Q_{1}} \in A_{P}\left(Q_{1}\right)$. So there exists $f \in C_{P}^{2}\left(\Omega_{1}\right)$ and $g \in C^{1}(I)$ such that

$$
\varphi(t, x)=f(x)+g(t) \quad \text { for }(t, x) \in Q_{1} .
$$

Let $f_{\#} \in C(\Omega)$ be a lower canonical modification of $f$ at $\hat{\boldsymbol{x}}$ with canonical neighborhood $\Omega_{2} \subset \Omega_{1}$. We chose $\zeta(t, x)=\eta(t)+\left\{f(x)-f_{\#}\right\}(\geq 0)$ for $(t, x) \in Q$ with $\eta(t)=(t-\hat{t})^{2}$, so that

$$
\psi(t, x)=g(t)-\eta(t)+f_{\#}(x) \quad \text { for }(t, x) \in N_{3}=I \times \Omega_{2} .
$$

Proposition 8.4 (i) yields (8.8) and $N_{0}=\{\hat{t}\} \times M$, where $M$ is the same as (8.2).

Since $v$ is not a supersolution of $(E)$ and $v_{*}>-\infty$ in $[0, T) \times \bar{\Omega}$, we have

$$
\psi_{t}(\hat{t}, \hat{x})+F\left(\hat{t}, \psi_{z}(\hat{t}, \hat{z}), \Lambda_{W}(\psi(\hat{t}, \cdot), \hat{x})\right)<0
$$

or

$$
g^{\prime}(\hat{t})-\eta^{\prime}(\hat{t})+F\left(\hat{t}, 0, \Lambda_{W}\left(f_{\#}, \hat{x}\right)\right)<0
$$

by the definition of supersolution of local version 6.7 and Theorem 6.8 . Clearly, there exists $\delta>0$ such that

$$
g^{\prime}(\hat{t})-\eta^{\prime}(\hat{t})+F\left(\hat{t}, 0, \Lambda_{W}\left(f_{\#}, \hat{x}\right)\right)<-\delta .
$$

For $(t, x) \in N_{3}$ we have

$$
\begin{aligned}
& g^{\prime}(t)+F\left(t,\left(f_{\#}\right)^{\prime}(x), \Lambda_{W}\left(f_{\#}, x\right)\right) \\
& \left.<g^{\prime}(t)-g^{\prime}(\hat{t})+\eta^{\prime}(\hat{t})\right\} \\
& \quad+\left\{F\left(t,\left(f_{\#}\right)^{\prime}(x), \Lambda_{W}\left(f_{\#}, x\right)\right)-F\left(\hat{t}, 0, \Lambda_{W}(f, \hat{x})\right)\right\} \\
& \quad+\left\{F\left(\hat{t}, 0, \Lambda_{W}(f, \hat{x})\right)-F\left(\hat{t}, 0, \Lambda_{W}\left(f_{\#}, \hat{x}\right)\right)\right\} \\
& \quad-\delta .
\end{aligned}
$$

Let $T_{1}, T_{2}$ and $T_{3}$ denote the first, second and third term of the right hand side of the last inequality. We shall show that there exists an open set $N_{1}$ such that $N_{0} \subset N_{1} \subset N_{3}$ and for all $(t, x) \in N_{1}, T_{1}+T_{2}+T_{3}-\delta<0$ holds. This now yields

$$
g^{\prime}(t)+F\left(t,\left(f_{\#}\right)^{\prime}(x), \Lambda_{W}\left(f_{\#}, x\right)\right)<0 \quad \text { for }(t, x) \in N_{1},
$$

which equals (8.10). 
By Proposition 8.4(i), we see $\Lambda_{W}\left(f_{\#}, \hat{x}\right) \leq \Lambda_{W}(f, \hat{x})$, so that

$$
T_{3} \leq 0
$$

holds by degenerate ellipticity (F2). Since $g, \eta \in C^{1}(I)$, there exists $\rho_{1} \in$ $(0, \operatorname{dist}(\hat{t}, \partial I))$ such that

$$
T_{1}=g^{\prime}(t)-g^{\prime}(\hat{t})+\eta^{\prime}(\hat{t})<\delta / 2 \quad \text { for all } t \in B\left(\hat{t}, \rho_{1}\right),
$$

where $B\left(\hat{t}, \rho_{1}\right)$ denotes an open ball in $\mathbf{R}$ with center $\hat{t}$ and radius $\rho_{1}$. From continuity (F1), there exists $\rho_{2}>0$ such that

$$
|F(t, p, X)-F(\hat{t}, 0,0)|<\delta / 2 \quad \text { for all } t, p \text { and } X \in B\left(0, \rho_{2}\right) \text {. }
$$

From Proposition 8.4 (iii), there exists a open interval $\Omega_{3}$ such that

$$
\begin{cases}M \subset \Omega_{3} \subset \Omega_{2}, & \text { for } x \in \Omega_{3}, \\ \left|\left(f_{\#}\right)^{\prime}(x)\right|<\rho_{2} & \text { for } x \in \Omega_{3} .\end{cases}
$$

We chose $N_{1}=B\left(\hat{t}, \min \left(\rho_{1}, \rho_{2}\right)\right) \times \Omega_{3}$, so that (8.11) and (8.12) hold. Then we get $T_{2}<\delta / 2$ for $(t, x) \in N_{1}$. We now have

$$
T_{1}+T_{2}+T_{3}-\delta<0 \quad \text { for }(t, x) \in N_{1} \text {, }
$$

so that we conclude (8.10) for $(t, x) \in N_{1}$.

§9. Existence Theorem for periodic initial data.

We shall give the proof of Existence Theorem for periodic initial data 3.5. Throughout this section, let $T>0, S \in(0, T)$ and $Q_{s}=(0, S) \times \mathbf{R}$. We often use the condition:

$$
F \text { is continuous in }[0, S] \times \mathbf{R} \times \mathbf{R} \text { with values } \mathbf{R} \text {. }
$$

The key tool to prove the Existence Theorem is Comparison Theorem 3.1 and Perron type Existence Theorem 3.4 with the following Lemma:

9.1. Lemma (Existence of super- and subsolution). Assume conditions (F1') and (F2). Suppose that $u_{0}$ is bounded and uniformly continuous in R. Then for each $S \in(0, T)$ there exists an uppersemicontinuous function 
$u^{+}\left(=u^{+, s}\right)$ (resp. a lowersemicontinuous function $u^{-}\left(=u^{-, s}\right)$ ) on $\overline{Q_{S}}$ such that $u^{+}$(resp. $u^{-}$) is a super- (resp. sub-) solution of $(E)$ in $Q_{s}$ and

$$
\begin{aligned}
& \begin{cases}u^{+}(0, x)=u_{0}(x) & \text { for } x \in \mathbf{R}, \\
u^{+}(t, x) \geq u_{0}(x) & \text { for }(t, x) \in \overline{Q_{s}} .\end{cases} \\
& \left(\begin{array}{ll}
\text { resp. }\left\{\begin{array}{ll}
u^{-}(0, x)=u_{0}(x) & \text { for } x \in \mathbf{R}, \\
u^{-}(t, x) \leq u_{0}(x) & \text { for }(t, x) \in \overline{Q_{s}}
\end{array}\right)
\end{array}\right.
\end{aligned}
$$

9.2. Remark. If $u_{0}$ is periodic with period $\varpi$, the lemma holds with extra property

$$
\begin{aligned}
& u^{+}(t, x+\varpi)=u^{+}(t, x) \quad \text { for }(t, x) \in \overline{Q_{s}} \\
& \left(\text { resp. } \quad u^{-}(t, x+\varpi)=u^{-}(t, x) \quad \text { for }(t, x) \in \overline{Q_{s}}\right)
\end{aligned}
$$

To prove Lemma 9.1, we extend the method developed in [CGG] and [IS] to $C_{P}^{2}$ functions. We shall prove in several steps.

9.3. Proposition. Let $M$ be a positive number. For any $\delta$ there exists $f_{\delta}\left(=f_{\delta}^{M}\right) \in C_{P}^{2}(\mathbf{R})$ such that

$$
\begin{cases}f_{\delta}(0)=0, & \\ f_{\delta}, f_{\delta}^{\prime \prime} \geq 0 & \text { in } \mathbf{R}, \\ f_{\delta}(x) \geq M & \text { for } x,|x|>\delta\end{cases}
$$

Proof. We set

$$
V_{0}(x)= \begin{cases}(x+1)^{4} & \text { for } x<-1 \\ 0 & \text { for }-1 \leq x \leq 1 \\ (x-1)^{4} & \text { for } 1<x\end{cases}
$$

By Lemma 6.12, there exists $V_{1} \in C_{P}^{2}(\mathbf{R})$ that

$$
V_{1}(0)=0 \quad \text { and } \quad V_{1}^{\prime \prime} \geq 0, V_{1} \geq V_{0} \quad \text { in } \mathbf{R} .
$$

Clearly there exists $k=k(\delta) \in(0,1 / 2)$ with $k V_{0}(\delta / k) \geq M$. Setting $f_{\delta}(x)=$ $k V_{1}(x / k)$ for $x \in \mathbf{R}$, we see that $f_{\delta} \in C_{P}^{2}(\mathbf{R})$ since $f_{\delta}^{\prime}(x)=V_{1}^{\prime}(x / k)$ and $V_{1}$ is $P$-faceted at $\hat{\boldsymbol{x}} / \boldsymbol{k}$ in $\mathbf{R}$ if and only if $f_{\delta}$ is $P$-faceted at $\hat{\boldsymbol{x}}$ in $\mathbf{R}$. Other properties are easy to prove. 
9.4. Lemma (Modification of $C_{P}^{2}$ functions). Let $\Omega_{1}=\left(a_{1}, a_{1}^{\prime}\right), \Omega_{2}=$ $\left(a_{2}, a_{2}^{\prime}\right)$ and $\Omega_{3}$ be (nonempty) open intervals with $\overline{\Omega_{3}} \subset \Omega_{2}$ and $\overline{\Omega_{2}} \subset \Omega_{1}$. Suppose that $f \in C_{P}^{2}\left(\Omega_{1}\right)$ satisfy $f^{\prime \prime} \geq 0$ in $\Omega_{2}$. Then there exists $V \in$ $C_{P}^{2}\left(\Omega_{1}\right)$ such that

$$
\begin{cases}V^{\prime \prime} \geq 0 & \text { for } \Omega_{1}, \\ V=f & \text { for } \Omega_{3}, \\ V^{\prime}(x)= \begin{cases}q, & \text { for } x \in\left(a_{1}, a_{2}\right], \\ q^{\prime}, & \text { for } x \in\left[a_{2}^{\prime}, a_{1}^{\prime}\right) .\end{cases} \end{cases}
$$

with some $q$ and $q^{\prime} \notin P$. In particular, the number of faceted regions of $V$ is finite.

Proof. We denote $\Omega_{3}=\left(a_{3}, a_{3}^{\prime}\right)$. If there exists an open interval $I=\left(b_{1}, b_{2}\right)$ with

$$
\left\{\begin{array}{l}
\bar{I} \subset\left(a_{2}, a_{3}\right), \\
f^{\prime}(x) \notin P
\end{array} \text { for all } x \in I,\right.
$$

then we set $q=f^{\prime}\left(b_{1}\right)$. Otherwise we have $q_{1}=f^{\prime}(x) \in P$ for all $x \in\left(a_{2}, a_{3}\right)$. In the latter case we choose an open interval $I$ with $\bar{I} \subset\left(a_{2}, a_{3}\right)$ and choose $q \in\left(\sup \left\{q_{2} \in P \cap\{-\infty\} ; q_{2}<q_{1}\right\}, q_{1}\right)$.

Parallelly, if there exists an open interval $I^{\prime}=\left(b_{2}^{\prime}, b_{1}^{\prime}\right)$ with

$$
\left\{\begin{array}{l}
\overline{I^{\prime}} \subset\left(a_{3}^{\prime}, a_{2}^{\prime}\right), \\
f^{\prime}(x) \notin P \quad \text { for all } x \in I^{\prime},
\end{array}\right.
$$

then we set $q^{\prime}=f^{\prime}\left(b_{1}^{\prime}\right)$. Otherwise we have $q_{1}^{\prime}=f^{\prime}(x) \in P$ for all $x \in\left(a_{3}^{\prime}, a_{2}^{\prime}\right)$. In the latter case we choose an open interval $I^{\prime}$ with $\overline{I^{\prime}} \subset\left(a_{3}^{\prime}, a_{2}^{\prime}\right)$ and choose $q^{\prime} \in\left(q_{1}^{\prime}, \inf \left\{q_{2}^{\prime} \in P \cup\{+\infty\} ; q_{2}^{\prime}>q_{1}^{\prime}\right\}\right)$.

Let $h_{1} \in C^{1}\left(\Omega_{1}\right)$ with

$$
h_{1}(x)= \begin{cases}q & \text { for } x \in\left(a_{1}, b_{2}\right), \\ q^{\prime} & \text { for } x \in\left(b_{2}^{\prime}, a_{1}^{\prime}\right) .\end{cases}
$$

We connect $f^{\prime}$ and $h_{1}$ in the following way. Let $\rho_{1}, \rho_{2} \in C^{1}\left(\Omega_{1}\right)$ satisfy

$$
\begin{cases}\rho_{1}+\rho_{2}=1 & \text { on } \Omega_{1}, \\ 0 \leq \rho_{1}, \rho_{2} \leq 1 & \text { on } \Omega_{1},\end{cases}
$$

and

$$
\begin{aligned}
& \rho_{1}(x)=\left\{\begin{array}{ll}
1 & \text { for } x \in \overline{I_{2}}, \\
0 & \text { for } x \in \Omega_{1} \backslash I_{1},
\end{array} \quad \rho_{1}^{\prime}(x)= \begin{cases}\geq 0 & \text { for } x \in\left(b_{1}, b_{2}\right), \\
\leq 0 & \text { for } x \in\left(b_{2}^{\prime}, b_{1}^{\prime}\right),\end{cases} \right. \\
& \rho_{2}(x)=\left\{\begin{array}{ll}
0 & \text { for } x \in \overline{I_{2}}, \\
1 & \text { for } x \in \Omega_{1} \backslash I_{1},
\end{array} \quad \rho_{2}^{\prime}(x)= \begin{cases}\leq 0 & \text { for } x \in\left(b_{1}, b_{2}\right), \\
\geq 0 & \text { for } x \in\left(b_{2}^{\prime}, b_{1}^{\prime}\right),\end{cases} \right.
\end{aligned}
$$


where we denote $I_{1}=\left(b_{1}, b_{1}^{\prime}\right)$ and $I_{2}=\left(b_{2}, b_{2}^{\prime}\right)$, so that $\overline{I_{2}} \subset I_{1}$.

We set

$$
h_{2}=\rho_{1} f^{\prime}+\rho_{2} h_{1} \quad \text { in } \Omega_{1}
$$

to get

$$
h_{2}^{\prime}=\rho_{2}^{\prime}\left(h_{1}-f^{\prime}\right)+\rho_{1} f^{\prime \prime} \geq 0 \quad \text { in } \Omega_{1}
$$

since

$$
\begin{cases}h_{1} \leq f^{\prime} & \text { in }\left(b_{1}, b_{2}\right), \\ h_{1} \geq f^{\prime} & \text { in }\left(b_{2}^{\prime}, b_{1}^{\prime}\right) .\end{cases}
$$

Thus we get the desired function $V \in C_{P}^{2}\left(\Omega_{1}\right)$ by setting

$$
V(x)=f(a)+\int_{a}^{x} h_{2}(y) d y \quad \text { for } x \in \Omega_{1}
$$

with some $a \in \Omega_{3}$.

9.5. Lemma. Let $M$ be a positive number.

(i) For any $\delta \in(0,1 / 2)$ there exists $V_{\delta}\left(=V_{\delta}^{M}\right) \in C_{P}^{2}(\mathbf{R})$ such that

$$
\left\{\begin{array}{l}
V_{\delta}(0)=0, \\
V_{\delta}, V_{\delta}^{\prime \prime} \geq 0 \quad \text { in } \mathbf{R}, \\
V_{\delta} \geq M \quad \text { for } x,|x|>\delta \\
V_{\delta}^{\prime}(x)= \begin{cases}q_{\delta}, & \text { for } 1 \leq x, \\
q_{\delta}^{\prime}, & \text { for } x \leq-1\end{cases}
\end{array}\right.
$$

with some $q_{\delta}\left(=q_{\delta}^{M}\right)$ and $q_{\delta}^{\prime}\left(=q_{\delta}^{\prime M}\right) \notin P$.

(ii) Assume condition (F1'). For each $S \in(0, T)$ there exists (large) $B_{\delta}(=$ $\left.B_{\delta}^{M, S}\right) \geq 0$ such that $V_{\delta}^{+} \in A_{P}\left(Q_{s}\right)$ is a supersolution of (E) in $Q_{s}=$ $(0, S) \times \mathbf{R}$ of the form

$$
V_{\delta}^{+}(t, x)\left(=\left.V_{\delta}^{+, M, s}\right|_{Q_{s}}\right)=B t+V_{\delta}(x) \quad \text { for }(t, x) \in \overline{Q_{S}} \quad \text { and } B \geq B_{\delta} .
$$

Here we suppress the dependence of $M$ and $S$ in $V_{\delta}^{+}$and $B_{\delta}$.

Proof. It is clear that Lemmas 9.3 and 9.4 with $\Omega_{1}=\mathbf{R}, \Omega_{2}=(-1,1)$ and $\Omega_{3}=(-1 / 2,1 / 2)$ yield the result of (i). Now we give the proof of (ii). We

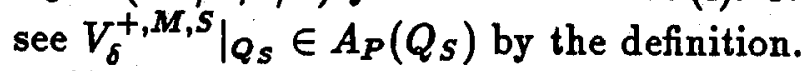

Since the number of faceted regions of $V_{\delta}$ is finite, we have

$$
c_{1}=\sup \left\{\left|\Lambda_{W}\left(V_{\delta}, \boldsymbol{x}\right)\right| ; \boldsymbol{x} \in \mathbf{R}, V_{\delta}^{\prime}(\boldsymbol{x}) \in P\right\}<\infty \text {. }
$$

We also have

$$
c_{2}=\sup \left\{\left|\Lambda_{W}\left(V_{\delta}, x\right)\right| ; x \in \mathbf{R}, V_{\delta}^{\prime}(x) \notin P\right\}<\infty,
$$


since

$$
\sup \left\{\left|V_{\delta}^{\prime \prime}(x)\right| ;|x| \leq 1\right\}<\infty
$$

and

$$
\sup \left\{\left|W^{\prime \prime}(p)\right| ; q_{\delta} \leq p \leq q_{\delta}^{\prime}, p \notin P\right\}<\infty
$$

by the assumption of $W$. Thus we observe that

$$
\sup \left\{\left|\Lambda_{W}\left(V_{\delta}, x\right)\right| ; x \in \mathbf{R}\right\}<c_{3}<\infty
$$

with $c_{3}=\max \left(c_{1}, c_{2}\right)$.

Since condition (F1') yields

$$
c_{4}=\inf \left\{F(t, p, X) ; t \in[0, S], p \in\left[q_{\delta}, q_{\delta}^{\prime}\right],|X| \leq c_{3}\right\}>-\infty
$$

we have

$$
F\left(t, V_{\delta}^{\prime}(x), \Lambda_{W}\left(V_{\delta}, x\right)\right) \geq c_{4} \quad \text { for all }(t, x) \in \overline{Q_{s}} .
$$

We take $B_{\delta}$ so that $B_{\delta} \geq \max \left(-c_{4}, 0\right)$. Function $V_{\delta}^{+}$is a supersolution of (E) in $Q_{s}$. In fact, if $((\hat{t}, \hat{x}), \psi) \in Q_{s} \times A_{P}\left(Q_{s}\right)$ satisfies

$$
\min _{Q_{s}}\left(V_{\delta}^{+}-\psi\right)=\left(V_{\delta}^{+}-\psi\right)(\hat{t}, \hat{x})
$$

it holds that

$$
\begin{aligned}
\psi_{t}(\hat{t}, \hat{x})+F\left(\hat{t}, \psi_{x}(\hat{t}, \hat{x})\right), \Lambda_{W}(\psi(\hat{t}, \cdot), \hat{x}) & =B+F\left(\hat{t}, V_{\delta}^{+}(\hat{x}), \Lambda_{W}\left(V_{\delta}^{+}, \hat{x}\right)\right) \\
& \geq B-B_{\delta} \geq 0
\end{aligned}
$$

since $V_{\delta} \in C_{P}^{2}(\mathbf{R})$. It is clear that $\left(\left.V_{\delta}\right|_{Q_{s}}\right)_{*} \geq 0 \geq-\infty$ in $[0, S) \times \mathbf{R}$.

9.6. Lemma. Suppose that $u_{0}$ is bounded and uniformly continuous in $\mathbf{R}$, so that for each $\varepsilon \in(0,1)$ there exists $\delta=\delta(\varepsilon) \in(0,1 / 2)$ satisfying

$$
\left|u_{0}(x)-u_{0}(\xi)\right|<\varepsilon \quad \text { for }|x-\xi|<\delta .
$$

Let $V_{\delta(e)} \in C_{P}^{2}(\mathbf{R})$ be as in Lemma 9.5 (i) with $M=\max _{x \in R} u_{0}(x)-$ $\min _{x \in \mathbf{R}} u_{0}(x)$. Then we have

$$
u_{0}(x)=\inf \left\{V_{\delta(\varepsilon)}(x-\xi)+u_{0}(\xi)+\varepsilon ; \varepsilon \in(0,1), \xi \in \mathbf{R}\right\}
$$

Proof. Since (9.4) implies

$$
u_{0}(x)<u_{0}(\xi)+\varepsilon \quad \text { for }|x-\xi| \leq \delta(\varepsilon)
$$


and the definition of $M$ implies

$$
u_{0}(x)<u_{0}(\xi)+M \leq u_{0}(\xi)+V_{\delta(\varepsilon)}(x-\xi) \quad \text { for }|x-\xi|>\delta(\varepsilon),
$$

we have

$$
u_{0}(x) \leq V_{\delta(\varepsilon)}(x-\xi)+u_{0}(\xi)+\varepsilon \quad \text { for } x, \xi \in \mathbf{R} \text { and } \varepsilon \in(0,1)
$$

For each $\boldsymbol{x} \in \mathbf{R}$ and $\varepsilon^{\prime}>0$, we have

$$
V_{\delta(\varepsilon)}(x-\xi)+u_{0}(\xi)+\varepsilon<\varepsilon^{\prime}+u_{0}(x)
$$

with $\xi=x$ and $\varepsilon=\varepsilon^{\prime} / 2$, which yields the results.

Proof of Lemma 9.1. Firstly, for each $\varepsilon \in(0,1)$ let $V_{\delta(e)} \in C_{P}^{2}(\mathbf{R})$ be the same as Lemma 9.6. Secondly, for each $\varepsilon \in(0,1)$ and $\xi \in \mathbf{R}$ we set

$$
u^{+, \varepsilon}(t, x ; \xi)=V_{\delta(\varepsilon)}^{+}(t, x-\xi)+u_{0}(\xi)+\varepsilon \quad \text { for }(t, x) \in \overline{Q_{S}}
$$

with $B=B_{\delta(e)}$. Then $u^{+, e}(t, x ; \xi)$ belongs to $A_{P}\left(Q_{S}\right)$ and is a supersolution of (E) in $Q_{s}$ by Lemma 9.5 (ii). Lastly, we take

$$
u^{+}(t, x)=\inf \left\{u^{+, e}(t, x, \xi) ; \varepsilon \in(0,1), \xi \in \mathbf{R}\right\} \quad \text { for }(t, x) \in \overline{Q_{s}}
$$

then $u^{+}$is uppersemicontinuous in $\overline{Q_{s}}$ and

$$
\left(\left.u^{+}\right|_{Q_{s}}\right)_{*} \geq \min _{\xi \in \mathbb{R}} u_{0}(\xi)>-\infty \quad \text { for }[0, S) \times \mathbf{R} .
$$

So, Lemma 8.1' yields that $u^{+}$is a supersolution of (E) in $Q_{s}$. Now we have (9.2). In fact, Lemma 9.6 implies

$$
u^{+}(0, x)=u_{0}(x) \quad \text { for } x \in \mathbf{R}
$$

and

$$
u^{+}(t, x) \geq u_{0}(x) \quad \text { for }(t, x) \in \overline{Q_{s}},
$$

since $B_{\delta(\varepsilon)} \geq 0$ and

$$
V_{\delta(\bullet)}^{+}(t, x-\xi) \geq V_{\delta(\varepsilon)}(x-\xi) \quad \text { for }(t, x) \in \overline{Q_{s}} \text { and } \xi \in \mathbf{R} .
$$

We can get the lowersemicontinuous function $u^{-}$satisfying the desired property parallelly 
Proof of Remark 9.2. If $u_{0}$ is periodic with period $\varpi$, function $u^{+}$constructed in the proof of Lemma 9.1 satisfies (9.3), since

$$
\begin{aligned}
u^{+, \iota}(t, x+\varpi ; \xi) & =V_{\delta(\iota)}^{+}(t, x+\varpi-\xi)+u_{0}(\xi)+\varepsilon \\
& =V_{\delta(\iota)}^{+}(t, x-(\xi-\varpi))+u_{0}(\xi-\varpi)+\varepsilon \\
& =u^{+, \iota}(t, x, \xi-\varpi) .
\end{aligned}
$$

\section{Proof of Theorem 3.5.}

Step1 (Existence on $Q_{S}$ ). Since (F1) implies (F1') for each $S \in(0, T)$, Lemma 9.1 is applicable. For each $S \in(0, T)$, let $u^{+}$, and $u^{-}$, be an upper-, and a lowersemicontinuous function in $Q_{S}$ obtained in Lemma 9.1, respectively. By Theorem 3.4, there exists a generalized solution $\tilde{u}$ of $(\mathrm{E})$ in $Q_{s}$ such that

$$
u^{-} \leq \tilde{u} \leq u^{+} \quad \text { in } Q_{S}
$$

and

$$
\tilde{u}(t, x+\varpi)=\tilde{u}(t, x) \quad \text { for }(t, x) \in Q_{S} .
$$
have

Since $u^{+}$(resp. $u^{-}$) is upper- (resp. lower-) semicontinuous in $\overline{Q_{s}}$, we

$$
-\infty<u^{-} \leq \tilde{u}_{*} \leq \tilde{u}^{*} \leq u^{+}<\infty \quad \text { in } \overline{Q_{s}} .
$$

Since $u^{-}(0, x)=u^{+}(0, x)=u_{0}(x)$ in $\mathbf{R}$, we have

$$
\tilde{u}_{m}(0, x)=\tilde{u}^{*}(0, x)=u_{0}(x) \quad \text { in } \mathbf{R} \text {. }
$$

Theorem 3.2 yields

$$
\tilde{u}^{*} \leq \tilde{u}_{*} \quad \text { in } Q_{s}, \quad \text { that is, in } \overline{Q_{S}}
$$

which implies that $\tilde{u}^{*}$ is continuous in $\overline{Q_{S}}$. We set $u^{S}=\tilde{u}^{*}$ in $[0, S)$. Then $u^{S}$ is a generalized solution of (E) in $Q_{S}$ and $u^{S} \in C([0, S) \times \mathbf{R})$ satisfies

$$
\begin{array}{ll}
u^{S}(0, x)=u_{0}(x) & \text { for } x \in \mathbf{R} \\
u^{S}(t, x+\varpi)=u^{S}(t, x) & \text { for }(t, x) \in[0, S) \times \mathbf{R}
\end{array}
$$

Step2 (Uniqueness on $Q_{s}$ ). Let $v^{S} \in C([0, S) \times \mathbf{R})$ satisfy (9.5) replacing $u^{S}$ to $v^{s}$, such that $v^{s}$ is a generalized solution of $(\mathrm{E})$ in $Q_{s}$. Theorem 3.2 yields that $u^{S}=v^{S}$ in $[0, S) \times \mathbf{R}$, which implies the uniqueness of $u^{S}$ in $[0, S) \times \mathbf{R}$.

Step3 (Unique existence on $(0, T) \times \mathbf{R}$ ). If $0<S<S^{\prime}<T$, Proposition 6.19 implies that $\left.u^{S^{\prime}}\right|_{Q_{S}}$ is a generalized solution of $(\mathrm{E})$ in $Q_{s}$. By Step 2 
for $t \in(0, T)$, it is possible to define $u$ from $\left\{u^{S} ; 0<S<T\right\}$ uniquely as follows:

$$
u(t, x)=u^{S}(t, x) \quad \text { with } S \in(t, T) .
$$

From Proposition 6.20 we see that $u$ is a generalized solution of $(E)$ in $(0, T) \times$ R. It is easy to see that other properties hold.

\section{ACKNOWLEDGEMENTS}

We are grateful to Professor Masaru Suzuki and Professor Makio Uwaha for valuable suggestions related to crystal growth of Helium. This work was initiated while the authors visited the Johns Hopkins University in 1994. Their hospitality and support are gratefully acknowledged as well as support from Japanese Society of Promotion of Sciences. Support to the second author from THE SUHARA FOUNDATION and a Grant-in-Aid for Scientific Research (07044094), International Science Research Program, Joint Research, the Ministry of Education, Science and Culture is gratefully acknowledged. Additional support the Special Grant-in-Aid for Promotion of Education and Science in Hokkaido University provided by the Ministry of Education, Science and Culture is acknowledged.

\section{REFERENCES}

[AT] F. Almgren and J. Taylor, Flat flow is motion by crystalline curvature for curves with crystalline energies, preprint, University of Minnesota, Geometry Center.

[ATW] F. Almgren, J. Taylor and L. Wang, Curvature driven flows: $A$ variational approach, SIAM J. Control Optim. 31 (1993), 387437.

[AG1] S. B. Angenent and M. E. Gurtin, Multiphase thermomechanics with interfacial structure 2., Evolution of an isothermal interface, Arch. Rational Mech. Anal. 108 (1989), 323-391.

[AG2] S. B. Angenent and M. E. Gurtin, Anisotropic motion of a phase interface, J. reine angew. Math. 446 (1994), 1-47.

[CGG] Y. -G. Chen, Y. Giga and S. Goto, Uniqueness and existence of viscosity solutions of generalized mean curvature flow equations, J. Differential Geom. 33 (1991), 749-786.

[Ch] A. A. Chernov, Modern Crystallography III, Crystal Growth, Solid-State Sciences 36, Springer Verlag 1984, (especially §3.3).

[CEL] M. G. Crandall, L. C. Evans and P. L. Lions, Some properties of viscosity solutions of Hamilton-Jacobi equations, Trans. Amer. Math. Soc. 282 (1984), 487-502. 
[CIL] M. G. Crandall, H. Ishii and P. L. Lions, User's guide to viscosity solutions of second order partial differential equations, Bull. Amer. Math. Soc. 27 (1992), 1-67.

[EGS] C. M. Elliott, A. R. Gardiner and R. Schätzle, Crystalline curvature flow of a graph in a variational setting, Univ. of Sussex at Brighton, Centre for mathematical analysis and its applications, Research report No.95/06.

[ES] L. C. Evans and J. Spruck, Motion of level sets by mean curvature. I, J. Differential Geom. 33 (1991), 635-681.

[FK] I. Fonceca and M. Katsulakis, $\Gamma$-convergence, minimizing movements and generalized mean curvature evolution, Differential and Integral Equations 8 (1995), 1619-1656.

[FG] T. Fukui and Y. Giga, Motion of a graph by nonsmooth weighted curvature, Proc. First World Congress of Nonlinear Analysts, (ed. V. Lakshmikantham) Walter de Gruyter, Berlin, vol.I(1995), 4756 I (1995), 47-56.

[GMHG1] M.-H. Giga and Y. Giga, Geometric evolution by nonsmooth interfacial energy, Banach Center Pub., to appear.

[GMHG2] M.-H. Giga and Y. Giga, Consistency in evolutions by crystalline curvature, Proc. Free Boundary Problems '95, Theory and Applications (ed. M. Niezgódka), Zakopane, Poland(1995), to appear.

[G] Y. Giga, Motion of a graph by convexified energy, Hokkaido Math. J. 23 (1994), 185-212.

[GGo] Y. Giga and S. Goto, Motion of hypersurfaces and geometric equations, J. Math. Soc. Japan 44 (1992), 99-111.

[GGIS] Y. Giga, S. Goto, H. Ishii and M.-H. Sato, Comparison principle and convexity preserving properties for singular degenerate parabolic equations on unbounded domains, Indiana Univ. Math. J. 40 (1991), 443-470.

[GGu] Y. Giga and M. E. Gurtin, A comparison theorem for crystalline evolution in the plane, Quart. J. Appl. Math., to appear.

[GGuM] Y. Giga, M. E. Gurtin and J. Matias, On the dynamics of crystalline motions, preprint.

[Gir] P. M. Girão, Convergence of a crystalline algorithm for the motion of a simple closed convex by weighted curvature, SIAM J. Numer. Anal. 32 (1995), 886-899.

[GirK1] P. M. Girão and R. V. Kohn, Convergence of a crystalline algorithm for the heat equation in one dimension and for the motion of a graph by weighted curvature, Numer. Math. 67 (1994), 41-70.

[GirK2] P. M. Girão and R. V.Kohn, The crystalline algorithm for computing motion by curvature, Variational Methods for Discontinuous Structure, (eds. R. Serapioni and F. Tomarelli) Birkhauser, 
to appear.

[Go] S. Goto, Generalized motion of hypersurfaces with superlinear growth speed in curvature tensor, Differential and Integral Equations 7 (1994), 323-343.

[Gu] M. E. Gurtin, Thermomechanics of Evolving Phase Boundaries in the Plane, Oxford, Clarendon Press, 1993.

[GSS] M. E. Gurtin, H. M. Soner and P. E. Souganidis, Anisotropic motion of an interface relaxed by formulation of infinitesimal wrinkles, J. Differential Equations 119 (1995), 54-108.

[I] H. Ishii, Perron's method for Hamilton-Jacobi equations, Duke Math. J. 55 (1987), 369-384.

[IS] H. Ishii and P. Souganidis, Generalized motion of noncompact hypersurfaces with velocity having arbitrary growth on the curvature tensor, Tohoku Math. J. 47 (1995), 227-250.

[IR] H. Ishii and M. Ramaswamy, Uniqueness results for a class of Hamilton-Jacobi equations with singular coefficients, Commun. in Partial Differential Equations 20 (1995), 2187-2213.

[KK] B. Kawohl and N. Kutev, Global behaviour of solutions to a parabolic mean curvature equation, Differential and Integral Equations 8 (1995), 1923-1946.

[Ko] Y. Kōmura, Nonlinear semi-groups in Hilbert space, J. Math. Soc. Japan 19 (1967), 493-507.

[LL] J. M. Lasry and P. L. Lions, $A$ remark on regularization in Hilbert spaces, Israel J. Math. 55 (1986), 257-266.

[LSU] O. A. Ladyzhenskaya, V. Solonnikov and N. Ural'ceva, Linear and Quasilinear Equations of Parabolic Type, Translations of Mathematical Monographs, Amer. Math. Soc. 23 (1968).

[LS] S. Luckhaus and T. Sturzenhecker, Implicit time discretization for the mean curvature flow equations, Calculus of Variations and Partial Differential Equations 3 (1995), 253-271.

[MBK] H. Müller-Krumbhaar, T. W. Burkhardt and D. M. Kroll, $A$ generalized kinetic equation for crystal growth, J. Crystal Growth 38 (1977), 13-22.

[OKS] M. Ohnuma and K. Sato, Singular degenerate parabolic equations with applications to the $p$ - Laplace diffusion equation, preprint.

[OhS] M. Ohnuma and M. -H. Sato, Singular degenerate parabolic equations with applications to geometric evolutions, Differential and Integral Equations 6 (1993), 1265-1280.

[R] A. R. Roosen, Crystalline curvature and flat flow in a diffusion field, preprint (1994).

[St] A. Stancu, Uniqueness of self-similar solutions for a crystalline flow, preprint. 
[T1] J. Taylor, Constructions and conjectures in crystalline nondifferential geometry, Proceedings of the Conference on Differential Geometry, Rio de Janeiro, pp.321-336, Pitman, London, 1988.

[T2] J. Taylor, Mean curvature and weighted mean curvature, Acta Metall. 40 (1992), 1475-1485.

[T3] J. Taylor, Motion of curves by crystalline curvature, including triple junctions and boundary points, In: Differential Geometry: Partial Differential Equations on Manifolds (eds. R. Greene and S. T. Yau), Proc. Symp. Pure Math., 54(1993) Part I, pp. 417-438, Amer. Math. Society Providence, RI.

[TCH] J. Taylor, J. Cahn and C. Handwerker, Geometric models of crystal growth, Acta Metall. 40 (1992), 1443-1474.

[Y] K. Yosida, Functional Analysis, 6-th edition, Springer-Verlag, Berlin, Heidelberg, New York, 1980. 